\title{
Autonomous Cooperative Flight of Rigidly Attached Quadcopters
}

\author{
DIEGO GONZÁLEZ MORÍN
}




\title{
Autonomous Cooperative Flight of Rigidly Attached Quadcopters
}

\author{
DIEGO GONZÁLEZ MORÍN
}

Master Program in Robotics, Systems and Control Date: June 14, 2018

Supervisor: Soma Tayamon, José Araujo and Jaehyun Yoo Examiner: Karl Henrik Johansson

Swedish title: Autonom kooperativ flygning av stelt sammankopplade quadcopters

School of Electric Engineering 



\section{Abstract}

Abstract - Research within the area of Unmanned Aerial Vehicles (UAVs) is a continuously expanding field due to their wide and diverse range of applications such as inspection, monitoring, mapping, precision agriculture, aerial imaging and entertainment. However, their application in tasks such as transportation, tool manipulation or assistance in emergency situations still poses many challenges.

In this thesis, a novel strategy for cooperative flight of physically attached quadcopters which allows the quadcopters to perform the transportation of heavier loads and carrying out more complex tasks is proposed. Specifically, a method for online and automatic modelling and control of the quadcopters without human intervention is introduced.

First, the method performs the estimation of the physical structure attaching the quadcopter's solely relying on information from the quadcopters Inertial Measurement Unit (IMU) obtained via simple and short online experiments. Then, given the estimated physical structure, a stable operation of the quadcopters is achieved via a distributed controller, where the controller parameters are obtained via reinforcement learning. Finally, experimental results validate the proposed method, showing that a correct estimation of the physical structure is obtained as well as a stable flight is achieved for a set of connected quadcopters. 


\section{Sammanfattning}

Forskning inom obemannade luftfarkoster (UAVs) är ett kontinuerligt expanderande område på grund av dess breda omfång inom en rad olika tillämpningsområden, däribland inspektion, övervakning, mappning, jordbruksprecision, luftavbildning och underhållning. Likväl finns det fortfarande flertalet utmaningar inom applikationer så som transportering, manipulering av verktyg eller assistans vid nödsituationer.

I den här avhandlingen föreslås en ny strategi för kooperativ flygning av fysiskt sammankopplade quadcopters som tillåter dessa att transportera tyngre laster och genomföra mer komplexa uppgifter. Specifikt, en metod för uppkopplad och automatisk modellering och reglering av quadcopters utan mänskligt ingripande föreslås.

Metoden startar med att genomföra estimering av den fysiska strukturen som sammankopplar quadcoptrarna enbart genom att förlita sig på information från quadcoptrarnas tröghetsmätningsenhet (IMU) erhållna via enkla och korta uppkopplade experiment. Givet den estimerade fysiska strukturen, uppnås därefter en stabil operation av quadcoptrarna via en distribuerad kontroller, vars kontrollparametrar erhålls via förstärkande inlärning. Slutligen valideras den föreslagna metoden genom experimentella resultat som visar att en korrekt estimering av den fysiska strukturen är erhållen tillika en uppnådd stabil flygning för en uppsättning av sammankopplade quadcopters. 


\section{Acknowledgments}

Primero me gustaria dar las gracias a mis padres, ellos son los que 1levan dos años no solo haciendo un esfuerzo económico enorme, pero además decir adiós a un hijo siempre es duro. Lo se porque también ha sido duro decirles adios a ellos. Han sabido estar no estando, apoyarme, darme cualquier cosa que necesitase. Han sufrido conmigo a lo largo de estos dos años, y por supuesto, han celebrado mis pequeños logros. Gracias a ellos, por esas miradas de orgullo aún cuando no entendian absolutamente nada de lo que estaba haciendo. Gracias, porque fuisteis y seguís siendo el molde, la guía y la brújula y soy quien soy por vosotros.

Cristina, gracias por haber seguido luchando cada dia sin ninguna duda. Has sido mi primer apoyo en todo momento. La primera persona con la que celebraba lo bueno y a la primera a la que le contaba lo malo. Eres una de las principales razones por las que estoy donde estoy. Durante todo este tiempo no has parado de mirarme orgullosa de mi, y me has dado fuerzas en los peores días en los que todo se veía muy negro. Gracias, gracias, gracias.

Soma and José, thank you very much for placing your trust in me after that first Skype interview. You both were two amazing supervisors, you gave total freedom to implement my ideas and you have patience with all my "this is super easy" that turned to be several weeks of not that easy work. This Master Thesis can be readable only because you read and correct it over more than 7 iterations. Thank you for keep placing your trust in me now.

Gracias a Roberto y Mario. Se que la distancia ha hecho mella, pero quiero agradeceros que sigais ahí. Agradeceros tanto las conversaciones serias como las más estúpidas de todas. La distancia seguira mellando, pero se que no faltareis.

Thank you Javi, Sisi and Nikola for all the time that we spent together during the last months. For all the dinners after a long day in the office, the movie nights and nights out. Thank you for being my main support here in Sweden.

Guille, gracias por todas las veces que he podido recargar las pilas a tope cuando volvía a casa a visitaros. Siempre aprendo algo de ti que me hace ser mejor.

Gracias abuelo. Siempre intento hacer las cosas pensando en si te harían sentir orgulloso. 


\section{Contents}

1 Introduction 1

1.1 Motivation . . . . . . . . . . . . . . . . . . 4

1.2 Research Question . . . . . . . . . . . . . . . . 5

1.3 Literature Review . . . . . . . . . . . . . . . . . . . . . 5

1.4 Scope $\ldots \ldots \ldots \ldots \ldots \ldots$

1.5 Outline $\ldots \ldots \ldots \ldots \ldots$

$\begin{array}{lll}2 & \text { Preliminaries } & 10\end{array}$

2.1 Notation . . . . . . . . . . . . . . . . . . . 10

2.2 Quadcopter Modeling . . . . . . . . . . . . . . . . . 10

2.2.1 Coordinate Frame and Rotation Matrix . . . . . 11

2.2 .2 Force Equations . . . . . . . . . . . . . . . . . 13

2.2 .3 Momentum Equations . . . . . . . . . . . . . . . 14

2.2.4 Integration of the Dynamical Model Equations . . 17

2.3 PID Controller . . . . . . . . . . . . . . . . . . . . 18

2.4 Neural Networks . . . . . . . . . . . . . . . . . . . . . 19

2.5 Reinforcement Learning . . . . . . . . . . . . . . . . . . 21

2.5 .1 Deep Q-Learning . . . . . . . . . . . . . . . . 22

2.6 Kalman Filter . . . . . . . . . . . . . . . . . . . . . . 23

3 Dynamical Model of Rigidly Attached Quadcopters 25

3.1 Force Equations . . . . . . . . . . . . . . . . . 26

3.2 Momentum Equations … . . . . . . . . . . . 27

3.3 Inertia Matrix $\ldots \ldots \ldots \ldots \ldots . \ldots \ldots$

3.4 Integration of the Dynamical Model Equations . . . . . . 30

3.5 Noise and Bias . . . . . . . . . . . . . . . . . . 30

4 Estimation of the Physical Connection Parameters of Rigidly Attached Quadcopters $\quad 32$

$4.1 \quad$ Data Acquisition and Preprocessing Strategy . . . . . . . 34 
4.1 .1 Dynamic Oscillations . . . . . . . . . . . . . . 34

4.2 Data Filtering . . . . . . . . . . . . . . . 35

4.2 .1 Data Interpolation . . . . . . . . . . . . . . . . . . . . . . . . . . .

4.3 Parameter Estimation . . . . . . . . . . . . . . . . . . 41

4.3 .1 Relative z-axis Rotation . . . . . . . . . . . . . 43

4.3 .2 Distances. . . . . . . . . . . . . . 44

4.3 .3 Absolute z-axis Rotation Within Each Pair . . . . 53

4.3.4 Absolute Relative Rotations and Positions . . . . 55

4.3 .5 Final Overview . . . . . . . . . . . . . 56

4.4 Localization Approach . . . . . . . . . . . . 57

5 Controller Design for Rigidly Attached Quadcopters 60

5.1 Control Architecture and Strategy . . . . . . . . . . 60

5.1 .1 Control Architecture . . . . . . . . . . . . . . . 60 60

5.1 .2 Controller Design . . . . . . . . . . . . . . . . 62

5.2 Controller Design . . . . . . . . . . . . . . . . . . . . . . . . 63

5.3 Controller Tuning . . . . . . . . . . . . . . . . . 65

5.3.1 Reinforcement Learning PID Tuning: Deep QLearning .................. 66

\begin{tabular}{|ll}
5.4 & Adaptive Control: PID Gains Interpolation Using Neu- \\
\hline & ral Networks $\ldots \ldots \ldots \ldots$
\end{tabular}

$\begin{array}{lll}6 & \text { Experimental Setup } & \mathbf{7 6}\end{array}$

6.1 Hardware ................... . . 76

6.1 .1 Crazyflie . . . . . . . . . . . . . . . 76

6.1 .2 Rigid Attachment . . . . . . . . . . . . . . . . . . . . . . 79

6.2 Global Positioning . . . . . . . . . . . . . . . . . . 81

6.2 .1 Motion Capture System . . . . . . . . . . . . . . . 82

6.2.2 Loco Positioning System . . . . . . . . . . . . . . . 83

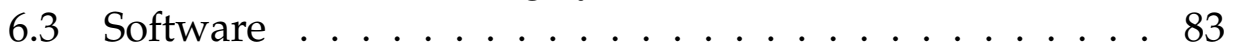

6.3.1 Simulator and User Interface Application . . . . . 83

6.3 .2 Crazyflie's Firmware Setup . . . . . . . . . . . . 89

6.4 Architecture overview . . . . . . . . . . . . . . 91

\begin{tabular}{lll}
\hline 7 & Results & 93
\end{tabular}

$7.1 \quad$ Description of Experiments . . . . . . . . . . . . . . . . 93

7.1 .1 Parameter Estimation . . . . . . . . . . . . . 94

7.1 .2 Control Architecture and Strategy . . . . . . . . . 95

7.1 .3 Full approach . . . . . . . . . . . . . . . . . 97

7.2 Simulation Results . . . . . . . . . . . . . . . . . . . 98 
$7.2 .1 \quad$ Parameter Estimation . . . . . . . . . . . . . . . 98

7.2 .2 Control Architecture and Strategy . . . . . . . . . 100

7.3 Experimental Results . . . . . . . . . . . . . . . . . . . 102

7.3.1 Parameter Estimation Experiments. . . . . . . . . 102

7.3.2 Control Architecture and Strategy . . . . . . . . 103

\begin{tabular}{|ll}
\hline 7.3 .3 & Full Approach: Parameter Estimation and Con- \\
\hline & trol Architecture $\ldots \ldots \ldots \ldots \ldots \ldots \ldots \ldots \ldots$
\end{tabular}

8 Conclusions and Future Work 116

8.1 Conclusion . . . . . . . . . . . . . . . . . . . . . 116

8.2 Future Work . . . . . . . . . . . . . . . . . . . . . 117 


\section{Chapter 1}

\section{Introduction}

The fast development of technology in the last century was triggered by an increased and improved automation. Automation was born as a tool in the industry to increase the efficiency in many processes and the production volume while decreasing the costs. Nowadays, automation is included in most of the everyday use devices and technologies, such as smartphones, commercial aircrafts and self-driven cars. It is currently not only limited to the completion of repetitive tasks, but also decision making, pattern recognition and social interaction.

Within the field of robotics, the interest in autonomous vehicles has increased over the last decade. The race for the autonomous car has triggered a significant increment in the investment of different technological fields related to automation: sensors, actuators, processors, etc. With this increment, autonomous vehicles became a large field of research.

Autonomous vehicles can be divided in four main groups: Unmanned Ground Vehicles (UGVs) [22], Autonomous Underwater Vehicles (AUVs) [76], Autonomous Surface Vehicles (ASVs) [40] and Unmanned Aerial Vehicles (UAVs) [27]. Unmanned Ground vehicles can also be subdivided depending on the main actuation system that they use. The options are multiple, from wheels, to legs or even crawling robot (See Fig. 1.1). The chosen actuators will depend on the type of terrain that the vehicle is designed for. Their lack of terrain versatility is considered their main disadvantage. However, with the current technology, they are able to deal with high payloads ensuring a long autonomy.

Most Unmanned Underwater Vehicles design is based on the canonic 


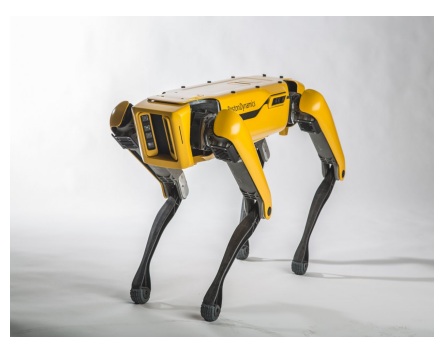

(a)

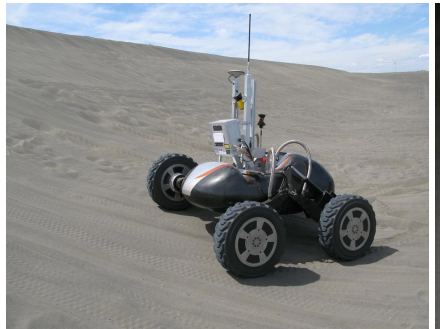

(b)

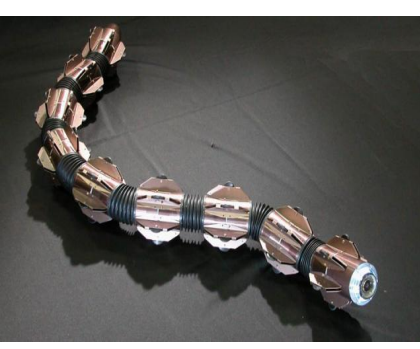

(c)

Figure 1.1: (a) Boston Dynamics SpotMini [17]. (b) NASA's Lunar Autonomous Rover SCARAB [56]. (c) ACM-R5H Amphibious Snake Robot by HiBot [29].

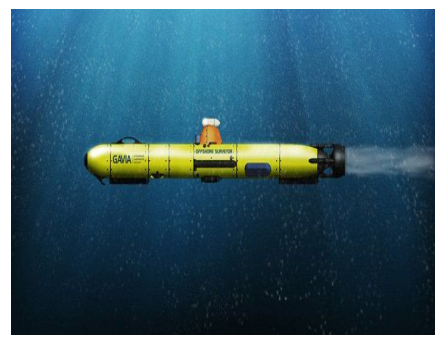

(a)

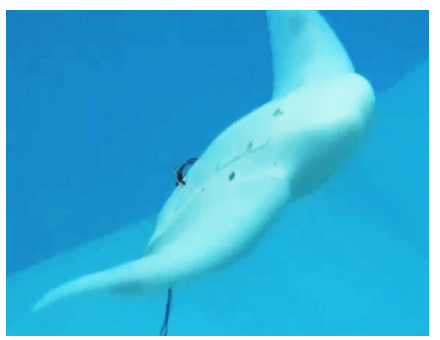

(b)

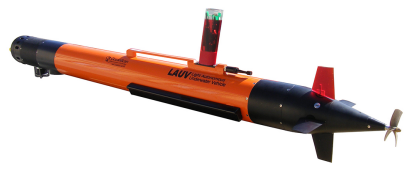

(c)

Figure 1.2: (a) GAVIA AUV developed by the Russian military department [48]. (b) Mantbot designed by Virginia University [12]. (c) Light Autonomous Underwater Vehicle LAUV by LSTS [43]

submarines. However, there are some examples of biologically based designs, like the Mantabot, developed in the University of Virginia (see Fig. 1.2). These group of Unmanned Vehicles are usually used for underwater exploration, mine deactivation and mapping.

Autonomous Surface Vehicles are vehicles that operate on the surface of the water. They are used for a wide range of military and commercial applications such as: Mine Counter Measures (MCM), surveillance, oceanographic data collection and underwater construction. Some examples of this group of autonomous vehicles are shown in Fig. 1.3 .

Finally, the use of UAVs has spread not only within the research or military fields, but also in the commercial area. From small toys for kids, to robust commercial UAVs used in a wide variety of tasks. The interest in UAVs is growing quickly and it is a recurrent topic in research. UAVs can be classified in 3 big groups depending on their morphology: fixed wing, multirotor and Vertical Take Off and Landing 


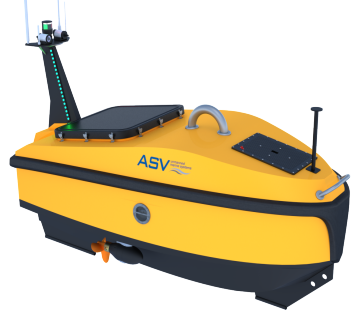

(a)

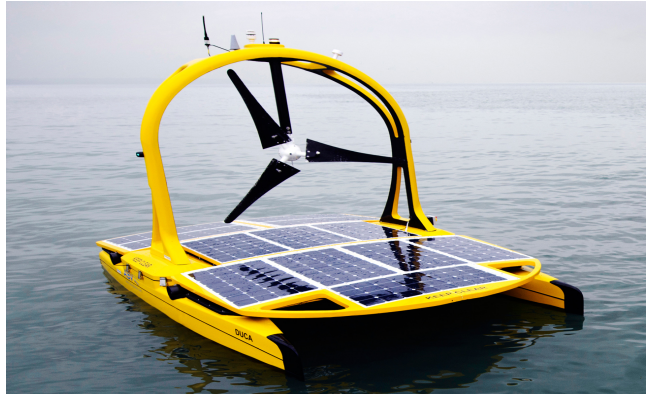

(b)

Figure 1.3: (a) C-Stat Station Keeping Buoy by ASV unmanned marine systems [72]. (b) C-Enduro USV by ASV unmanned marine systems [71].

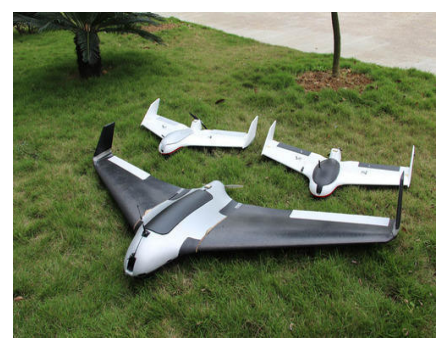

(a)

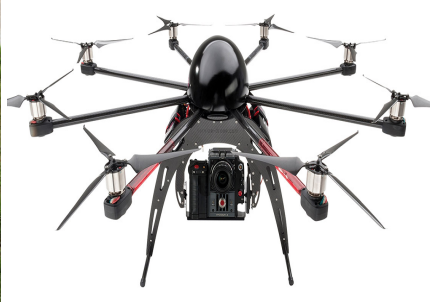

(b)

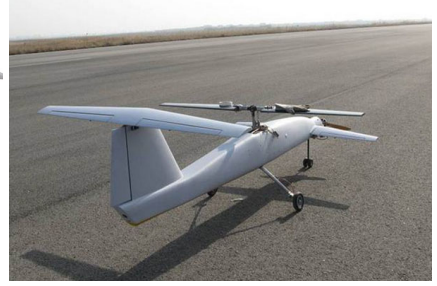

(c)

Figure 1.4: (a) Skywalker X8 fixed wing UAV by TechnoSys [69]. (b) Multirotor G4 Skycrane UAV by Multirotor [54]. (c) MH-VTOL-2 Vertical Take-off and Landing Unmanned Aerial Vehicle from Mh Elec [18].

(VTOLs). Examples of this morphologies are shown in Fig. 1.4.

The current thesis will focus on the multirotor UAVs. The morphologies, sizes and characteristics are wide, from micro-UAVs with only 4 motors, like the Crazyflie quadcopter [6], to huge multirotors like the Volocopter, with the capacity of human transportation. The variety of applications has spread over the last decade: structures inspection, agriculture, mapping, military purposes and the entertainment business.

One of the main limitations of the possible applications is the maximum payload and flight time, both qualities are very related. For many of the proposed examples of applications, payload transportation is required. Payload transportation has 2 main consequences: 
first, a worse dynamical response of the system and second, a shorter flight time. In addition, the maximum payload is different in each quadcopter and it constraints the possible applications of each model.

\subsection{Motivation}

The balance of maximum payload and flight time is a very sensitive constraint when studying the possible applications of a set of quadcopters. Several research lines proposed possible solutions for this matter. A popular solution is to use several quadcopters collaborating to transport the load [35][59][63][58]. This way, the load lifted by each quadcopter is reduced, increasing the flight time of the system. Furthermore, collaborative aerial transportation allows to carry heavier loads.

The objective of the current thesis is to explore this idea of collaborative aerial transportation, proposing a different strategy and approach. Most of the collaborative aerial transportation solutions considered that the attachment between the load and the quadcopters is not rigid, allowing the quadcopters in the system to move independently. However, for some applications, the connection between the quadcopters must be rigid, example given: a set of quadcopters used to carry a big video camera needs a rigid attachment to ensure enough stability of the image. On the contrary, the rigid attachment solution has not been deeply researched.

Multiple quadrotors can be attached to each other using rigid connections. In a practical scenario, these quadcopters could connect to each other automatically, similarly to [63], or with human help. However, the attachment properties, such as the length or how they are connected, may not be known a priori at all or not with sufficient precision.

As they are physically attached, their controllers must be redesigned to accommodate these changes in the dynamics of the system. This brings the need of developing a solution to estimate the connection parameters that are not known a priori. 


\subsection{Research Question}

As expressed in the title, the principal research question of the current thesis is to design an approach to achieve autonomous cooperative flights of rigidly attached quadcopters. This research question encompasses the following questions as well:

- How to estimate the attachment parameters of the set of rigidly attached quadcopters?

- How to efficiently design a controller architecture to ensure system stability?

\subsection{Literature Review}

Aerial collaboration has lately become a very recurrent research topic. However, the research path can be divided in two big blocks: nonrigid attachments and rigid attachments. Within the first group, the most common approach is the use of cable-based attachments. This approach allow independent control of the agents, constituting one of the main challenges, the obstacle avoidance between the quadcopters, while ensuring stability of the transported load [23][52][51].

More interesting for the development of the current thesis are the examples of rigid attachment based collaborative aerial control. In [38], they proposed a control method for the case of two quadcopters rigidly attached to a bar, see Fig. 1.5(a). The control architecture is based on a proportional-derivative controller that incorporates the equations of the dynamics of the system to adapt the controller architecture to every possible configuration. This is achieved by including the expression of the inertia, coriolis accelerations and generated moments of the system derived from the model equations from the system. In this case, the only parameters of the connection that can be changed are the number of quadcopters and distances between them. All the quadcopters are aligned and their rotation around the $\mathrm{z}$-axis is always zero.

In the case of the architecture proposed in the current thesis, it is designed as PID controller in cascade that controls the position, linear velocities, attitudes and angular velocities. The gains of the proposed controller must be tuned for different distances, and then these values 
are interpolated to be used in every possible configuration of the system, or number of quadcopters. They developed not only the control strategy and architecture, but also an obstacle avoidance system and collaborative trajectory planning. Their strategy to control the system is based on a leader-follower method in which the leader sends the calculated set-points to the followers in real time. While in the proposed strategy in the current thesis, the set-points are sent simultaneously to all the quadcopters in the system. A VICON system was used as the only positioning system. VICON system is a camera-based motion capture system used, among other tasks, for object tracking [73].

In [41] the system is also composed of two rigidly attached quadcopters, that use individual cameras to estimate the position of each of the quadcopters, Fig. 1.5(b). Similarly to the previous example, the control architecture is based on a proportional controller in which the dynamical equations of the system are included so that the control architecture is adapted to every possible configuration, including the expression of the inertia and coriolis accelerations. The novelty in their approach is the optimization algorithm that they run in real time in both quadcopters to individually estimate the pose of the system of attached quadcopters while reducing the discrepancies between these estimations. In the current thesis, this problem is solved as only one of the quadcopters, the leader, will measure its position, and using the constraints derived from the physical connection between the quadcopters it will estimate the position of the system. Finally, this position is spread to the rest of quadcopters.

The control architecture proposed in [50], Fig. 1.5(c), is slightly more complex than in the previous examples. The position is controlled using a PID controller while for the attitude, a PD controller is used. Their architecture includes a transformation of the final control inputs in order to adapt it to the current positions and orientations of the quadcopters, derived from the pseudo inverse of the dynamics. This transformation takes into consideration the number of quadcopters, their positions and rotations around the z-axis. The attachment is rigid and the positioning is based on the VICON system, knowing each quadcopter their exact individual positions and orientations at every time-step. They even included a method to automatically attach or detach the load.

In [63], the most recent solution, a novel method to self-assemble the system of attached quadcopters in mid-air. Their controller struc- 


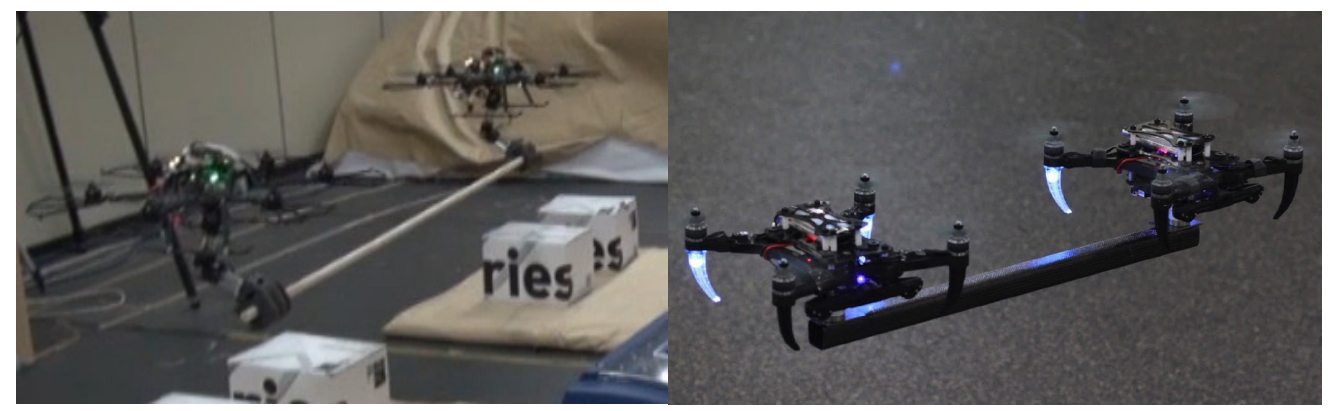

(a)

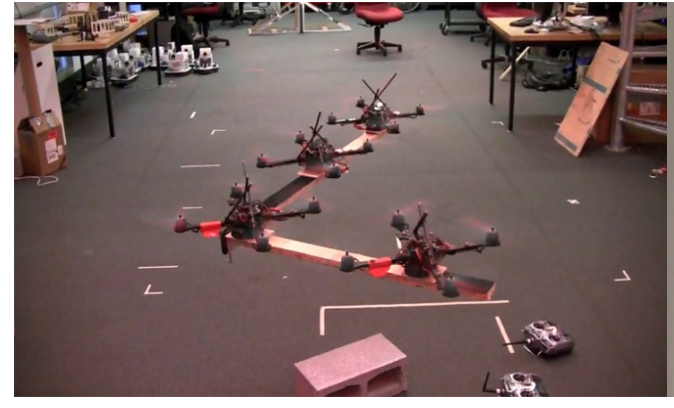

(c) (b)

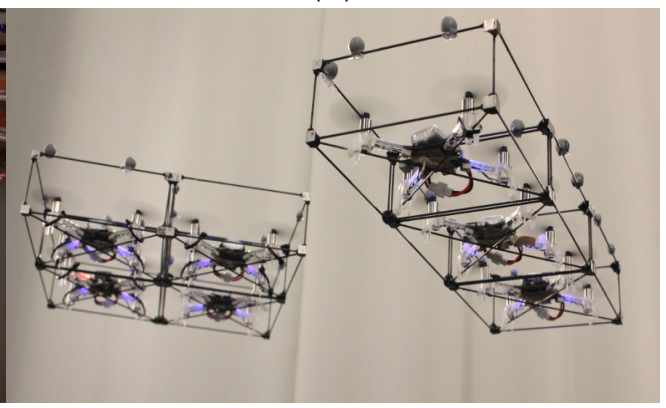

(d)

Figure 1.5: (a) Planning and Control for Collision-Free Cooperative Aerial Transportation [38]. (b) Cooperative Transportation Using Small Quadrotors Using Monocular Vision and Inertial Sensing [41]. (c) Cooperative Grasping and Transport Using Multiple Quadrotors [50]. (d) ModQuad: The Flying Modular Structure that Self-Assembles in Midair [63].

ture is based on the one proposed in [50], however, in this case they included an optimization method so that the thrust applied in each motor is the minimum possible, avoiding to saturate some motors depending on their position relative to the center of mass of the system. The control architecture includes a PD controller for the attitude and a proportional controller for the position and linear velocities control. As in the previous example, the individual positions and rotations of each quadcopters are measured in real time using the VICON tracking system.

The common factor of the proposed solutions is that the position and orientation of each quadcopter is known a priori ([41], [38]) or it is measured in real time ([50][63]). In the current thesis, a novel method is proposed to estimate the position and orientation of each 


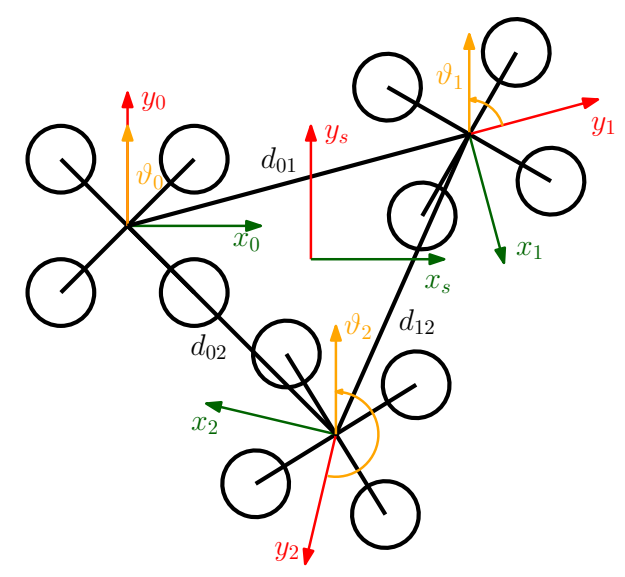

Figure 1.6: Simplified graphical representation of one possible configuration of the system of drones

quadcopter previous to any flight, with no a priori information and using only the on-board IMUs.

\subsection{Scope}

Current thesis' scope can be divided in different goals. First, the aim is to study the dynamics of a system of rigidly attached quadcopters including the physical parameters of the connection: number of quadcopters, their positions, and orientations. An example of a possible distribution, and the main variables that need to be studied, is depicted in Fig. 1.6 .

Once the dynamics of the system has been studied in terms of these main variables, the next step will be to design a method to estimate the distances between each pair of quadcopters $d_{i j}$ and their rotation around the z-axis $\vartheta_{i}$, using only the information from the individual on-board sensors.

Finally, a control strategy to ensure system stability and trajectory following capabilities for every possible configuration of the system of rigidly attached quadcopters. A global positioning system approach is also proposed. This approach allows to use both onboard sensors, as in [41], or other positioning methods such as Ultra Wide Band (UWB) systems or Motion Capture systems. The last goal is to design carefully the experimentation approach in order to validate the proposed methods. 


\subsection{Outline}

The ouline of the thesis will follow the same structure as the one proposed in the scope. First, the main theory related to the proposed methods is briefly introduced in Chapter 2 .

In Chapter 3, the model of the system of attached quadcopters is obtained. In Chapter 4 , the characteristics, concepts and mathematical development for the Physical Parameters Estimation step are explained. Right after, in Chapter 5, the proposed control architecture and strategy is developed.

The experimental setup is described in Chapter 6, where the hardware, implemented software and communication are explained. The summarized analysis of the obtained results is written in Chapter 7.

Finally, this analysis leads to the final conclusion and future work development, described in Chapter 8 . 


\section{Chapter 2}

\section{Preliminaries}

All the theory behind the methods that were used for the development of the current thesis is explained in detail.

\subsection{Notation}

- The operator $[\cdot]_{i}$ denotes the $i$-th element of a vector.

- The operator $[\cdot]_{i j}$ denotes the $i j$-th element of a matrix.

- The operator [.] denotes the first time derivative.

- The operator $[\ddot{\cdot}]$ denotes the second time derivative.

- The operator - denotes the dot product between two vectors or matrices.

- The operator $\times$ denotes the cross product between two vectors or matrices.

- The operator $\|\cdot\|$ denotes the Euclidean norm of a vector and the corresponding induced norm of a matrix.

\subsection{Quadcopter Modeling}

The model of a single quadcopter is well known, and the documentation related to this topic is wide [26][37][46]. The state of a quadcopter is represented by the position $\left[x^{t}, y^{t}, z^{t}\right] \mathrm{m}$, the linear velocities 


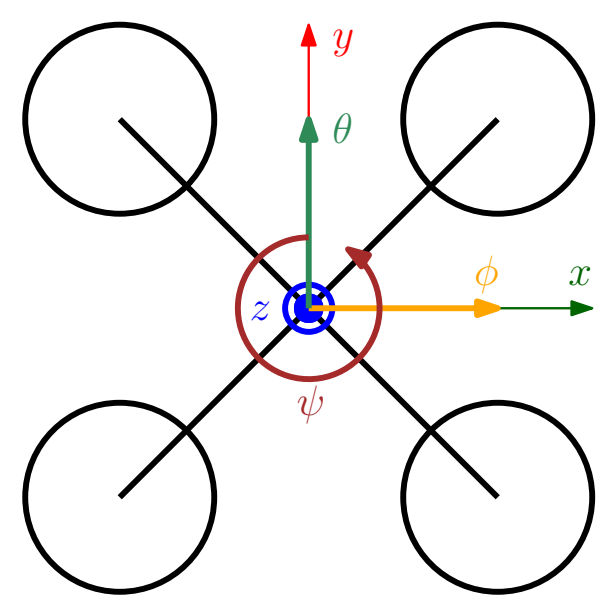

Figure 2.1: Simplified graphical representation of the ENU(East, North, Up) frame

$\left[\dot{x}^{t}, \dot{y}^{t}, \dot{z}^{t}\right] \frac{\mathrm{m}}{\mathrm{s}}$, the attitude or rotation around each axis $\left[\phi^{t}, \theta^{t}, \psi^{t}\right] \mathrm{rad}$, and the angular velocities $\left[w_{x}^{t}, w_{y}^{t}, w_{z}^{t}\right] \frac{\mathrm{rad}}{\mathrm{s}}$, see Fig. 2.1. The input to the system is the applied force by each of the propellers of the quadcopter. This force will be proportional to the voltage applied to each propeller which is controlled by Pulse-Width Modulation signals (PWM, [44]). Then, the single quadcopter system can be presented as:

$$
\left[x^{t}, y^{t}, z^{t}, \dot{x}^{t}, \dot{y}^{t}, \dot{z}^{t}, \phi^{t}, \theta^{t}, \psi^{t}, w_{x}^{t}, w_{y}^{t}, w_{z}^{t}\right]^{\top}=f\left(\mathrm{PWM}_{i}^{t}\right)
$$

where $\mathrm{PWM}_{i}^{t}$ is the PWM value of propeller $i$ in the current time step $t$. Then, the functions of the applied torque $Q_{i}\left(\mathrm{PWM}_{i}\right)$ and thrust $T_{i}\left(\mathrm{PWM}_{i}\right)$ can be defined in terms of the PWM value of the propeller $i$, see Fig. 2.2. These equations have to be determined for every quadcopter as it depends of the individual physical characteristics.

\subsubsection{Coordinate Frame and Rotation Matrix}

The coordinate frame orientation has to be defined previously. The ENU (East, North, Up) coordinate frame will be used (Fig. 2.1).

In the development of the equations the following symbols will be used: $p$ the position vector, $\mathrm{v}$ the velocity vector, $\Phi$ the attitude vector and $\boldsymbol{\omega}$ the angular velocities vector. The position, linear velocities and applied forces $F$ of the quadcopter can be expressed relative to the inertial, or world frame, or to the body frame. The superscript $B$ will refer 


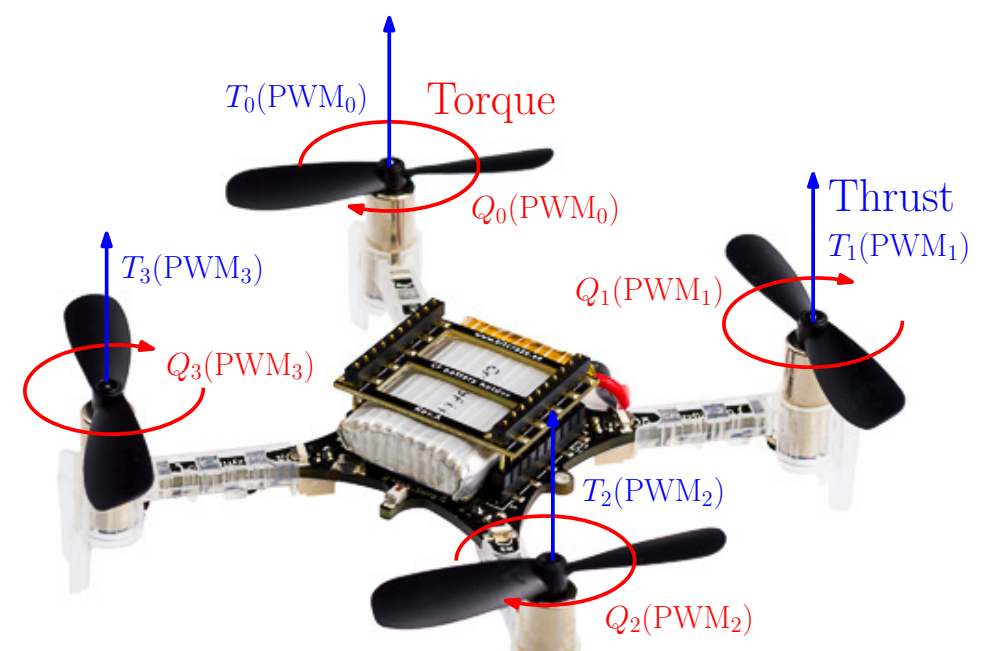

Figure 2.2: Graphical representation of the thrust and torque in a quadcopter

to the body frame and the superscript $I$ to the inertial frame. Then, the transformation matrices that map a vector expressed in the body frame $B$ to its expression in the inertial frame $I$ and vice versa are defined. The rotation matrix, from the inertial frame $I$ to the body frame $B$, can be obtained by performing three successive rotations:

$$
\begin{aligned}
\mathbf{R}_{I}^{B}=\mathbf{R}_{z}(\psi) \cdot \mathbf{R}_{y}(\theta) \cdot \mathbf{R}_{x}(\phi) \\
\mathbf{R}_{I}^{B}=\left[\begin{array}{ccc}
1 & 0 & 0 \\
0 & \cos (\phi) & \sin (\phi) \\
0 & -\sin (\phi) & \cos (\phi)
\end{array}\right] \cdot \\
{\left[\begin{array}{ccc}
\cos (\theta) & 0 & -\sin (\theta) \\
0 & 1 & 0 \\
\sin (\theta) & 0 & \cos (\theta)
\end{array}\right] \cdot\left[\begin{array}{ccc}
\cos (\psi) & \sin (\psi) & 0 \\
-\sin (\phi) & \cos (\psi) & 0 \\
0 & 0 & 1
\end{array}\right] }
\end{aligned}
$$


and the final expression is:

$$
\mathbf{R}_{I}^{B}=\left[\begin{array}{ccc}
\cos (\theta) \cos (\psi) & \cos (\theta) \sin (\psi) & -\sin (\theta) \\
\sin (\phi) \sin (\theta) \cos (\psi)-\cos (\phi) \sin (\psi) & \sin (\phi) \sin (\theta) \sin (\psi)+\cos (\phi) \cos (\psi) & \sin (\phi) \cos (\theta) \\
\cos (\phi) \sin (\theta) \cos (\psi)+\sin (\phi) \sin (\psi) & \cos (\phi) \sin (\theta) \sin (\psi)-\sin (\phi) \cos (\psi) & \cos (\phi) \cos (\theta)
\end{array}\right] .
$$

In order to transform a vector from the body frame $B$ to the inertial frame $I$ the inverse of $\mathbf{R}_{I}^{B}$ must be calculated. By definition, a rotation matrix has the following property [19]:

$$
\left(\mathbf{R}_{I}^{B}\right)^{-1}=\left(\mathbf{R}_{I}^{B}\right)^{T}=\mathbf{R}_{B}^{I}
$$

\subsubsection{Force Equations}

The expression of the force can be written, according to Newton's Second Law, as:

$$
\mathbf{F}_{\text {prop }}^{I}-\mathbf{F}_{g}^{I}-\mathbf{F}_{\text {cor }}^{I}-\mathbf{F}_{\text {fri }}^{I}=m a=m \ddot{\mathbf{x}}^{I} .
$$

$\mathbf{F}_{\text {prop }}^{I}$ is the force generated by the propellers, $\mathbf{F}_{g}^{I}$ the gravity force, $\mathbf{F}_{\text {cor }}^{I}$ the force due to the Coriolis acceleration and $\mathbf{F}_{\text {fri }}^{I}$ the air friction force component, all of them relative to the inertial $I$ relative frame. With $m$ the mass of the system and $a$ the acceleration, the individual expressions of the force can be developed:

$$
\left[\begin{array}{c}
F_{x}^{I} \\
F_{y}^{I} \\
F_{z}^{I}
\end{array}\right]-m\left[\begin{array}{c}
0 \\
0 \\
g_{z}
\end{array}\right]-m\left(\left[\begin{array}{c}
w_{x}^{I} \\
w_{y}^{I} \\
w_{z}^{I}
\end{array}\right] \times\left[\begin{array}{c}
v_{x}^{I} \\
v_{y}^{I} \\
v_{z}^{I}
\end{array}\right]\right)-\left[\begin{array}{ccc}
D_{1} & 0 & 0 \\
0 & D_{2} & 0 \\
0 & 0 & D_{3}
\end{array}\right]^{I} \cdot\left[\begin{array}{c}
v_{x}^{I} \\
v_{y}^{I} \\
v_{z}^{I}
\end{array}\right]=m \ddot{\mathbf{x}}^{I},
$$

where $F_{z}$ is the total force generated by the propellers and the matrix $D^{I}$ is the drag coefficients matrix both relative to the inertial frame. The force applied by the propellers is calculated in terms of the body frame $B$ but has to be expressed relative to the world frame $I$. This is achieved using the rotation matrices defined in Eq. 2.4 and Eq. 2.5

$$
\left[\begin{array}{c}
F_{x}^{I} \\
F_{y}^{I} \\
F_{z}^{I}
\end{array}\right]=\left(\mathbf{R}_{I}^{B}\right)^{-1} \cdot\left[\begin{array}{c}
F_{x}^{B} \\
F_{y}^{B} \\
F_{z}^{B}
\end{array}\right]=\left(\mathbf{R}_{I}^{B}\right)^{T} \cdot\left[\begin{array}{l}
F_{x}^{B} \\
F_{y}^{B} \\
F_{z}^{B}
\end{array}\right]
$$


Then Eq. 2.7 can be written as:

$$
\begin{aligned}
\dot{\mathbf{v}}^{I}= & \left(\mathbf{R}_{I}^{B}\right)^{T} \cdot \frac{1}{m}\left[\begin{array}{c}
F_{x}^{B} \\
F_{y}^{B} \\
F_{z}^{B}-g_{z}
\end{array}\right]-\left(\left[\begin{array}{c}
w_{x} \\
w_{y} \\
w_{z}
\end{array}\right] \times\left[\begin{array}{c}
v_{x}^{I} \\
v_{y}^{I} \\
v_{z}^{I}
\end{array}\right]\right) \\
& -\frac{1}{m}\left(\mathbf{R}_{I}^{B}\right)^{T} \cdot\left[\begin{array}{ccc}
D_{1} & 0 & 0 \\
0 & D_{2} & 0 \\
0 & 0 & D_{3}
\end{array}\right]^{B} \cdot\left[\begin{array}{c}
v_{x}^{I} \\
v_{y}^{I} \\
v_{z}^{I}
\end{array}\right]
\end{aligned}
$$

Vector $\mathbf{F}_{i}^{B}$ represents the thrust force produced by the motor $i$ of the quadcopter and can be considered null in the $\mathrm{x}$ and $\mathrm{y}$ dimensions as the forces generated in this axis due to the momentum in the $\mathrm{z}$-axis is negligible compared to the vertical thrust. Then, this vector has only a non-null component in the z-component, $F_{z}^{B}$, being:

$$
\mathbf{F}_{z}^{B}=\sum_{i=0}^{4} \mathbf{F}_{i}^{B}=\sum_{i=0}^{4}\left[\begin{array}{c}
0 \\
0 \\
T_{i}\left(\mathrm{PWM}_{i}\right)
\end{array}\right]
$$

\subsubsection{Momentum Equations}

Using the Coriolis equation, the total angular momentum is expressed:

$$
\sum_{i=1}^{K} \mathbf{M}_{i}=\dot{\mathbf{h}}+\mathbf{w} \times \mathbf{h},
$$

where $\mathbf{M}_{i}$ represents each of the $\mathrm{K}$ momentums applied to the system, $\mathbf{h}$ is the angular momentum around the quadcopter's center of gravity. According to the Angular Momentum theory, this momentum is proportional to the Inertia $\mathbf{J}$ and angular velocities $\mathbf{w}$ of a system:

$$
\mathbf{h}=\mathbf{J} \mathbf{w}
$$

In this case, the goal is to calculate the angular velocities and attitude Euler angles relative to the body frame. Then the expression 2.11 is developed as: 


$$
\sum_{i=1}^{K} \mathbf{M}_{i}=\mathbf{J}^{B} \dot{\mathbf{w}}^{B}+\mathbf{w}^{B} \times \mathbf{J}^{B} \mathbf{w}^{B},
$$

where $\mathbf{J}^{B}$ is the inertia matrix of the quadcopter expressed in the body frame. This matrix has to be calculated for each different quadcopter. Using this equation, the vector $\dot{\mathbf{w}}^{B}$ can be obtained:

$$
\dot{\mathbf{w}}^{B}=\left(\mathbf{J}^{B}\right)^{-1}\left(\sum_{i=1}^{K} \mathbf{M}_{i}-\mathbf{w}^{B} \times \mathbf{J}^{B} \mathbf{w}^{B}\right),
$$

also expressed as:

$$
\left[\begin{array}{c}
\dot{w}_{x}^{B} \\
\dot{w}_{y}^{B} \\
\dot{w}_{z}^{B}
\end{array}\right]=\left(\mathbf{J}^{B}\right)^{-1}\left(\left[\begin{array}{c}
M_{x}^{B} \\
M_{y}^{B} \\
M_{z}^{B}
\end{array}\right]-\left[\begin{array}{c}
w_{x}^{B} \\
w_{y}^{B} \\
w_{z}^{B}
\end{array}\right] \times \mathbf{J}^{B}\left[\begin{array}{l}
w_{x}^{B} \\
w_{y}^{B} \\
w_{z}^{B}
\end{array}\right]\right) .
$$

Using $\mathbf{p}_{i}^{B}$ for the position of the motor $i$ in the body frame, and $\mathbf{F}_{i}^{B}$ for the aerodynamical force generated by the propeller $i$ in the body frame and $\mathbf{Q}_{i}^{B}$ for the torque, then:

$$
\mathbf{M}=\sum_{i=0}^{4} \mathbf{p}_{i}^{B} \times \mathbf{F}_{i}^{B}+\sum_{i=0}^{4} \mathbf{Q}_{i}^{B} .
$$

The cross product between $\mathbf{p}_{i}^{B}$ and the force $\mathbf{F}_{i}^{B}$ can be written as:

$$
\sum_{i=0}^{4} \mathbf{p}_{i}^{B} \times \mathbf{F}_{i}^{B}=\sum_{i=0}^{4}\left[\begin{array}{c}
-F_{z_{i}}^{B}\left|p_{y_{i}}^{B}\right| \\
F_{z_{i}}^{B}\left|p_{x_{i}}^{B}\right| \\
0
\end{array}\right]=\sum_{i=0}^{4}\left[\begin{array}{c}
-T_{i}\left(\mathrm{PWM}_{i}\right)\left|p_{y_{i}}^{B}\right| \\
T_{i}\left(\mathrm{PWM}_{i}\right)\left|p_{x_{i}}^{B}\right| \\
0
\end{array}\right]
$$

The generated torque around the z-axis, depends on the rotational direction of the propellers and the expression of the torque, $Q_{i}\left(\mathrm{PWM}_{i}\right)$ :

$$
\sum_{i=0}^{4} \mathbf{Q}_{i j}^{B}=\sum_{i=0}^{4}\left[\begin{array}{c}
0 \\
0 \\
s_{i j} Q_{i}\left(\mathrm{PWM}_{i}\right)
\end{array}\right]
$$


where $s_{i j}$ is the rotational direction's sign of the propeller $i$ based on anti-clockwise convection or rule of the thumb. Then, a clock-wise rotation will correspond to a negative sign $s_{i j}$ and vice versa.

Finally, Eq. 2.16 is re-written:

$$
\mathbf{M}=\left[\begin{array}{c}
M_{x}^{B} \\
M_{y}^{B} \\
M_{z}^{B}
\end{array}\right]=\sum_{i=0}^{4}\left[\begin{array}{c}
-T_{i}\left(\mathrm{PWM}_{i}\right)\left|p_{y_{i}}^{B}\right| \\
T_{i}\left(\mathrm{PWM}_{i}\right)\left|p_{x_{i}}^{B}\right| \\
s_{i} Q_{i}\left(\mathrm{PWM}_{i}\right)
\end{array}\right]
$$

where the thrust $T_{i}\left(\mathrm{PWM}_{i}\right)$ and torque $Q_{i}\left(\mathrm{PWM}_{i}\right)$ of each propeller $i$ are expressed in terms of the PWM input to that propeller. To obtain this relation between the torque and thrust with the PWM values of each propeller it is necessary to map first the angular speed of the propellers to the correspondent thrust and momentum generated. To do so, and following the fluid mechanics formula developed in [16], the expressions of the torque $Q$ and the thrust $T$ are written:

$$
\begin{gathered}
T_{i}\left(n_{i}\right)=C_{T}\left(n_{i}\right) \rho n_{i}^{2} D^{4} \\
P_{i}\left(n_{i}\right)=C_{P}\left(n_{i}\right) \rho n_{i}^{3} D^{5} \\
Q_{i}\left(n_{i}\right)=\frac{P_{i}\left(n_{i}\right)}{2 \pi n_{i}},
\end{gathered}
$$

where $n_{i}$ is the rotations per second, $D$ the diameter of the propellers, $\rho$ the density of the air, P the generated energy by the propellers. The parameters $C_{T}(n)$ and $C_{P}(n)$ are the so called thrust and power coefficients.

The positions of each motor in a single quadcopter can be expressed as:

$$
\left[\begin{array}{llll}
\mathbf{p}_{0} & \mathbf{p}_{1} & \mathbf{p}_{2} & \mathbf{p}_{3}
\end{array}\right]=\left[\left[\begin{array}{c}
L / \sqrt{2} \\
-L / \sqrt{2} \\
0
\end{array}\right]\left[\begin{array}{c}
-L / \sqrt{2} \\
-L / \sqrt{2} \\
0
\end{array}\right]\left[\begin{array}{c}
-L / \sqrt{2} \\
L / \sqrt{2} \\
0
\end{array}\right]\left[\begin{array}{c}
L / \sqrt{2} \\
L / \sqrt{2} \\
0
\end{array}\right]\right]
$$

with $L$ being the distance between a motor and the quadcopter's center of gravity. 
Finally, the vector $\dot{\mathbf{w}}^{B}$ can be calculated using Eq. 2.15. Using this vector, the attitude Euler angles derivative $\dot{\phi}$ is estimated:

$$
\left[\begin{array}{c}
\dot{\phi} \\
\dot{\theta} \\
\dot{\psi}
\end{array}\right]=\left[\begin{array}{ccc}
1 & \sin (\phi) \tan (\theta) & \cos (\phi) \tan (\theta) \\
0 & \cos (\phi) & -\sin (\phi) \\
0 & \sin (\phi) / \cos (\theta) & \cos (\phi) / \cos (\theta)
\end{array}\right]\left[\begin{array}{c}
w_{x}^{B} \\
w_{y}^{B} \\
w_{z}^{B}
\end{array}\right] \text { for } \theta \neq \frac{\pi}{2}
$$

\subsubsection{Integration of the Dynamical Model Equations}

Once all the equations are derived, the calculated parameters are integrated to obtain the current full-state of the system $[\mathbf{p}, \mathbf{v}, \boldsymbol{\Phi}, \boldsymbol{\omega}]$. Using all the equations expressed in the previous pages, is obtained:

$$
\dot{\mathbf{s}}(k-1)=\left[\begin{array}{c}
\dot{x}(k-1) \\
\dot{y}(k-1) \\
\dot{z}(k-1) \\
\ddot{x}(k-1) \\
\ddot{y}(k-1) \\
\ddot{z}(k-1) \\
\dot{\phi}(k-1) \\
\dot{\theta}(k-1) \\
\dot{\psi}(k-1) \\
\dot{w}_{x}(k-1) \\
\dot{w}_{y}(k-1) \\
\dot{w}_{y}(k-1)
\end{array}\right],
$$

where $k-1$ denotes the previous time step. Now, the expression of a derivative approximated using Newton's difference quotient [11]:

$$
\dot{x}=\frac{x(h k+h)-x(k h)}{h} \Rightarrow x(h k+h)=\dot{x} h+x(h k) .
$$

Then, using a time step of $h$ the value of $s(k)$ can be obtained:

$$
s_{i}(k)=\dot{s}_{i}(k-1) h+s_{i}(k-1),
$$


obtaining:

$$
\mathbf{s}(k)=\left[\begin{array}{c}
x(k) \\
y(k) \\
z(k) \\
\dot{x}(k) \\
\dot{y}(k) \\
\dot{z}(k) \\
\phi(k) \\
\theta(k) \\
\psi(k) \\
w_{x}(k) \\
w_{y}(k) \\
w_{y}(k)
\end{array}\right] .
$$

\subsection{PID Controller}

A Proportional-Integral-Derivative controller is a control loop feedback mechanism used in the field of robotics. The main idea is that, given the continuous error signal $e(t)$ obtained as the difference between the desired set-point and the current state, the controller applies a correction based on the proportional, derivative and integral values of this error [67]. The error signal can be expressed as:

$$
e(t)=x(t)-y(t)
$$

with $x(t)$ the desired output and $y(t)$ the measured output. The general expression of a PID controller is given by:

$$
u(t)=K_{p} e(t)+K_{i} \int_{0}^{t} e\left(t^{\prime}\right) d t^{\prime}+K_{d} \frac{d e(t)}{d t},
$$

where $K_{p}, K_{i}$ and $K_{d}$ are all non-negative, and denote the proportional, integral and derivative gains. $u(t)$ is the control action calculated by the controller. In discrete time, this PID controller can be expressed as:

$$
u(k)=K_{p} e(k)+K_{i}\left(\sum_{i=0}^{k} e(i)\right) \Delta k+K_{d} \frac{e(k)-e(k-1)}{\Delta k} .
$$


Each gain has the following characteristics:

- $K_{p}$ : Weights the value of the proportional term of the error. The bigger the error is, the bigger the control action will be. A very high proportional gain will lead to unstable responses while a very low one will lead to a small output response and a big error.

- $K_{i}$ : Weights the value of the integral term of the error. The contribution from the integral term is both the magnitude of the error and the duration of the error. Therefore, the integral term can be considered as the accumulative value of the error. The objective of this term is to eliminate this residual error.

- $K_{d}$ : Weights the value of the derivative term of the error. This term, can be understood as an estimate of the future errors, based on the current slope, or change rate, of the error. It reduces the overshooting in the system.

\subsection{Neural Networks}

Neural networks can be understood as a set of mathematical algorithms, based on the human brain neural processes, that were mainly designed for pattern recognition and classification [78][28]. They are commonly used in classification tasks (face or speech recognition), clustering, or predictive analysis, like hardware failure prediction or the likelihood of a customer to leave.

Neurons in the human brain, propagate a signal only when they get excited by a received signal stronger than a threshold (action potential threshold [42]). The strength of the propagated signal between two neurons is proportional to the strength of the connection between them. Similarly, Neural Networks are composed of units that will propagate a signal depending on the value of the input signal. The strength of the communication between two units is defined by a weight value assigned to each connection. To emulate the behavior of the biological neurons, these units have a function that transforms the input signal into the output signal. While these functions remain unchanged, the weights between the units are variable. The training of a Neural Network is the process of tuning these weights to obtained the desired behavior from the Neural Network [13]. 


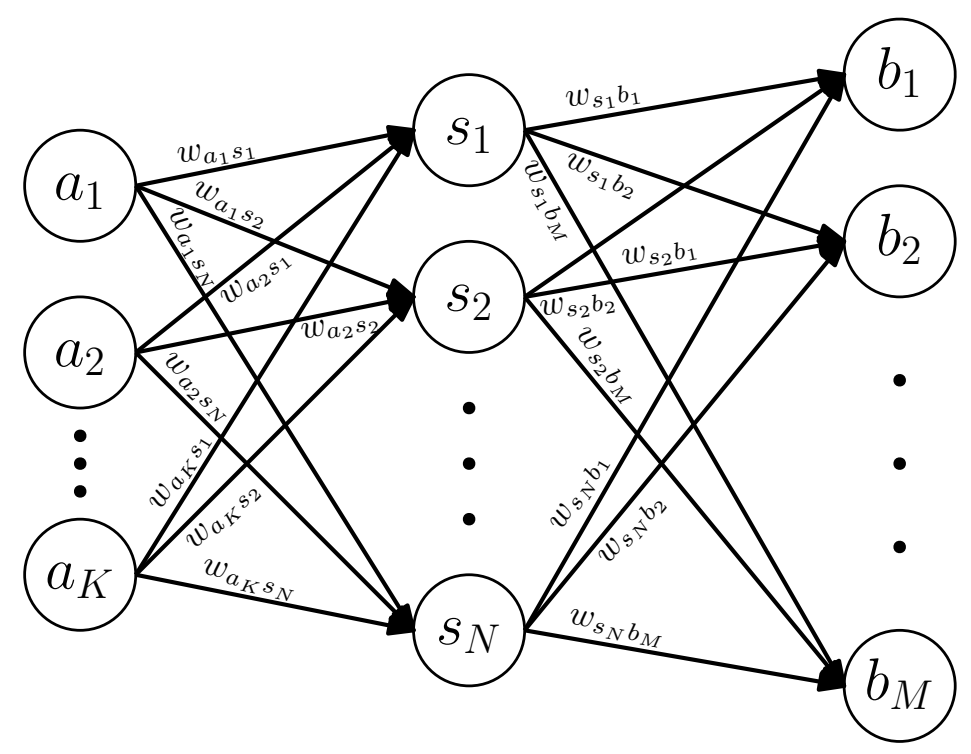

Figure 2.3: Simple example of a Neural Network.

A simple example of the structure of a Neural Network is shown in Fig. 2.3. In this figure, the inputs are represented by $a_{i}$ and the outputs as $b_{i}$, while the hidden layers, form by this "neural" units are denoted by $s_{i}$. The weight between units is expressed as $w_{i j}$.

To train the Network, an error function $E$ must be defined over the set of pairs $\left(a_{1}, b_{1}\right), \ldots,\left(a_{N}, b_{N}\right)$. The weights are represented by $\Theta$, and the Neural Network by $g_{\Theta}(\mathbf{a})$. The error function can be defined by $E(A, \Theta)$, with the lowest value obtained when $g_{\Theta}\left(a_{i}\right)=b_{i}$. An example of this error function can be the Mean Squared Error:

$$
E(A, \Theta)=\sum_{i=1}^{N} \frac{\left(b_{i}-g_{\Theta}\left(a_{i}\right)\right)^{2}}{N}
$$

The parameters included in $\Theta$ are optimized using Gradient Descent with Backpropagation [30]. The objective of the gradient descent process is to obtain the gradient of $g_{\Theta}$ for a certain value of $\Theta$ and then update this value by stepping in the direction of the negative gradient. This process is done until a local minimum is reached. The term Backpropagation refers to the way this gradient is computed: to ensure convergence to a local minima for big networks, this gradient has to be calculated from the end of the network to beginning. The gradient of the final layer's weights is calculated first and the gradient of 
the first layer's weights calculated last.

\section{Activation Functions}

Each unit in a Neural Network has an activation function that transforms the input into the output, emulating the actual biological neurons behavior. There is a wide range of activation functions, however, this thesis will focus the Rectified Linear Units (ReLU) activation function, defined by:

$$
g_{\Theta}(x)=\max (0, x)
$$

\subsection{Reinforcement Learning}

Reinforcement Learning is a type of Machine Learning that allows machines and software agents to determine the optimal behavior within a specific environment in order to maximize their performance. It is a type of dynamic programming that trains algorithms using a system of reward and punishment. The main tool to achieve this maximized performance is the use of a reward feedback which aim is to allow the agent to learn its optimal behavior for a specific task under the arbitrary reward function that is defined. The environment, and therefore, the available actions and possible states of the agent are formulated as a Markov Decision Process (MDP), this means that the current state of the system depends uniquely on the previous state.

In general, Reinforcement Learning algorithms [32] define an Agent that can perform an action $a \in A$ that triggers the transition of the Agent from the state $s_{t}$ to the state $s_{t+1}, A$ being all the possible actions that can be taken by the Agent. Each state has associated a certain reward $s_{t} \Rightarrow r_{t}$, being the objective of every Reinforcement Learning algorithm to maximize this reward [33]. The optimal action $a_{t}$ for each state is the one that maximizes the long-term reward, Eq. 2.34.

There is a wide variety of existing Reinforcement learning methods. This thesis will focus on Q-learning, characterized by the implementation of $\mathrm{Q}$ function $Q(s, a)$ that maps the pairs of states $s$ and actions $a$ with their expected reward $r$. In the case of Q-learning, this reward is a weighted sum of the expected values of the rewards [74]:

$$
R_{t}=\gamma^{0} r_{t}+\gamma^{1} r_{t+1}+\gamma^{2} r_{t+2}+\ldots+\gamma^{n-t} r_{t+n}
$$


that can be also expressed as:

$$
R_{t}=r_{t}+\gamma R_{t+1}
$$

The policy $\pi(s)$ will decide what action to take next, and is expressed as:

$$
\pi(s)=\underset{a}{\operatorname{argmax}} Q(s, a) .
$$

As the objective is to maximize the reward, for a certain time step:

$$
Q\left(s_{t}, a_{t}\right)=\max R_{t+1}
$$

The Q-function can be rewritten as:

$$
Q\left(s_{t}, a_{t}\right)=r_{t}+\gamma \max _{a} Q\left(s_{t+1}, a_{t+1}\right) .
$$

Then for each pair of $a_{t}$ and $s_{t}$, there is an associated value of the expected reward $r_{t}$. To reach convergence, this $\mathrm{Q}$-function has to store all the possible states and actions for each state. Then, the main problem with this algorithm is that it is computational expensive as it needs to compute and store all this possible actions and states in a table. This algorithm needs a lot of time to reach convergence [74].

\subsubsection{Deep Q-Learning}

Q-learning can be understood as a function approximation problem in which the objective is to approximate the Q-function [3]. Then, this Qfunction can be used by the agent to choose the most optimal action for each state. Neural Networks have proved to perform very efficiently in the task of non-linear functions approximation [77][75]. Therefore, instead of building a table with all the possible combinations of states, actions and rewards, a Neural Network will be used with the objective of approximating the Q-function and simplify the problem. The basic characteristics of Neural Networks are introduced in Section 2.4. This approach is commonly called Deep Q-learning in which, instead of saving this possible values for the Q-function in a table, a Neural Network is used, and only the weights of this Neural Network are trained [53]. This Neural Network is called the Q-Network. If we defined weights of Q-Network as $\Theta$ then the Q-Network is expressed as:

$$
Q(s, a ; \Theta) \text {. }
$$


The inner structure of this network will depend on the characteristics of the environment that is wanted to be learned. However, the input and output number of units is determined by the the number of actions and characteristics of the state. The input length is equal to the state length, and the output equal to the number of possible actions. The main steps of the learning algorithm are [53]:

1. Given the current state $s$, perform a forward pass to the neural network $Q(s, a ; \theta)$ to get the predicted reward values for every action.

2. Trigger the transition to the next state $s^{\prime}$ by choosing one of the possible actions. This action can be chosen on-policy, as in Eq. 2.36 or randomly (off-policy).

3. Do a feedforward pass with the next state $s^{\prime}$ and to choose the on-policy action $a^{\prime}$ by doing $\max _{a^{\prime}} Q\left(s^{\prime}, a^{\prime}, \Theta\right)$.

4. Update the weights $\theta$ by performing Backpropagation, as in Section 2.4, with the following error function $E$ :

$$
E=\frac{1}{2}\left[r+\max _{a^{\prime}} Q\left(s, a^{\prime} ; \Theta\right)-Q(s, a ; \Theta)\right]
$$

\subsection{Kalman Filter}

The automatic control of a process requires the estimation of the state of this process. This estimation is complex, and not ideal: it is affected by noise and bias in the measurements. Besides, the measurements can not be obtained continuously from the digital sensors, or if they do, they are usually not processed continuously in the processing units. This lead to time steps in which the state of the system is completely unknown, which can drive the system to an unstable state.

To solve both issues, estimators are used to estimate states which cannot be measured or are not measured at all times and to filter noise in the measurements. For development of the methods proposed in the thesis, the implementation of a Kalman filter is required.

Kalman filter's algorithm [34] uses a series of measurements observed over time, containing white noise and other imperfections, such as bias, and produces the estimation of unknown variables. The main 
characteristic of the Kalman filter is the use of a joint probability distribution over the variables for each time-step.

With $\mathbf{x}_{k}$ the current state and $\mathbf{z}_{k}$ the current observation and $\mathbf{u}_{k}$ the current applied action, $\mathbf{F}_{k}$ the state-transition model, $\mathbf{H}_{k}$ the observation model, $\mathbf{Q}_{k}$ the covariance of the process noise, $\mathbf{R}_{k}$ the covariance of the observation noise, $\mathbf{B}_{k}$ the control input model, $\mathbf{w}_{k}$ the process noise, $\mathbf{v}_{k}$ the observation noise:

$$
\begin{aligned}
\mathbf{x}_{k} & =\mathbf{F}_{k} \mathbf{x}_{k-1}+\mathbf{B}_{k} \mathbf{u}_{k}+\mathbf{w}_{k} \\
\mathbf{z}_{k} & =\mathbf{H}_{k} \mathbf{x}_{k}+\mathbf{v}_{k}
\end{aligned}
$$

The expression of the a posteriori state estimate is introduced: $\hat{\mathbf{x}}_{m \mid n}$ represents the estimation of the state at time step $m$ using the $n$ first observations. In the same line, the a posteriori error covariance matrix is defined as $\mathbf{P}_{m \mid n}$. Then, the equations of the Kalman filter algorithm separated in Prediction equations and Update equations are expressed, in order:

- Predict Step:

$$
\begin{aligned}
\hat{\mathbf{x}}_{k \mid k-1} & =\mathbf{F}_{k} \hat{x}_{k-1 \mid k-1}+\mathbf{B}_{k} \mathbf{u}_{k}+\mathbf{w}_{k} \\
\mathbf{P}_{k \mid k-1} & =\mathbf{F}_{k} \mathbf{P}_{k-1 \mid k-1} \mathbf{F}_{k}^{\top}+\mathbf{Q}_{k}
\end{aligned}
$$

- Update Step:

$$
\begin{aligned}
\tilde{\mathbf{y}}_{k} & =\mathbf{z}_{k}-\mathbf{H}_{k} \hat{\mathbf{x}}_{k \mid k-1} \\
\mathbf{S}_{k} & =\mathbf{R}_{k}+\mathbf{H}_{k} \mathbf{P}_{k \mid k-1} \mathbf{H}_{k}^{\top} \\
\mathbf{K}_{k} & =\mathbf{P}_{k \mid k-1} \mathbf{H}_{k}^{\top} \mathbf{S}_{k}^{1} \\
\hat{\mathbf{x}}_{k \mid k} & =\hat{\mathbf{x}}_{k \mid k-1}+\mathbf{K}_{k} \tilde{\mathbf{y}}_{k} \\
\mathbf{P}_{k \mid k} & =\left(\mathbf{I}-\mathbf{K}_{k} \mathbf{H}_{k}\right) \mathbf{P}_{k \mid k-1}\left(\mathbf{I}-\mathbf{K}_{k} \mathbf{H}_{k}\right)^{\top}+\mathbf{K}_{k} \mathbf{R}_{k} \mathbf{K}_{k}^{\top} \\
\tilde{\mathbf{y}}_{k \mid k} & =\mathbf{z}_{k}-\mathbf{H}_{k} \hat{\mathbf{x}}_{k \mid k}
\end{aligned}
$$




\section{Chapter 3}

\section{Dynamical Model of Rigidly At- tached Quadcopters}

The dynamical model of the system of $N$ rigidly attached quadcopters is developed first. The main constraint for the system of rigidly attached quadcopters is, as stated in Section 1.4. that all of these quadcopters must be situated in the same plane and that the attachment is assumed to be ideally rigid. However, the values of the distances, relative rotations around the z-axis or number of quadcopters in the system are not defined $a$ priori. Therefore, the combination of number of quadcopters, relative distances, and relative rotations is infinite, and the model has to be written in a way that all the possible combinations are included. See in Fig. 3.1 a schematic representation of the system model for an arbitrary distribution of 3 quadcopters.

The model of the system now will depend on the three variables that has been recently mentioned: the individual position $\mathbf{P}_{i}^{o}$ and rotation around the z-axis $\vartheta_{i}^{o}$ of each quadcopter $i$ in the system $S$ and the number of quadcopters $N$. Now the full-state of the system of attached quadcopters can be expressed as:

$$
\left[x_{s}^{t}, y_{s}^{t}, z_{s}^{t}, \dot{x}_{s}{ }^{t}, \dot{y}_{s}^{t}, \dot{z}_{s}^{t}, \phi_{s}^{t}, \theta_{s}^{t}, \psi_{s}^{t}, w_{x_{s}}^{t} w_{y_{s}}^{t}, w_{z_{s}}^{t}\right]^{\top}=f_{s}\left(\mathbf{P}_{i}^{o} \ldots, \vartheta_{i}^{o} \ldots, \mathrm{PWM}_{i, j}^{t} \ldots\right),
$$

where $s$ denotes the system's coordinate frame, $\mathbf{P}_{i}^{o}$ refers to the position $\left[x_{o i}^{t}, y_{o i}^{t}, z_{o i}^{t}\right]$ of the quadcopter $i, \vartheta_{i}^{o}$ denotes the rotation around the $\mathrm{z}$ axis for the quadcopter $i$, and $\mathrm{PWM}_{i, j}^{t}$ the PWM (voltage) applied to the motor $j$ of the quadcopter $i$, relative to the current time step $t$.

The coordinate frames' orientation will be identical as in the single quadcopter case, the ENU (East, North, Up) frame, see Fig. 2.1. The 


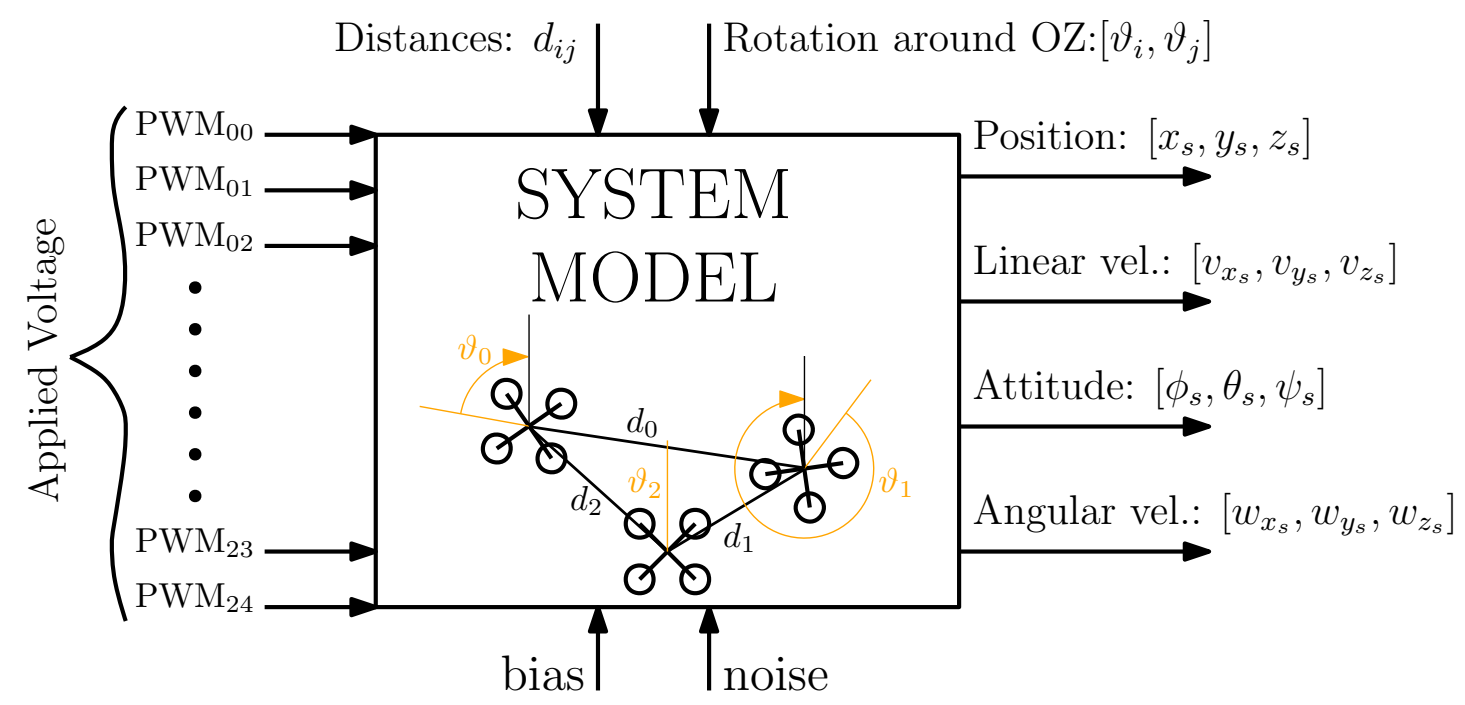

Figure 3.1: Schematic representation of the system model for an arbitrary distribution of 3 quadcopters.

development of the equations will follow the same nomenclature as in Section 2.2

\subsection{Force Equations}

The force equations are based on Eq. 2.9.

$$
\begin{aligned}
\dot{\mathbf{v}}_{s}^{B}= & \frac{1}{m_{s}}\left(\mathbf{R}_{I}^{B}\right)^{\top}\left[\begin{array}{c}
F_{x_{s}}^{B} \\
F_{y_{s}}^{B} \\
F_{z_{s}}^{B}
\end{array}\right]-\left[\begin{array}{l}
0 \\
0 \\
g_{z}
\end{array}\right] \\
& -\left(\left[\begin{array}{l}
w_{x_{s}} \\
w_{y_{s}} \\
w_{z_{s}}
\end{array}\right] \times\left[\begin{array}{c}
v_{x_{s}}^{I} \\
v_{y_{s}}^{I} \\
v_{z_{s}}^{I}
\end{array}\right]\right)-\frac{1}{m_{s}}\left[\begin{array}{ccc}
D_{1}^{s} & 0 & 0 \\
0 & D_{2}^{s} & 0 \\
0 & 0 & D_{3}^{s}
\end{array}\right]^{I}\left[\begin{array}{c}
v_{x_{s}}^{I} \\
v_{y_{s}}^{I} \\
v_{z_{s}}^{I}
\end{array}\right]
\end{aligned}
$$

where $F_{z_{s}}$ is the total force generated by the $4 N$ propellers and the matrix $D_{I}^{s}$ is the drag coefficients matrix of $S$ relative to the inertial frame, obtained by:

$$
\left[\begin{array}{ccc}
D_{1}^{s} & 0 & 0 \\
0 & D_{2}^{s} & 0 \\
0 & 0 & D_{3}^{s}
\end{array}\right]^{I}=\left(\mathbf{R}_{I}^{B}\right)^{\top}\left[\begin{array}{ccc}
D_{1}^{s} & 0 & 0 \\
0 & D_{2}^{s} & 0 \\
0 & 0 & D_{3}^{s}
\end{array}\right]^{B}=\left(\mathbf{R}_{I}^{B}\right)^{\top} \sum_{i=1}^{N}\left[\begin{array}{ccc}
D_{1}^{i} & 0 & 0 \\
0 & D_{2}^{i} & 0 \\
0 & 0 & D_{3}^{i}
\end{array}\right]^{I}
$$


The vector $F_{i j}^{B}$ represents the thrust force produced by the motor $j$ of the $i$-th quadcopter of $S$. Then $F_{z}^{B}$ is dependent on the thrust $T_{i j}\left(\mathrm{PWM}_{i j}\right)$ generated by each propeller $i j$ :

$$
\mathbf{F}_{i j}^{B}=\left[\begin{array}{c}
0 \\
0 \\
T_{i j}\left(\mathrm{PWM}_{i j}\right)
\end{array}\right] \rightarrow \mathbf{F}^{B}=\sum_{i=0}^{N} \sum_{j=0}^{4}\left[\begin{array}{c}
0 \\
0 \\
T_{i j}\left(\mathrm{PWM}_{i j}\right)
\end{array}\right]
$$

\subsection{Momentum Equations}

As in Section 2.2, the angular acceleration of the system $S$ can be obtained by the following equation:

$$
\left[\begin{array}{c}
\dot{w}_{x_{s}}^{B} \\
\dot{w}_{y_{s}}^{B} \\
\dot{w}_{z_{s}}^{B}
\end{array}\right]=\left(\mathbf{J}_{S}^{B}\right)^{-1}\left(\left[\begin{array}{c}
M_{x_{s}}^{B} \\
M_{y_{s}}^{B} \\
M_{z_{s}}^{B}
\end{array}\right]-\left[\begin{array}{c}
w_{x_{s}}^{B} \\
w_{y_{s}}^{B} \\
w_{z_{s}}^{B}
\end{array}\right] \times \mathbf{J}_{S}^{B}\left[\begin{array}{l}
w_{x_{s}}^{B} \\
w_{y_{s}}^{B} \\
w_{z_{s}}^{B}
\end{array}\right]\right)
$$

With $\mathbf{p}_{i j}^{B}=\left[p_{x_{i j}}^{B}, p_{y_{i j}}^{B}\right]$ expressing the position of the motor $i j$ in the body frame, $\mathbf{F}_{i j}^{B}$ the aerodynamical force generated by the propeller $i j$ in the body frame and $\mathbf{Q}_{i j}^{B}$ the torque, then:

$$
\mathbf{M}_{S}=\sum_{i=0}^{N} \sum_{j=0}^{4} \mathbf{p}_{i j}^{B} \times \mathbf{F}_{i j}^{B}+\sum_{i=0}^{N} \sum_{j=0}^{4} \mathbf{Q}_{i j}^{B}
$$

where $N$ is the total number of quadcopters in the system. This expression was already obtained in Eq. 2.19, and for the system $S$ of $\mathrm{N}$ quadcopters, it can be written as:

$$
\mathbf{M}_{S}=\left[\begin{array}{c}
M_{x}^{B} \\
M_{y}^{B} \\
M_{z}^{B}
\end{array}\right]=\sum_{i=0}^{N} \sum_{j=0}^{4}\left[\left[\begin{array}{c}
-T_{i j}\left(\mathrm{PWM}_{i j}\right)\left|p_{y_{i j}}^{B}\right| \\
T_{i j}\left(\mathrm{PWM}_{i j}\right)\left|p_{x_{i j}}^{B}\right| \\
s_{i j} Q_{i j}\left(\mathrm{PWM}_{i j}\right)
\end{array}\right]\right] .
$$

Each motor's position $\mathbf{p}_{i}^{B}$ can be calculated using the position of the motors $j$ within the body frame of a single quadcopter $\mathbf{p}_{j}=\left[p_{x_{j}}, p_{y_{j}}\right]$ and the position $\mathbf{P}_{i}^{o}$ and rotation $\vartheta_{i}^{o}$ of each quadcopter in the system $S$ relative to the body frame $B$. With the positions of each motor in 


\section{QUADCOPTERS}

a single quadcopter expressed in Eq. 2.23, the position of each motor expressed in the system's $S$ body frame is:

$$
\mathbf{p}_{i j}^{B}=\left[\begin{array}{ccc}
\cos \left(\vartheta_{i}^{o}\right) & \sin \left(\vartheta_{i}^{o}\right) & 0 \\
-\sin \left(\vartheta_{i}^{o}\right) & \cos \left(\vartheta_{i}^{o}\right) & 0 \\
0 & 0 & 1
\end{array}\right] \cdot \mathbf{p}_{j}+\mathbf{P}_{i}^{o} .
$$

The vector $\dot{\mathbf{w}}_{s}^{B}$ can be calculated using Equation 3.5 . Using this vector, the attitude Euler angles derivative $\dot{\phi}$ is estimated:

$$
\left[\begin{array}{c}
\dot{\phi} \\
\dot{\theta} \\
\dot{\psi}
\end{array}\right]=\left[\begin{array}{ccc}
1 & \sin (\phi) \tan (\theta) & \cos (\phi) \tan (\theta) \\
0 & \cos (\phi) & -\sin (\phi) \\
0 & \frac{\sin (\phi)}{\cos (\theta)} & \frac{\cos (\phi)}{\cos (\theta)}
\end{array}\right]\left[\begin{array}{c}
w_{x_{s}}^{B} \\
w_{y_{s}}^{B} \\
w_{z_{s}}^{B}
\end{array}\right] \text { for } \theta \neq \frac{\pi}{2}
$$

\subsection{Inertia Matrix}

The last unknown that needs to be obtained is the value of the inertia matrix depending of the distribution of the $N$ quadcopters in system $S$. The individual quadcopter's inertia matrix $\mathbf{J}_{q}$ has to be calculated experimentally. The position and orientation of each quadcopter in the system can vary, and so does the final inertia matrix of the system. The first step is to rotate $\vartheta_{i}^{o}$ the individual inertia matrices $\mathbf{J}_{q}$ for each quadcopter $i$ to obtained each individual rotated inertia matrices $\mathbf{J}_{i}$ :

$$
\mathbf{J}_{i}=\mathbf{R}_{z}\left(\vartheta_{i}^{o}\right) \cdot \mathbf{J}_{q} \cdot \mathbf{R}_{z}\left(\vartheta_{i}^{o}\right)^{T}
$$

In [41], the parallel axis theorem is used to calculate the inertia of a system of distributed quadcopters. This algorithm allows to transfer each individual $\mathbf{J}_{i}$ inertia matrix to the center of mass (CM) of system $S$. Using this method:

$$
\mathbf{J}_{C M}=\sum_{i=0}^{N}\left(\mathbf{J}_{i}+m_{q}\left(\left(\mathbf{P}_{i}^{o} \mathbf{P}_{i}^{o}\right) \mathbf{I}_{3 \times \mathbf{3}}-\mathbf{P}_{i}^{o}\left(\mathbf{P}_{i}^{o}\right)^{T}\right),\right.
$$

where $\mathbf{I}_{3 \times 3}$ is the $3 \times 3$ dimension identity matrix and $m_{q}$ the mass of a single quadcopter. However, these quadcopters are attached with each other using a rigid structure. Therefore, the last step is to add the inertia of this structure. Considering that the structure is composed 
by thin cylinders that connect the quadcopters in pairs, and that this cylinders can be defined by the length $l$ and radius $r$, the inertia matrix of each of these cylinders can be expressed as:

$$
\mathbf{J}_{\text {cyl }}=m_{\mathrm{cyl}}\left[\begin{array}{c}
\frac{3 r^{2}+l^{2}}{12} \\
\frac{3 r^{2}+l^{2}}{12} \\
\frac{r^{2}}{2}
\end{array}\right]
$$

In this case, the length $l$ can be expressed as the distance between two attached quadcopters $d_{i j}$, with $d_{i j}=d_{j i}$ and $d_{i i}=0$. This length $d_{i j}$ can be expressed as:

$$
d_{i j}=\left\|\left(\mathbf{P}_{i}^{o}-\mathbf{P}_{j}^{o}\right)\right\| .
$$

A binary vector with parameters $b_{i j}$ that is 1 only if the quadcopters $i$ and $j$ are, attached was included:

$$
b_{i j}=\left\{\begin{array}{l}
1 \text { if } i \text { and } j \text { attached } \\
0 \text { otherwise }
\end{array} .\right.
$$

Last, $\mathbf{P}_{i j}^{o}$ expresses the initial position of the connector $i j$ :

$$
\mathbf{P}_{i j}^{o}=\left(\left|\frac{\mathbf{P}_{i}^{o}}{2}\right|-\left|\frac{\mathbf{P}_{j}^{o}}{2}\right|\right) .
$$

Using the parallel axis theorem with the radius $r$ considered constant for every connection and the mass of each connector $i j$ expressed as $m_{c y l_{i j}}$ :

$$
\mathbf{J}_{\mathrm{cyl}}=\sum_{i=0}^{N-1} \sum_{j=0}^{N} b_{i j} m_{c y l_{i j}}\left(\left[\begin{array}{c}
\frac{3 r^{2}+d_{i j}^{2}}{12} \\
\frac{3 r^{2}+d_{i j}^{2}}{12} \\
\frac{r^{2}}{2}
\end{array}\right]+\left(\mathbf{P}_{i j}^{o} \mathbf{P}_{i j}^{o}\right) \mathbf{E}_{3}-\mathbf{P}_{i j}^{o}\left(\mathbf{P}_{i j}^{o}\right)^{T}\right) .
$$

The final inertia matrix of system $S$ is:

$$
\mathbf{J}_{S}=\mathbf{J}_{C M}+\mathbf{J}_{c y l} .
$$




\subsection{Integration of the Dynamical Model Equa- tions}

Using all the equations expressed in the previous pages, is obtained:

$$
\dot{\mathbf{s}}(k-1)=\left[\begin{array}{c}
\dot{x}(k-1) \\
\dot{y}(k-1) \\
\dot{z}(k-1) \\
\ddot{x}(k-1) \\
\ddot{y}(k-1) \\
\ddot{z}(k-1) \\
\dot{\phi}(k-1) \\
\dot{\theta}(k-1) \\
\dot{\psi}(k-1) \\
\dot{w}_{x}(k-1) \\
\dot{w}_{y}(k-1) \\
\dot{w}_{y}(k-1)
\end{array}\right],
$$

Using Eqs. 2.26 and 2.27, the expression of the full-state of the system $S$ in the current time step can be obtained:

$$
\mathbf{s}(k)=\left[\begin{array}{c}
x(k) \\
y(k) \\
z(k) \\
\dot{x}(k) \\
\dot{y}(k) \\
\dot{z}(k) \\
\phi(k) \\
\theta(k) \\
\psi(k) \\
w_{x}(k) \\
w_{y}(k) \\
w_{y}(k)
\end{array}\right] .
$$

\subsection{Noise and Bias}

All these equations assumed an ideal model of the system, in which possible biases or noises are not considered. However, this assump- 
tion is far from the reality, specially in the case of micro-quadcopters that due to its small size and weight, is characterized by their noisy measurements and motor gains. A simplified model of the noise and bias included in the real IMU's measurements is added to the model equations. More precisely, it is added to the estimation of the angular velocity in simulation, as it emulates the measurements obtained by the gyroscope. There is also noise added to the motors' PWM-thrust mapping done in simulation to model the variant value of the batteries charge, imperfect propellers or defects in the motors. These noises and biases need to be modeled empirically for each type of quadcopter to get an improved representation of the real system.

In the case of the measurements biases model, a unique bias value is obtained from a normal distribution for every quadcopter $i$, having:

$$
\mathbf{b}_{i} \sim \mathcal{N}\left(0, \sigma_{b}^{2}\right) .
$$

Evidently, the distance of the bias value to the origin will depend on the size of the chosen $\sigma_{b}$. Then, if the bias is added to the estimation of the angular velocity $\mathbf{w}_{i}$, for each quadcopter $i$ :

$$
\mathbf{w}_{i}^{\prime}(k)=\mathbf{w}_{i}(k)+\mathbf{b}_{i} .
$$

On the contrary, to emulate the real noise in the gyroscope of the IMU, a different value $\boldsymbol{\delta}_{i}(k)$ is obtained in each time step, from a normal distribution, with a variance of $\sigma_{\delta}^{2}$. Then, the estimated value of $\mathbf{w}_{i}^{\prime}$ is:

$$
\mathbf{w}_{i}^{\prime}(k)=\left(\mathbf{w}_{i}(k)+\mathbf{b}_{i}\right)\left(1+\boldsymbol{\delta}_{i}(k)\right) .
$$

The spread of this noise depends of the value of $\sigma_{\delta}$. Similarly, the motors' gain will be modified such that each of the motors in the system $S$ will have a different gain. This is done using, analog to the case of the bias in the angular velocities estimation, a value $g_{i j} \sim \mathcal{N}\left(0, \sigma_{b}^{2}\right)$ extracted from a normal distribution with variance $\sigma_{g}^{2}$, for each motor $i j$. Some noise $\gamma_{i j}(k)$ were also added to the motors, obtained from a normal distribution with variance $\sigma_{\gamma}^{2}$. The obtained rotation speed for each propeller $i j$ is:

$$
n_{i j}^{\prime}(k)=\left(n_{i j}(k)+g_{i j}\right)\left(1+\gamma_{i j}(k)\right)
$$




\section{Chapter 4}

\section{Estimation of the Physical Con- nection Parameters of Rigidly At- tached Quadcopters}

In Section 1.4 the main challenges of the present thesis are detailed. One of these challenges is the fact that the individual position $\mathbf{P}_{o_{i}}$ and rotation around the z-axis $\vartheta_{i}^{o}$ of each quadcopter are unknown and they can only be estimated using the individual measurements from the IMUs. The estimation of $\mathbf{P}_{i}^{o}$ can be obtained from the estimation of the distances between each pair of quadcopters $d_{i j}$, and the values of $\vartheta_{i}^{o}$, being the main parameters that need to be identified (See Fig. 4.1)

Solving this challenge implies that no a priori information will be required in order to achieve a stable flight for every possible configuration of $S$.

The described challenge presents two important difficulties. First, while the system $S^{\prime}$ s parameters are not identified, there is no manner to achieve stable flights. Thus, these parameters have to be identified before any flight attempt.

The second main difficulty is that, using only the IMUs can be understood as a dead reckoning process in which the estimation of the current state is computed from the state in the previous time step and, as a consequence, is subject to accumulative errors.

The objective of the method described in this section is to extract useful information using the IMU's gyroscope and accelerometer as the only sensors. As a consequence, only the linear acceleration and angular velocities can be measured. Fusing the measurements from 


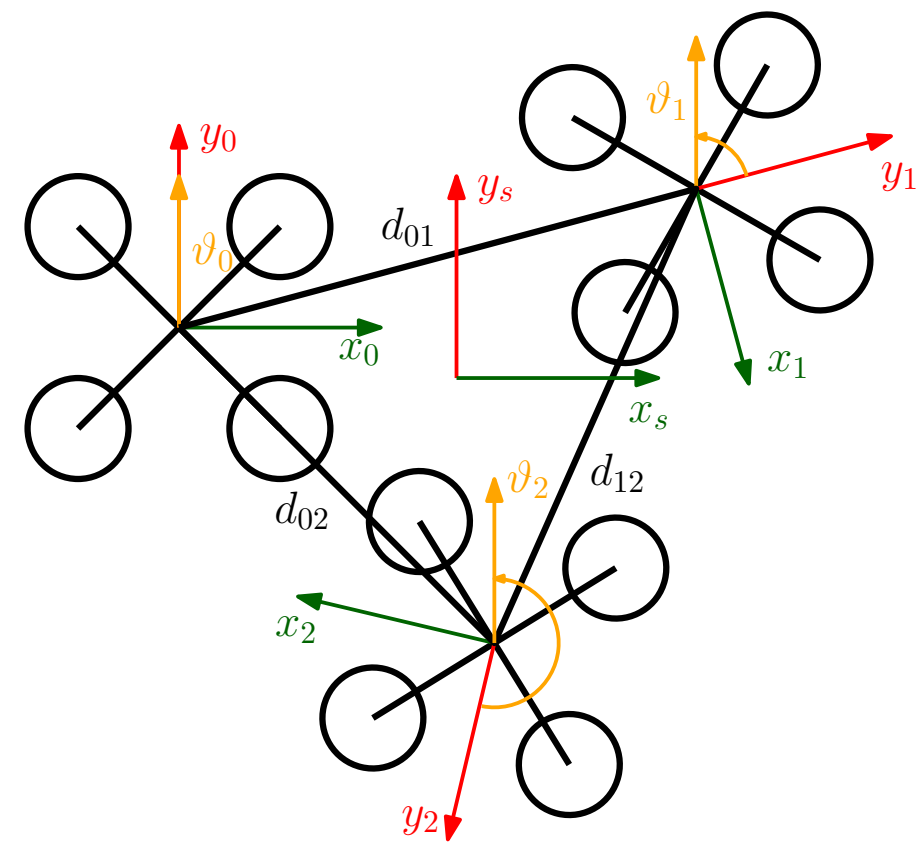

Figure 4.1: Simplified graphical representation of one possible configuration of the system.

both sensors can lead to noisy and biased estimation of the acceleration, attitude and angular velocities of each quadcopter. This method also implies to integrate the accelerations once to estimate the velocities and twice for the positions inducing the error drift in the positions estimation to increase rapidly. (See [36])

The general approach is summarized in Fig. 4.2 in which each color represents one of the main steps that have to be implemented. These steps will be explained in the following sections:

- Data Acquisition

- Preprocessing Strategy: Data Filtering

- Parameter Estimation 


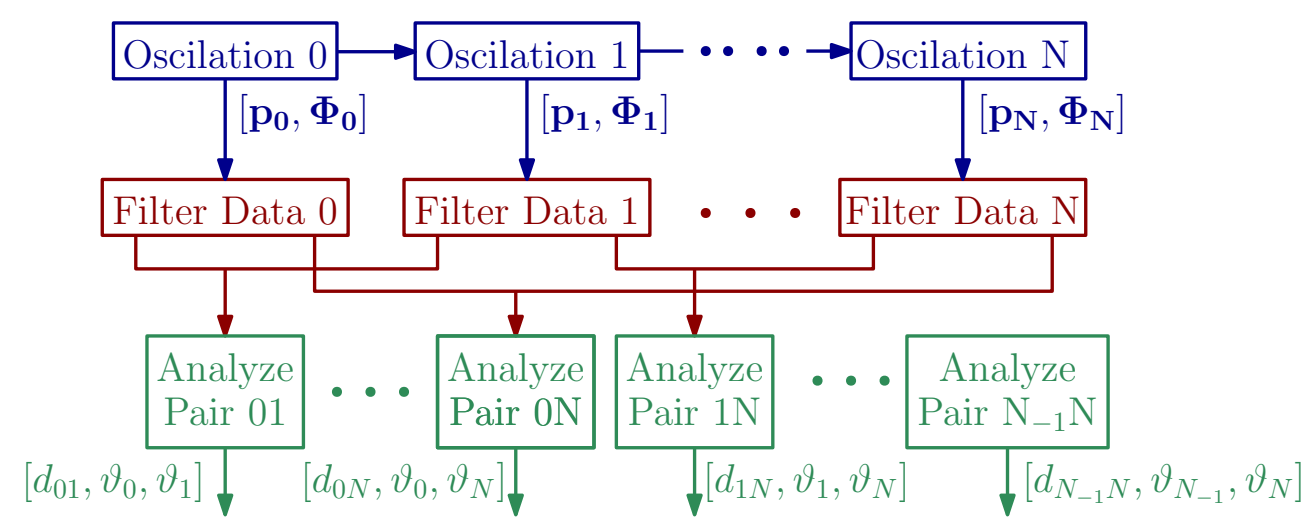

Figure 4.2: Simplified graphical representation of the general approach for the parameter estimation step.

\subsection{Data Acquisition and Preprocessing Strat- egy}

The system needs to be forced to have some variations in its measurements in order to get valuable data. However, the system is not able to perform any stable flight before the unknown parameters are estimated. The proposed solution is application of dynamical oscillations to the system from the ground in order to obtain useful data from the sensors. The idea is that the system lifts only a couple of centimeters from the ground during a short period of time $(<0.2 \mathrm{~s})$ with the aim of obtaining useful data.

\subsubsection{Dynamic Oscillations}

To obtain the described oscillations, the on-board quadcopter's PID controllers will be used. The attitude states $\phi, \theta$, angular velocity around the z-axis $w_{z}$ and height $z$ controllers combined will be used, as shown in Fig. 4.3 .

The strategy is designed to be repeated as many times as number $N$ of quadcopters in the system $S$. In each iteration a different quadcopter will be chosen as the leader $L$, to use a distinct set-point vector $S P_{L}=\left[\phi_{L}, \theta_{L}, w_{z_{L}}, z_{L}\right]$ while the rest of the quadcopters (slaves $s l$ ) will use another set-point vector $S P_{s l}=\left[\phi_{s l}, \theta_{s l}, w_{z_{s l}}, z_{s l}\right]$. Then the leader $L$ and slave $s l$ set-points values in one iteration can be defined as: 


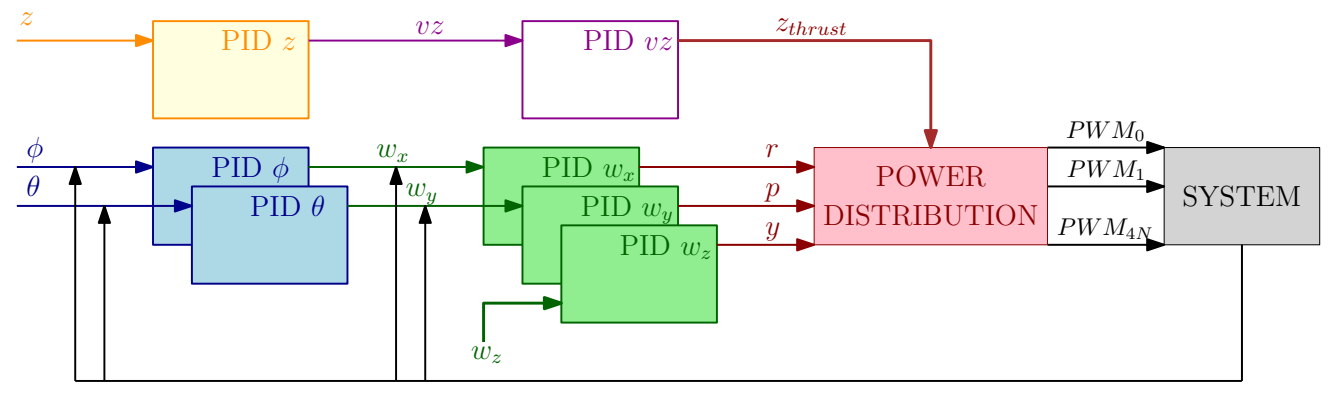

Figure 4.3: Graphical representation of the structure of the structure of the on-board quadcopter's controller used in the Parameter Estimation step.

$$
\begin{gathered}
S P_{L}= \begin{cases}{\left[\phi_{L}, \theta_{L}, w_{z_{L}}, z_{L}\right]} & \text { if } t \leq t_{o s c} \mathrm{~s} \\
{[0,0,0,0]} & \text { if } t_{o s c} \leq t \leq t_{i t} \mathrm{~s}\end{cases} \\
S P_{s l}=\left\{\begin{array}{ll}
{\left[\phi_{s l}, \theta_{s l}, w_{z_{s l}}, z_{s l}\right]} & \text { if } t \leq t_{o s c} \mathrm{~s} \\
{[0,0,0,0]} & \text { if } t_{o s c} \leq t \leq t_{i t} \mathrm{~s}
\end{array} .\right.
\end{gathered}
$$

with $t_{\text {osc }}$ the arbitrary time that the system will lift from the ground in each oscillation. The objective of this process is to obtain significant variations in the measurements of the gyroscope and accelerometer in each of the axis. For this reason, very extreme values of the desired roll $\phi$, pitch $\theta$ and $z$-axis angular velocity were chosen along with very different desired heights between the leader $L$ and the slaves $s l$. These heights were chosen to not lift the quadcopters excessively from the ground to ensure that they are not damaged. The next set-points vectors were chosen :

$$
\begin{aligned}
& {\left[\phi_{L}, \theta_{L}, w_{z_{L}}, z_{L}\right]=[0 \mathrm{deg}, 0 \mathrm{deg}, 0 \mathrm{deg} / \mathrm{s}, 0.30 \mathrm{~m}]} \\
& {\left[\phi_{q}, \theta_{q}, w_{z_{q}}, z_{q}\right]=[20 \mathrm{deg}, 20 \mathrm{deg}, 250 \mathrm{deg} / \mathrm{s}, 0.05 \mathrm{~m}] .}
\end{aligned}
$$

With $N$ being the number of quadcopters, the final algorithm is described in Alg. 1.

\subsection{Data Filtering}

For the parameter estimation step, no external measurements are received, only the ones obtained by the IMU, and therefore, the full-state 


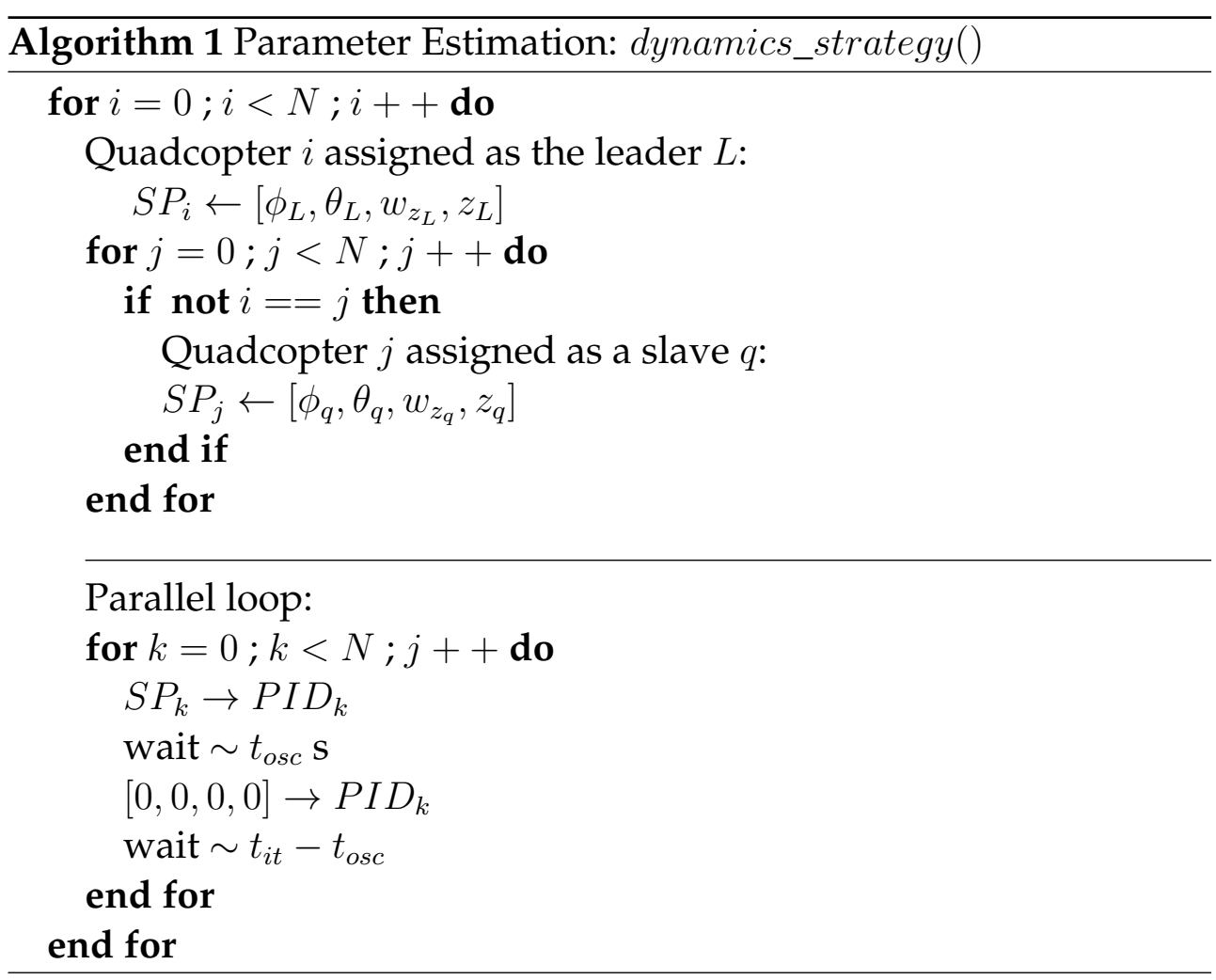

estimation drifts rapidly. For this reason, it was implemented an improved full-state estimator that will be used only for this step. This custom estimator is able to reduce the drift increment speed considerably allowing to obtain useful measurements from the individual IMUs. Generally, most of the commercial quadcopters use a Kalman filter for the estimation of the full-state. In the case of this thesis, the already built-in Kalman filter of each quadcopter will be used when the system of quadcopters is flying. Only some modifications in the $\mathbf{B}_{k}$ and $\mathbf{H}_{k}$ matrices will be included to adapt the system model to the estimated parameters.

The quaternions $\left[q_{0}, q_{1}, q_{2}, q_{3}\right]$ are estimated using a sensor fusion step, based on Mahony's work on complementary filters [47], that merges the accelerometer's and gyroscope's measurements to get a better estimation of the attitude. Then, the rotation matrix $\mathbf{R}$ equa- 
tions can be written in terms of the estimated quaternion:

$$
\begin{aligned}
& R_{00}=q_{0}^{2}+q_{1}^{2}-q_{2}^{2}-q_{3}^{2} \\
& R_{01}=2 q_{1} q_{2}-2 q_{0} q_{3} \\
& R_{02}=2 q_{1} q_{3}+2 q_{0} q_{2} \\
& R_{10}=2 q_{1} q_{2}+2 q_{0} q_{3} \\
& R_{11}=q_{0}^{2}-q_{1}^{2}+q_{2}^{2}-q_{3}^{2} . \\
& R_{12}=2 q_{2} q_{3}-2 q_{0} q_{1} \\
& R_{20}=2 q_{1} q_{3}-2 q_{0} q_{2} \\
& R_{21}=2 q_{2} q_{3}+2 q_{0} q_{1} \\
& R_{22}=q_{0}^{2}-q_{1}^{2}-q_{2}^{2}+q_{3}^{2} .
\end{aligned}
$$

The simplified model of the quadcopter can be written:

$$
\begin{aligned}
& x(k)=x(k-1)+\dot{x}(k) \Delta k \\
& y(k)=y(k-1)+\dot{y}(k) \Delta k \\
& z(k)=z(k-1)+\dot{z}(k) \Delta k \\
& \dot{x}(k)=\dot{x}(k-1)+R_{00} \cdot \operatorname{acc}_{x_{t}} \Delta k+R_{01} \cdot \operatorname{acc}_{y_{t}} \Delta k+R_{02} \cdot \operatorname{acc}_{z_{t}} \Delta k \\
& \dot{y}(k)=\dot{y}(k-1)+R_{10} \cdot \operatorname{acc}_{x_{t}} \Delta k+R_{11} \cdot \operatorname{acc}_{y_{t}} \Delta k+R_{12} \cdot \operatorname{acc}_{z_{t}} \Delta k \\
& \dot{z}(k)=\dot{z}(k-1)+R_{20} \cdot \operatorname{acc}_{x_{t}} \Delta k+R_{21} \cdot \operatorname{acc}_{y_{t}} \Delta k+R_{22} \operatorname{acc}_{z_{t}} \Delta k .
\end{aligned}
$$

However, these equations themselves lead to an estimate of the full-state that drifts rapidly. The main reason for this drift is that this simplified model is not able to detect if the quadcopter has stopped or is moving as the only information that can be read is the acceleration, and zero acceleration only means null or constant velocity. It is necessary to add a method to the Eq. 4.6 that estimates whether the quadcopter has stopped or not. Fig. 4.4 represents the evolution the simulation of the drift when a quadcopter is lifted for a short period and return back to the same initial position.

In [57] a novel method in which the use the short-term variance of the measured acceleration to determine if a system has zero velocity or not is proposed. Based on this idea, a very simple method that uses the variance to determine if a quadcopter is static or flying is developed. For every new prediction of the full-state, the variance of the last $K$ measurements of the acceleration is computed. This way, a threshold of this variance from which it can be considered that the quadcopter 


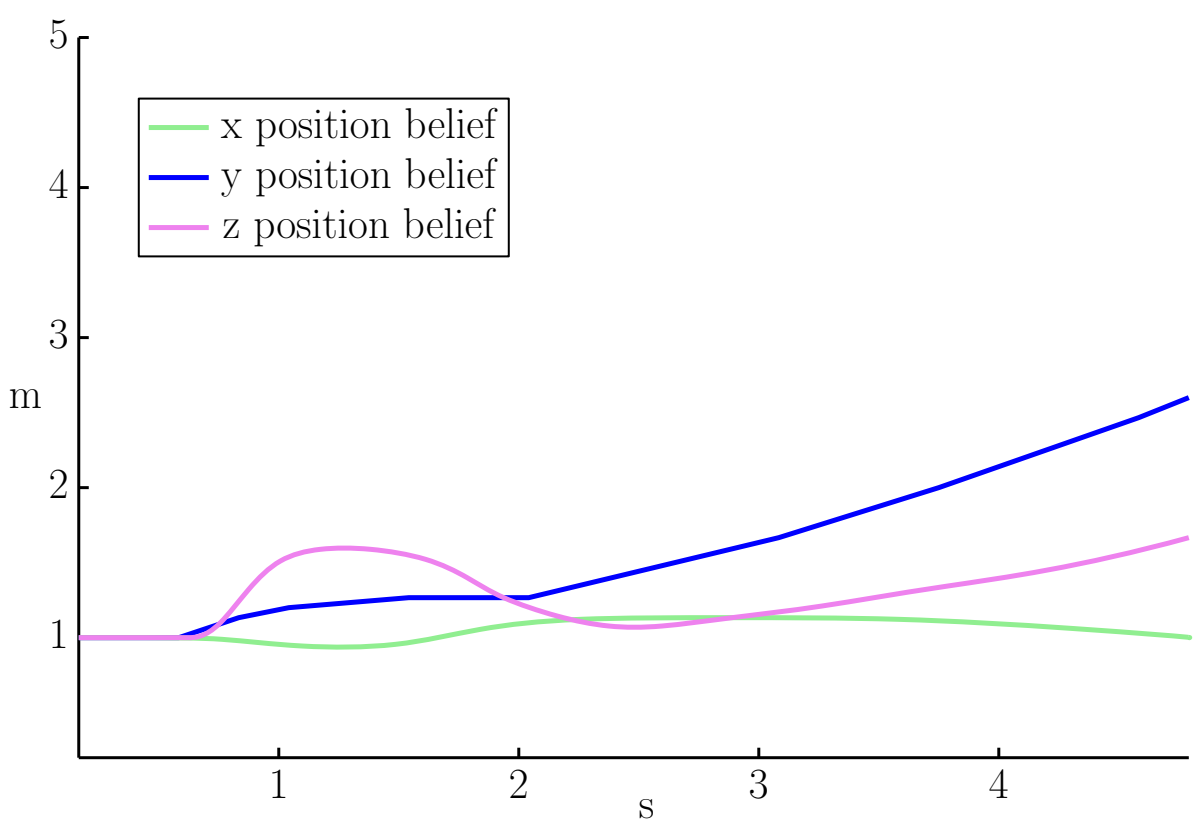

Figure 4.4: Evolution of the estimation of the $x, y$ and $z$ positions using a Kalman filter without any external measurements after a quadcopter is lifted and left back in the ground in the same position.

has started moving can be experimentally determined. If the calculated variance is lower than this threshold all the velocities are set to zero in the Eq. 4.6. The approach is shown with more detail in Alg. 2.

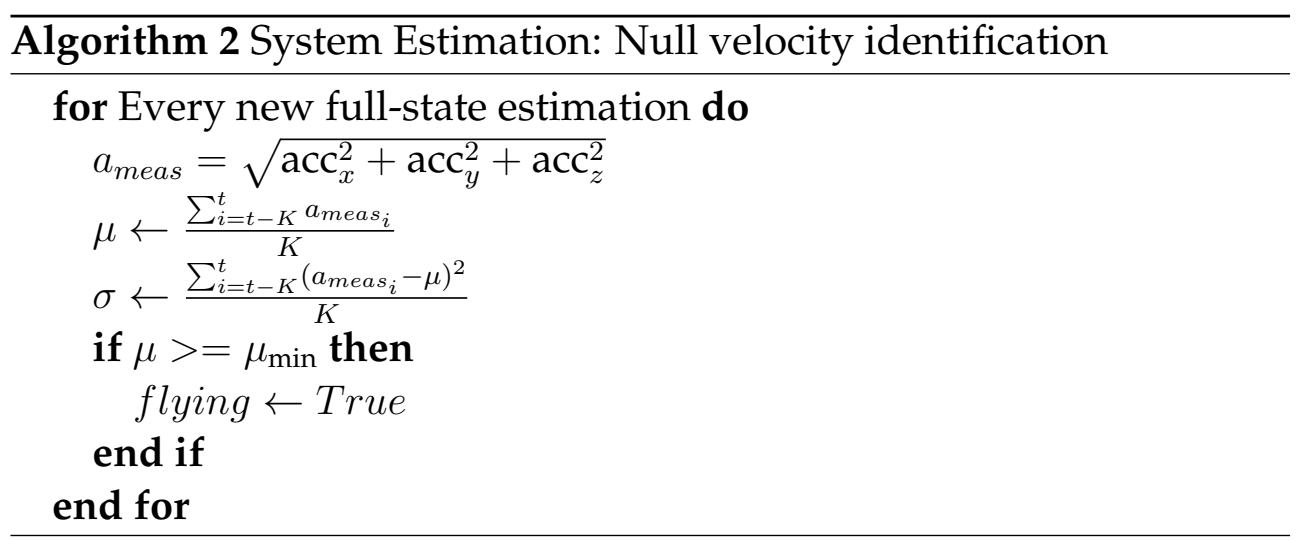

With the application of this simple method, the estimation of the position was improved as it is shown in Fig. 4.5. Even though the estimator is now able to detect whether the quadcopter has stopped or not it can be observed that the $\mathrm{z}$ position estimation never goes back to zero when it should. This is due to the fact that the measurements 


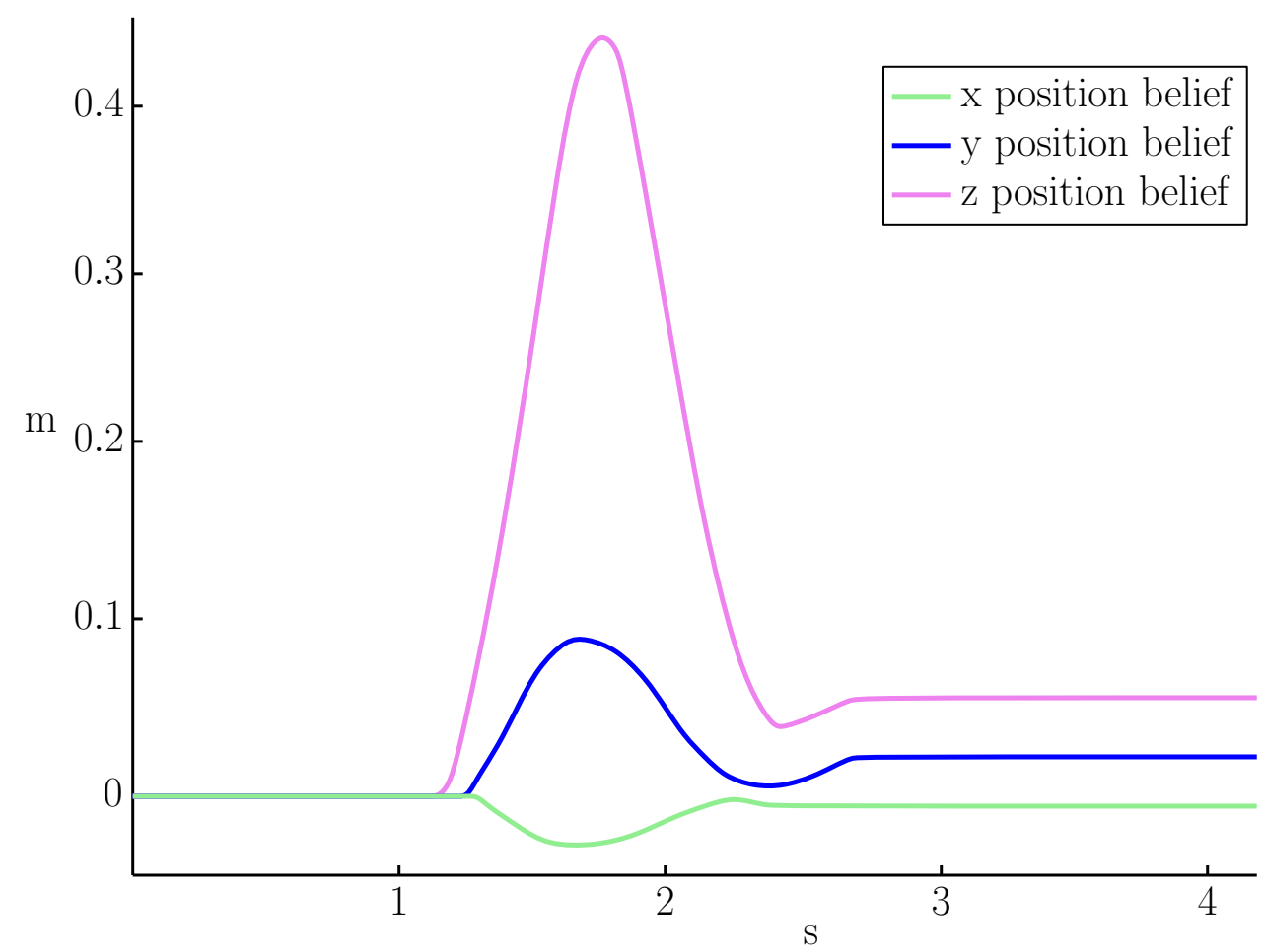

Figure 4.5: Estimation of the $x, y$ and $z$ positions using the described approach to predict if a quadcopter is moving or static after it is is lifted and left back in the ground in the same position.

from the accelerometer are not perfect and then, the acceleration and deceleration curves do not enclose exactly the same area.

The solution chosen was to use only the acceleration curve as the drift will be smaller than in the deceleration curve case. For every iteration in Alg. 1, it will only be stored and used the data from the initial time-step of the iteration until the leader quadcopter $L$ reaches its maximum height point. The rest of the data will be discarded. Also, before every iteration of Alg. 1 the estimators are restarted in order to reset the accumulative error or drift and get cleaner data. In Fig. 4.6 it is shown the final appearance of the estimation of $\left[x_{i}, y_{i}, z_{i}, \phi_{i}, \alpha_{i}, \psi_{i}\right]$ for each quadcopter $i$ in the case of 2 attached quadcopters.

\subsubsection{Data Interpolation}

The last issue that need to be solved is that the quadcopters are not synchronized and their measurements are not taken at the exact same 

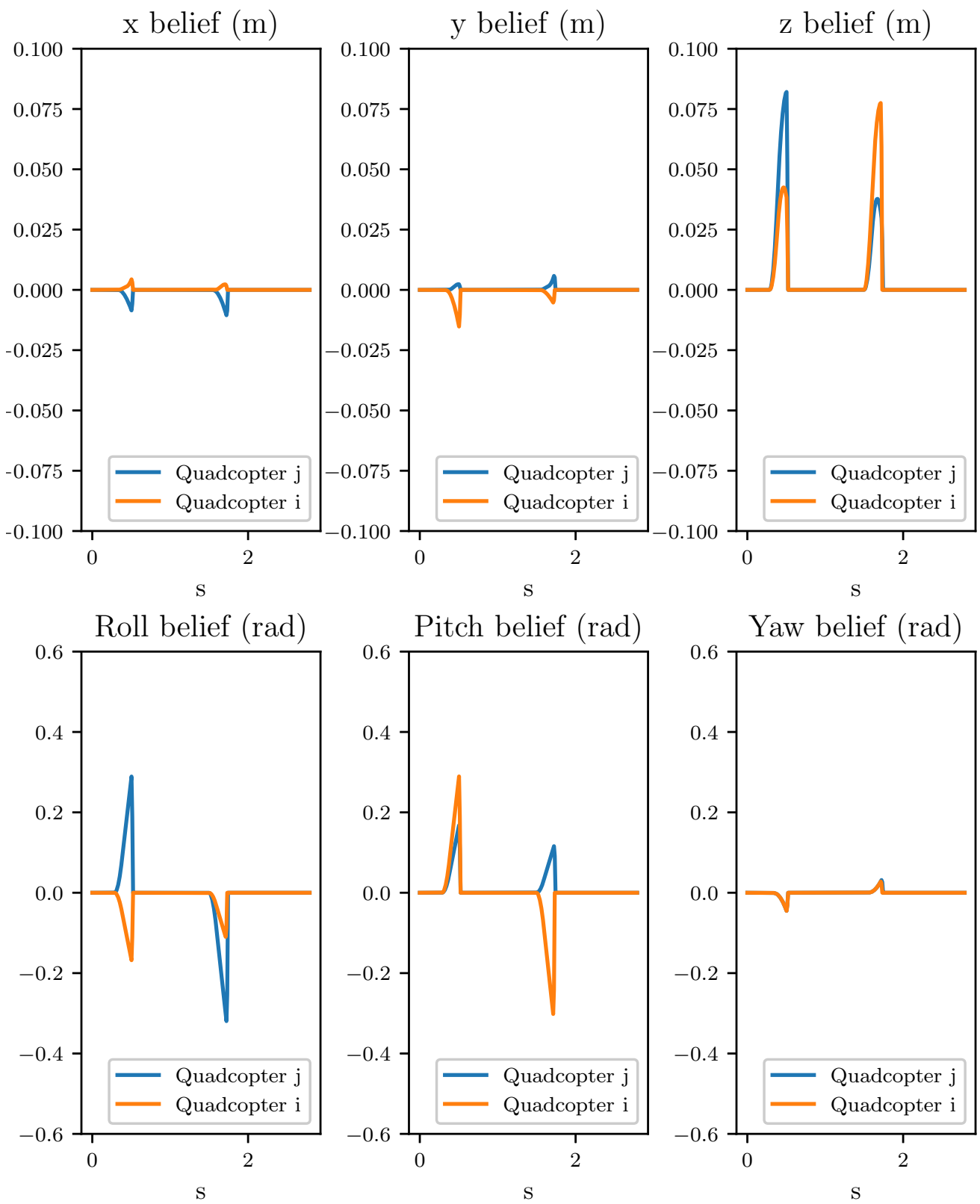

Figure 4.6: Estimation of $[x, y, z, \phi, \alpha, \psi]$ for each quadcopter in the pair $i j$.

time step. This would not allow to perform a correct estimation of the parameters as it is necessary to use measurements that were taken in 


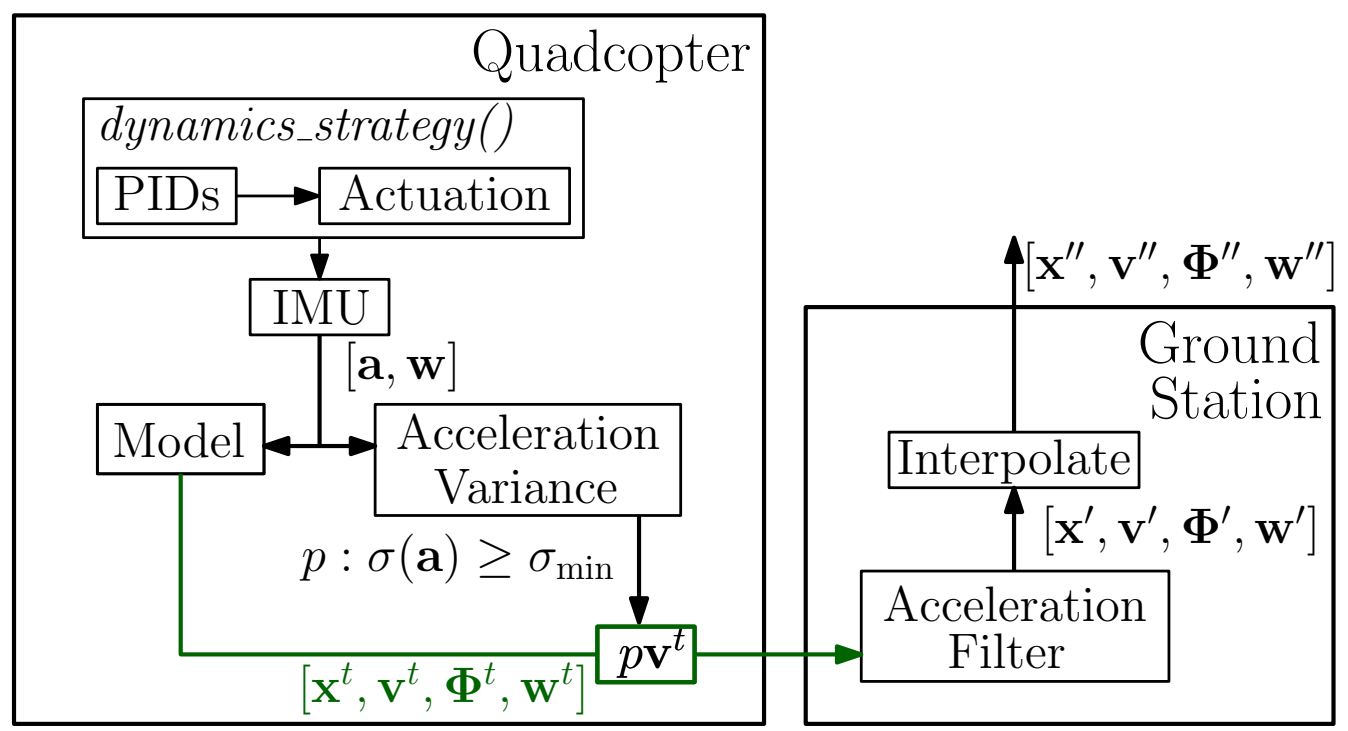

Figure 4.7: Schematic data acquisition and preprocessing approach.

the exact same instant. To solve this, the obtained data is linearly interpolated so a continuous representation of the discrete measurements can be approximated. This can be performed as the data acquisition rate is very high $(500 \mathrm{~Hz})$. As a consequence, the distance between consecutive points is small enough that no data loss will be produced.

The entire data acquisition process is shown in Fig. 4.7.Using this strategy, it is feasible to extract relevant information from each of the IMUs.

\subsection{Parameter Estimation}

The estimation of $\vartheta_{i}$ and $\mathbf{P}_{i}$ can be understood as an iterative algorithm in which these parameters are calculated for each pair of quadcopters in $S$ by analyzing one pair per iteration step. The idea is to first execute the steps described in Alg. 1 and gather all the needed data.

Using this data, these parameters are estimated for each possible pair $i j$ of quadcopters in the system $S$. Once each $d_{i j}$ and $\vartheta_{i}$ has been calculated, the position of each quadcopter $i$ is completely defined relative to an arbitrarily chosen coordinate frame for the system $S$. Subsequently, the last step would be to estimate the initial position $P_{o_{i}}$ of each quadcopter relative to the system $S$ coordinate frame using these estimated parameters. 


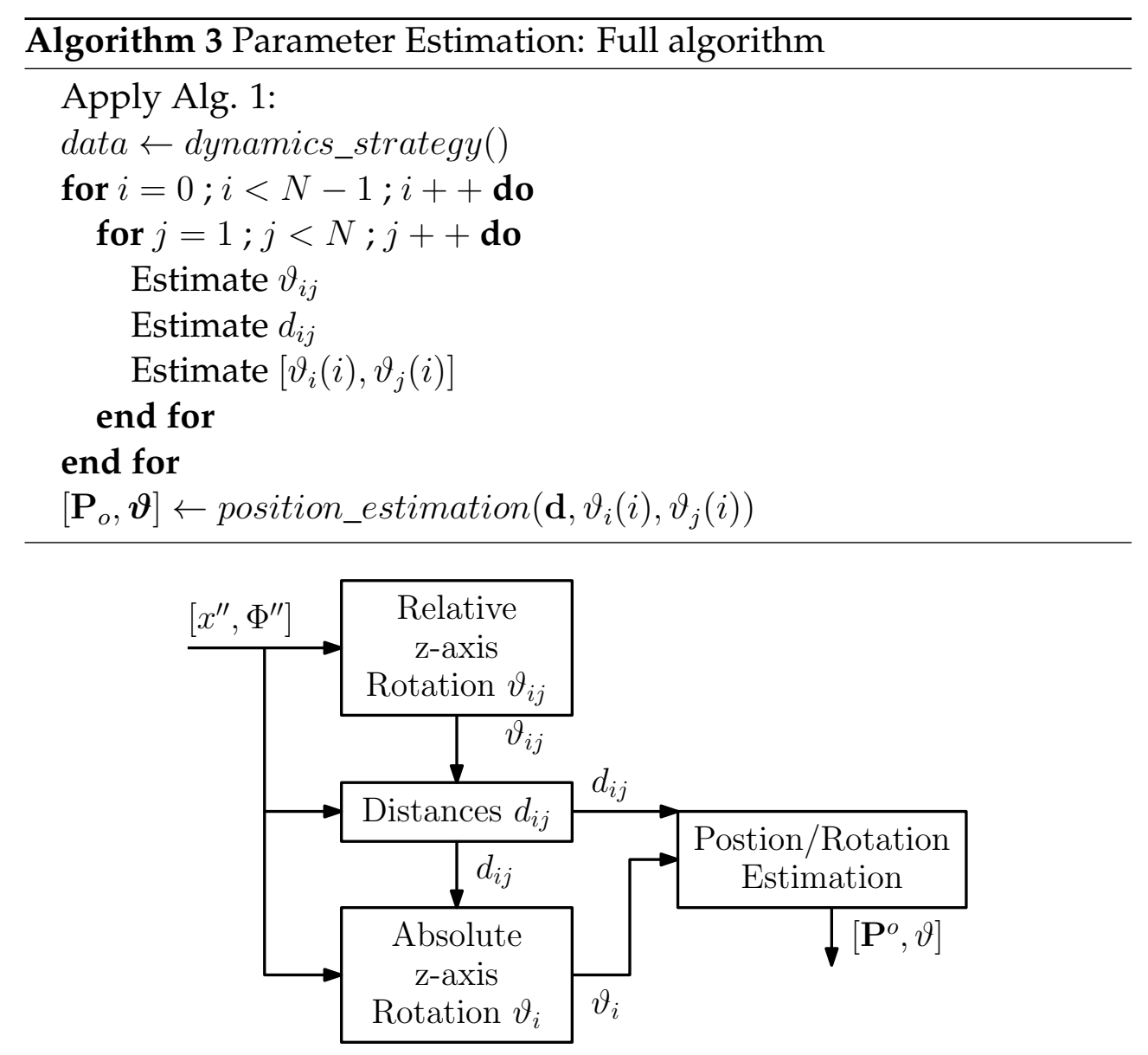

Figure 4.8: Schematic parameter estimation approach.

The general process is depicted in Fig. 4.8. These main steps will be described in the following subsections:

- Relative $\mathrm{z}$-axis Rotation: the relative rotation around the $\mathrm{z}$-axis $\vartheta_{i j}$ between each possible pair of quadcopters is estimated.

- Distances: using the relative rotations and the gathered and filtered data, the distances between each pair of quadcopters $d_{i j}$ is estimated.

- Absolute z-axis Rotation: using both the relative z-axis rotation and distances between each pair of quadcopters, the absolute rotation around the z-axis $\vartheta_{i}$ is estimated for each quadcopter, relative to the arbitrary coordinate frame of the system. 


\subsubsection{Relative z-axis Rotation}

The first parameter that can be estimated from the measurements is the initial relative rotation around the z-axis $\vartheta_{i j}$ between each pair of attached quadcopter, that has the following properties:

$$
\left\{\begin{array}{l}
\vartheta_{i j}=0 \\
\vartheta_{i j}=-\vartheta_{j i}
\end{array} \quad \text { if } i=j .\right.
$$

Lemma 4.3.1. The relative rotation between each pair of quadcopters ij can be calculated as:

$$
\vartheta_{i j}=\frac{\sum_{t=0}^{T} \operatorname{atan}\left(\frac{\theta_{i}^{t}}{\phi_{i}^{t}}\right)-\operatorname{atan}\left(\frac{\theta_{j}^{t}}{\phi_{j}^{t}}\right)}{T} .
$$

Proof. For both quadcopters $i j$, the coordinate system will be considered in terms of roll and pitch $(\phi, \theta)$. For each time-step $t$, the vector $\left[\begin{array}{ll}\phi_{i}^{t} & \theta_{i}^{t}\end{array}\right]$ will be measured. Both quadcopters are situated along the same plane and the connection is supposed to be perfectly rigid. As a consequence, both quadcopters will experiment the same amount of attitude in each time step. The idea is simple, two rigidly attached objects will experiment the exact same amount of motion. As both quadcopters are constrained to be situated along the same plane they will experiment the same attitude variation in each time-step. Then, it can be assumed that the module of each pair $\left[\begin{array}{ll}\phi_{i}^{t} & \theta_{i}^{t}\end{array}\right]$ satisfies:

$$
\left\|\left[\begin{array}{ll}
\phi_{i}^{t} & \theta_{i}^{t}
\end{array}\right]\right\|=\left\|\left[\begin{array}{ll}
\phi_{j}^{t} & \theta_{j}^{t}
\end{array}\right]\right\|=T .
$$

If a circle with radius $T$ is defined in the coordinate system of $(\phi, \theta)$, the measured values of $\left[\begin{array}{ll}\phi_{i}^{t} & \theta_{i}^{t}\end{array}\right]$ and $\left[\begin{array}{ll}\phi_{i}^{t} & \theta_{i}^{t}\end{array}\right]$ can be represented as in Fig. 4.9.

Then is direct to get the expression of any possible angle $\alpha_{i}$ :

$$
\left.\begin{array}{l}
T \sin \left(\alpha_{i}\right)=\theta_{i} \\
T \cos \left(\alpha_{i}\right)=\phi_{i}
\end{array}\right\} \Rightarrow \alpha_{i}=\operatorname{atan}\left(\frac{\theta_{i}}{\phi_{i}}\right) .
$$

For every time step $t$, the relative angle $\vartheta_{i j}^{t}$ between both quadcopters will be:

$$
\hat{\vartheta}_{i j}^{t}=\alpha_{i}^{t}-\alpha_{j}^{t}=\operatorname{atan}\left(\frac{\theta_{i}^{t}}{\phi_{i}^{t}}\right)-\operatorname{atan}\left(\frac{\theta_{j}^{t}}{\phi_{j}^{t}}\right) .
$$




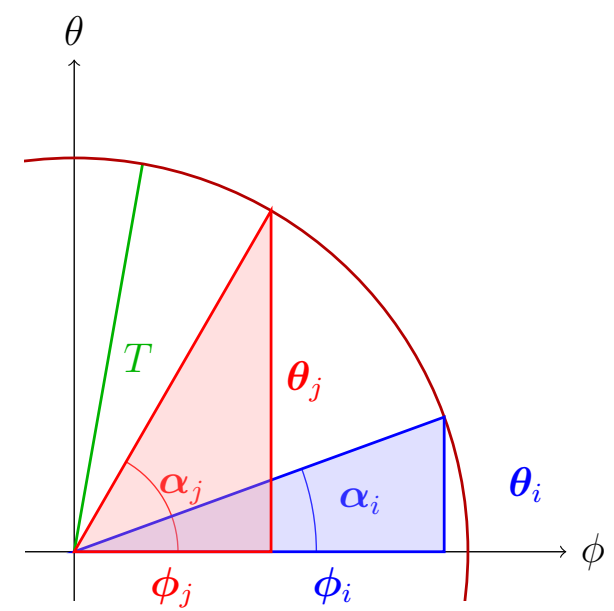

Figure 4.9: Representation of the vectors $\left[\phi_{i}^{t} \theta_{i}^{t}\right]^{T}$ and $\left[\phi_{j}^{t} \theta_{j}^{t}\right]^{T}$ along with the angles $\alpha_{i}$ and $\alpha_{j}$ between this vectors and the $\phi$ axis

This calculated $\hat{\vartheta}_{i j}^{t}$ should be the same in every time step. However, the measurements are noisy and due to this unmodeled noise, the estimation is also. Therefore, all the measurements taken during the application of Alg. 1 will be used to calculate $\hat{\vartheta}_{i j}^{t}$ for each time-step $t$ and the final value of $\vartheta_{i j}$ is calculated using the mean value of these estimations:

$$
\vartheta_{i j}=\frac{\sum_{t=0}^{T} \hat{\vartheta}_{i j}^{t}}{T}
$$

\subsubsection{Distances}

The next step in the paramter estimation method is to calculate the distances $d_{i j}$ between each pair of quadcopters $i j$.

Lemma 4.3.2. Assume the following equation of a line:

$$
n_{x}\left(x-\left(x_{i}-\tilde{x}_{j}\right)\right)+n_{y}\left(y-\left(y_{i}-\tilde{y}_{j}\right)\right)-n_{z}\left(\left(z_{i}-\tilde{z}_{j}\right)\right)=0,
$$

where $\mathbf{n}$ is defined as:

$$
\mathbf{n}=\frac{\mathbf{R}_{x}\left(\phi_{i}\right) \mathbf{R}_{y}\left(\theta_{i}\right) \mathbf{R}_{z}\left(\psi_{i}\right)\left[\begin{array}{lll}
0 & 0 & 1
\end{array}\right]^{T}}{\left\|\mathbf{R}_{x}\left(\phi_{i}\right) \mathbf{R}_{y}\left(\theta_{i}\right) \mathbf{R}_{z}\left(\psi_{i}\right)\left[\begin{array}{lll}
0 & 0 & 1
\end{array}\right]^{T}\right\|}
$$


and $\left[\begin{array}{lll}\tilde{x}_{j} & \tilde{y}_{j} & \tilde{z}_{j}\end{array}\right]^{T}$ are obtained by applying:

$$
\left[\begin{array}{lll}
\tilde{x}_{j} & \tilde{y}_{j} & \tilde{z}_{j}
\end{array}\right]=\mathbf{R}_{z}\left(\vartheta_{i j}\right)\left[\begin{array}{lll}
x_{j} & y_{j} & z_{j}
\end{array}\right]^{T} .
$$

Let the intersection point of all the lines calculated for each time step using Eq. 4.13 be defined as:

$$
P_{\text {int }}=\left[\begin{array}{ll}
x_{i n t} & x_{i n t}
\end{array}\right] .
$$

Then the distance between each pair of quadcopters ij can be expressed as the distance between this intersection point $P_{\text {int }}$ and the origin:

$$
d_{i j}=\left\|\left(x_{i n t}, y_{i n t}\right)\right\| .
$$

Proof. A virtual coordinate frame that is aligned with the quadcopter's $i$ coordinate frame will be used being the origins of both the virtual system's $O_{\text {virtual }}$ and quadcopter's $O_{i}$ coincident:

$$
O_{\text {virtual }}=O_{i}
$$

The equation of the plane defined by both quadcopters $i$ and $j$ can be calculated using the measurements from their IMUs. The normal vector of this plane can be obtained using the estimated attitude of one of the quadcopters from the pair $i j$, the quadcopter $i$. The normal vector of the plane is expressed as:

$$
\mathbf{n}=\frac{\mathbf{R}_{x}\left(\phi_{i}\right) \mathbf{R}_{y}\left(\theta_{i}\right) \mathbf{R}_{z}\left(\psi_{i}\right)\left[\begin{array}{lll}
0 & 0 & 1
\end{array}\right]^{T}}{\left\|\mathbf{R}_{x}\left(\phi_{i}\right) \mathbf{R}_{y}\left(\theta_{i}\right) \mathbf{R}_{z}\left(\psi_{i}\right)\left[\begin{array}{lll}
0 & 0 & 1
\end{array}\right]^{T}\right\|},
$$

with $\mathbf{R}_{x}, \mathbf{R}_{y}$ and $\mathbf{R}_{z}$ expressing the rotation matrices around the $\mathbf{x}$, $\mathrm{y}$ and $\mathrm{z}$-axes respectively (See Eq. 2.4). With this normal vector of the plane, it is now only necessary a point of the plane to obtaine its equations. As the position of the quadcopters $i$ is measured in this new virtual coordinate frame, and the quadcopter $i$ is situated over this plane, a point of the plane is $\left[\begin{array}{lll}P_{0} & P_{1} & P_{2}\end{array}\right]=\left[\begin{array}{lll}x_{i} & y_{i} & z_{i}\end{array}\right]$. Then, the final equation of the plane in the virtual coordinate frame is:

$$
n_{x}\left(x-x_{i}\right)+n_{y}\left(y-y_{i}\right)+n_{z}\left(z-z_{i}\right)=0 .
$$




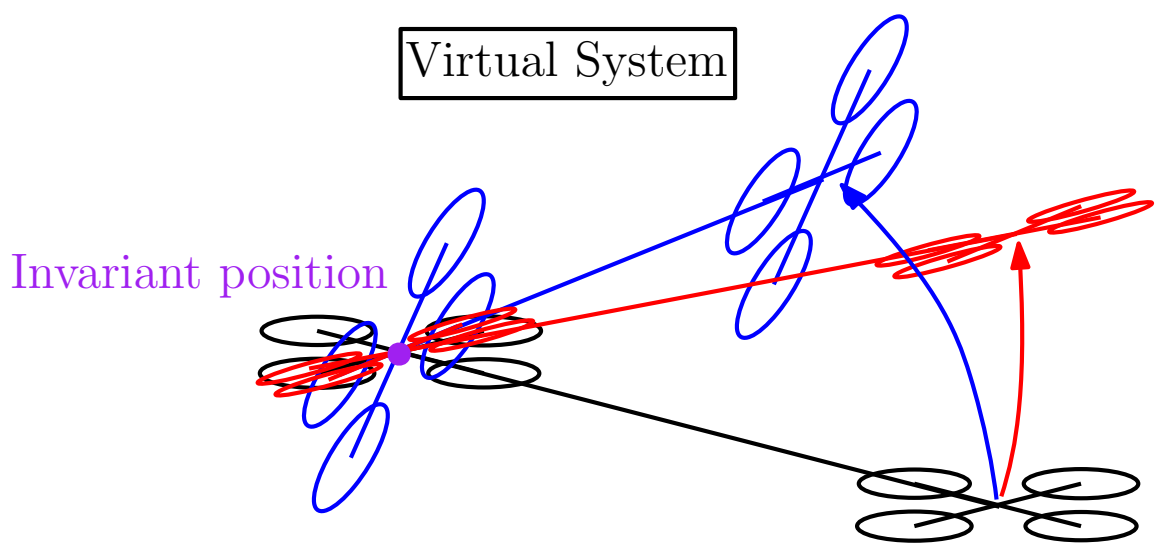

Figure 4.10: Two different oscillations represented in the defined virtual system.

As the relative angle between both quadcopters $\vartheta_{i j}$ is known, then the coordinate system of the quadcopter $j$ can be rotated so that its state estimation can be used in the same coordinate frame as in $i$. The new coordinate frame of quadcopter $j$ is:

$$
O X Y Z_{\text {virtual }}=O X Y Z_{i}=R_{z}\left(\vartheta_{i j}\right) O X Y Z_{j}=O \widetilde{X Y} Z_{j}
$$

and the new state of quadcopter $j$ is expressed:

$$
\left[\tilde{x}_{j}, \tilde{y}_{j}, \tilde{z}_{j}, \dot{\tilde{x}}_{j}, \dot{\tilde{y}}_{j}, \dot{\tilde{z}}_{j}, \tilde{\phi}_{j}, \tilde{\theta}_{j}, \tilde{\psi}_{j}, \tilde{w_{x_{j}}}, \tilde{w_{y_{j}}},{\tilde{w_{z}}}_{j}\right] .
$$

Now the position of quadcopter $j$ in the virtual coordinates frame is subtracted to the position of $i$ to get a virtual position of the system in which quadcopter $j$ remains virtually in the same position for each measurement or time step, see Fig. 4.10. The new plane defined in terms of the virtual coordinate frame is:

$$
n_{x}\left(x-\left(x_{i}-\tilde{x}_{j}\right)\right)+n_{y}\left(y-\left(y_{i}-\tilde{y}_{j}\right)\right)+n_{z}\left(z-\left(z_{i}-\tilde{z}_{j}\right)\right)=0 .
$$

If the intersection line between the plane defined by this new virtual system and the plane $z=0$ is calculated then it is obtained a line that passes through the virtual position of $j^{\prime}$ in the virtual coordinate frame. This line is expressed as:

$$
n_{x}\left(x-\left(x_{i}-\tilde{x}_{j}\right)\right)+n_{y}\left(y-\left(y_{i}-\tilde{y}_{j}\right)\right)-n_{z}\left(\left(z_{i}-\tilde{z}_{j}\right)\right)=0 .
$$

Calculating this line for different time steps, it can be obtained an intersection point $\left[\begin{array}{ll}x_{\text {int }} & y_{\text {int }}\end{array}\right]$ that will coincide with the virtual position of 


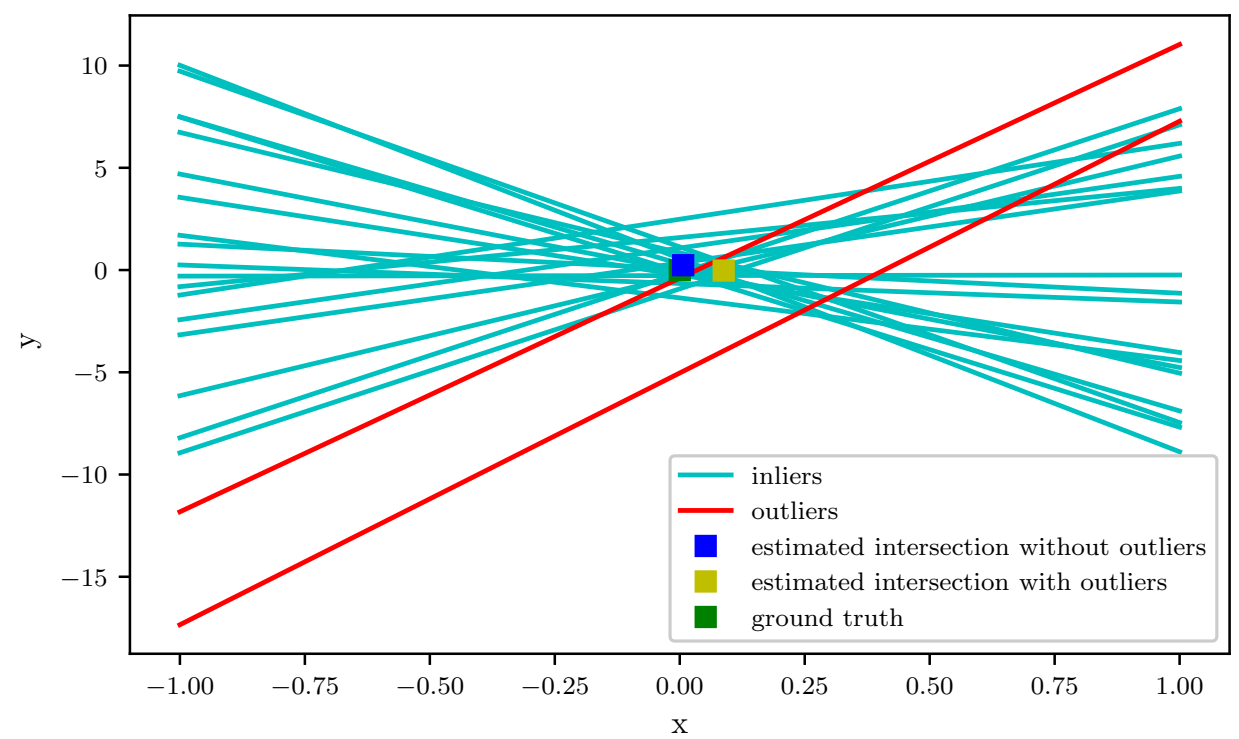

Figure 4.11: Plot of 20 lines intersecting around the origin. This plot was created by computing 20 lines that intersect in the origin and adding to each of them random white noise. The variance of the noise used for the red lines is 4 times bigger than the one used for the blue lines in order to obtain the outliers.

quadcopter $j$ in the virtual coordinate frame. As quadcopter $i$ 's origin $O_{i}$ is considered to coincide with the virtual coordinate frame's origin $O_{\text {virtual }}$, the distance between quadcopter $i$ and quadcopter $j$ will be:

$$
d_{i j}=\left\|\left(x_{\text {int }}, y_{\text {int }}\right)\right\| \text {. }
$$

The estimation of the position for both quadcopters $i$ and $j$ is calculated from the IMUs' measurements. The errors in these measurements will produce deviation in the intersection lines, producing a set of intersection points around the real one. One option could be to use a least squares algorithm to get the a good approximation of the true intersection point (See [70]).

However, as it can be observed in Fig. 4.11, the main problem with these algorithm is that it is outliers-sensitive. Only 2 outliers in the example set (in red) used for Fig. 4.11 produce an increasing deviation in the estimated intersection point. Therefore, any possible error in the 
estimation of the lines will lead to an incorrect estimation of this point. Least squares alone can not be considered as the optimal method for this step.

Lemma 4.3.3. Let the equation of a line in terms of its slope $m$ and intercept $c$ :

$$
y=m x+c .
$$

The pairs $\left[\begin{array}{ll}m^{k} & c^{k}\end{array}\right]$ of a set of $K$ lines that intersect in the same point represent a perfect line in the $\left[\begin{array}{ll}m & c\end{array}\right]$ coordinate frame.

Proof. In the case of the line defined by Eq. 4.25, its values of the slope and intercept can be expressed as:

$$
\begin{gathered}
m=\frac{n_{x}}{n_{y}}\left(x_{i}-\tilde{x}_{j}\right)+\left(y_{i}-\tilde{y}_{j}\right)+\frac{n_{z}}{n_{y}}\left(z_{i}-\tilde{z}_{j}\right) \\
c=-\frac{n_{x}}{n_{y}} .
\end{gathered}
$$

Then it can be defined a value of $\left[\begin{array}{ll}m^{k} & c^{k}\end{array}\right]$ for every measurement $K$. Consider the following intersecting lines equations:

$$
\left\{\begin{aligned}
-m^{0} x+y & =c^{0} \\
-m^{1} x+y & =c^{1} \\
& \vdots \\
-m^{K} x+y & =c^{K}
\end{aligned}\right.
$$

As all these lines intersect in a point $\left[\begin{array}{ll}x_{i n t} & y_{i n t}\end{array}\right]$, then this system is overdetermined which unique solution is the intersection point. Developing all the system, the following infinite solutions of the intersection point can be written:

$$
\begin{array}{r}
x_{i n t}=\frac{c^{1}-c^{0}}{m^{0}-m^{1}}=\frac{c^{2}-c^{0}}{m^{0}-m^{2}}=\ldots=\frac{c^{K}-c^{0}}{m^{0}-m^{K}} \\
y_{i n t}=m^{0} x_{i n t}+c^{0}=m^{1} x_{i n t}+c^{1}=\ldots=m^{K} x_{i n t}+c^{K} .
\end{array}
$$

In an analog way, given two points the slope and intercept of the line that join them can be calculated by: 

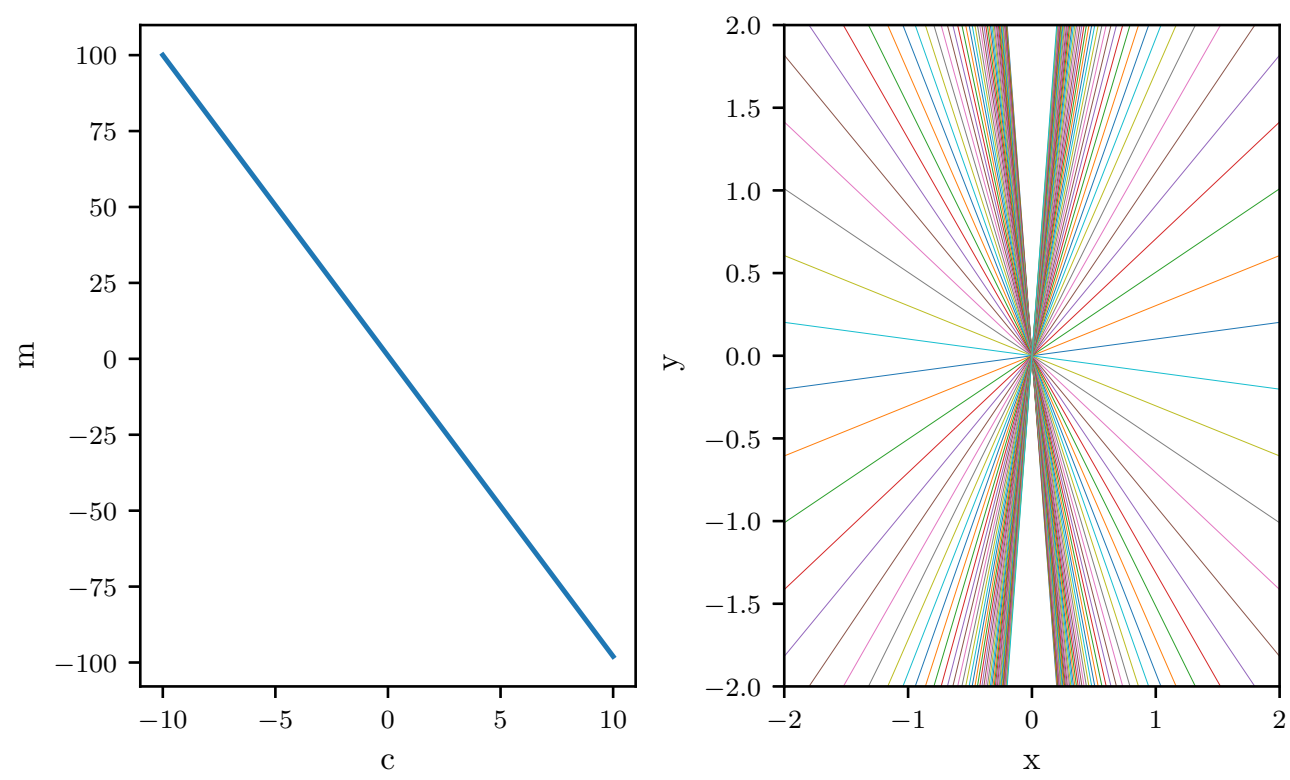

Figure 4.12: Left: Line in the $[m c]$ space. Right: $\mathrm{K}=100$ lines with slope and intercept obtained from points of the line plotted in the $[\mathrm{m}$ c] space on the left figure.

$$
\left\{\begin{array} { l } 
{ y _ { 0 } = m x _ { 0 } c } \\
{ y _ { 1 } = m x _ { 1 } c }
\end{array} \rightarrow \left\{\begin{array}{l}
m=\frac{y_{1}-y_{0}}{x_{0}-x_{1}} \\
c=x_{0} m-y_{0}=x_{1} m-y_{1}
\end{array} .\right.\right.
$$

Rewriting Eq. 4.30 the new line equations can be derived:

$$
\left\{\begin{aligned}
c_{0} & =-m^{0} x_{i n t}+y_{i n t} \\
c_{1} & =-m^{1} x_{i n t}+y_{i n t} \\
& \vdots \\
c_{K} & =-m^{K} x_{i n t}+y_{i n t}
\end{aligned}\right.
$$

All the equations in Eq. 4.32 represent the same line with slope $-x_{i n t}$ and intercept $y_{\text {int }}$. Then, it can be concluded that all the points $\left[\begin{array}{ll}m^{k} & c^{k}\end{array}\right]$ correspond, in the ideal case, to the same line.

Therefore, the objective now is to estimate this line as, the slope $-x_{\text {int }}$ and intercept $y_{\text {int }}$ of this estimated line will correspond to the 
final estimation of the intersection point. This is graphically proven in Fig. 4.12 .

Going back to the values of $\left[\begin{array}{ll}m^{k} & c^{k}\end{array}\right]$ obtained from the measurements of the IMU in each time step, an outlier detection algorithm needs to be applied to these slopes and intercepts. In order to perform an outlier detection, all the obtained pairs of $\left[\mathrm{m}^{t}, c^{t}\right]$ are clustered using the Density-based spatial clustering of applications with noise (DBSCAN) algorithm [49]. The density-based notion of clusters of this algorithm makes it ideal for the purpose of the clustering as the data is spread in very dense but disperse areas. In order to show the benefits of this clustering step, the slope and intercept of $K=500$ lines that intersect in a point are created. Then, white noise is added to these values, and the DBSCAN algorithm was applied obtaining the plot that can be observed in Fig. 4.13. Here it can be observed that this algorithm not only allows us to gather the different values of $\left[\mathrm{m}^{t}, c^{t}\right]$ in clusters, but also to discard all the values that were not assigned to any cluster and that can be considered as outliers (in pink in Fig. 4.13).

The last step in the estimation strategy is to fit a line with the points that were assigned to a cluster. However, in Fig. 4.13 is shown, in the bottom right corner of the bottom image, that there are still some outliers that were assigned to a cluster. In order to get rid of this miss classified clusters, and, at the same time, fit a line using only the inliners, the Random Sample Consensus (RANSAC) algorithm is used [20]. In Fig. 4.14 different plots from the output after applying this algorithm to the clustered pairs of $\left[\begin{array}{ll}m^{k} & c^{k}\end{array}\right]$ are shown.

Now, the estimation of the point $O_{j}^{\prime}=\left[\begin{array}{ll}x_{\text {int }} & y_{\text {int }}\end{array}\right]$ has been optimized and Eq. 4.24 can be used to calculate the distance $d_{i j}$ between the pair of quadcopters $i j$. 

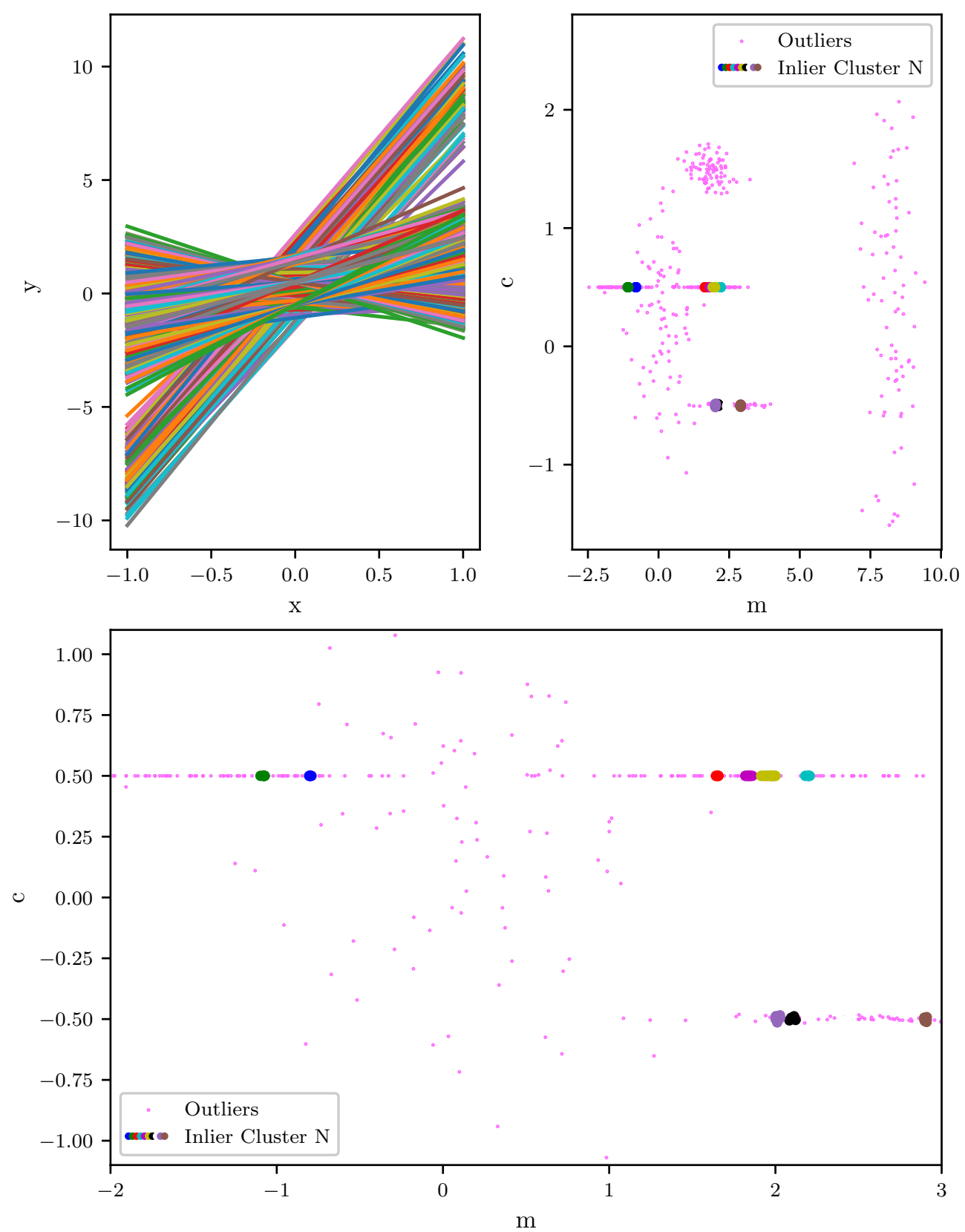

Figure 4.13: Top left: $K=500$ intersection lines with white noise added to their values of $\left[m^{k}, c^{k}\right]$. Top right: $\left[m^{k}, c^{k}\right]$ plotting in the $[m, c]$ space after applying the DBSCAN algorithm. In pink, the points of $\left[m^{k}, c^{k}\right]$ that were not assigned to any cluster. The rest of different colors correspond to cloud of points assigned to the same cluster. Bottom: Zoom in of the top right image 

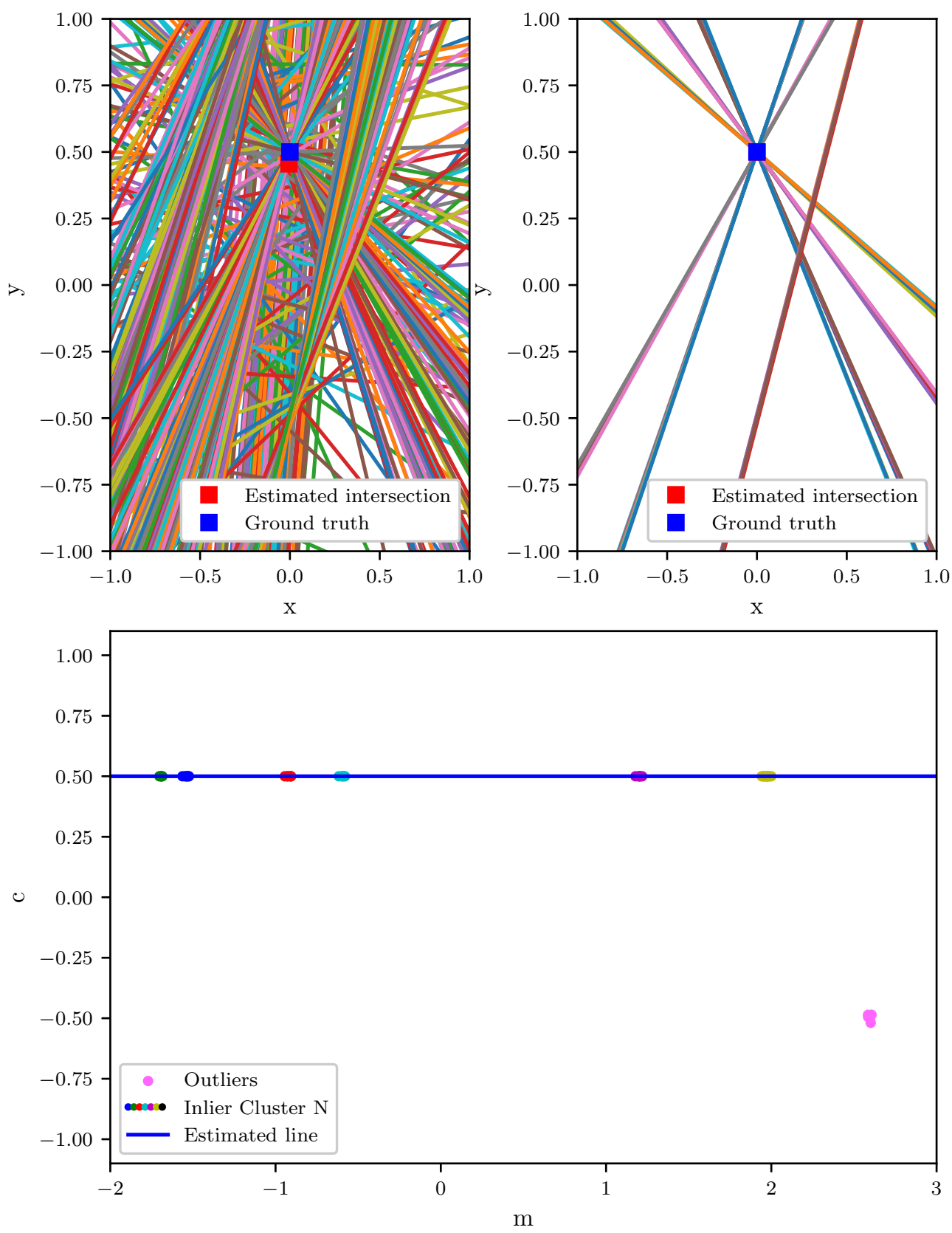

Figure 4.14: Top left: $K=500$ intersection lines with white noise added to their values of $\left[m^{k}, c^{k}\right]$. Top right: $\left[m^{k}, c^{k}\right]$ plotting in the $[m, c]$ space after applying the DBSCAN algorithm. In pink, the points of $\left[m^{k}, c^{k}\right]$ that were not assigned to any cluster. The rest of different colors correspond to cloud of points assigned to the same cluster. Bottom: Zoom in of the top right image 


\subsubsection{Absolute z-axis Rotation Within Each Pair}

Before the final positions $\mathrm{P}_{i}$ and absolute rotations $\vartheta_{i}$ are estimated, the absolute rotations $\vartheta_{i}(i)$ and $\vartheta_{i}(j)$ around the z-axis within each pair of quadcopters $i j$ need to be calculated.

Lemma 4.3.4. Let the quadcopter $i$ be chosen as the reference quadcopter within the pair ij. Defining the following vector:

$$
\mathbf{v}_{\text {norm }}=\left[\begin{array}{ll}
-1 & 0
\end{array}\right]
$$

with:

$$
\mathbf{v}_{\text {int }}=\overrightarrow{O P}_{\text {int }}=\left[\begin{array}{ll}
x_{i n t} & y_{i n t}
\end{array}\right]
$$

then the rotation around of $z$-axis of quadcopter $i$ with quadcopter $i$ as a reference is defined by:

$$
\vartheta_{i}(i)=\left\{\begin{array}{ll}
2 \pi-\operatorname{acos}\left(\mathbf{v}_{\text {norm }} \cdot \frac{\mathbf{v}_{\text {int }}}{\left\|\mathbf{v}_{\text {int }}\right\|}\right) & \text { if } \mathbf{v}_{\mathbf{y}_{\text {int }}} \geq 0 \\
\operatorname{acos}\left(\mathbf{v}_{\text {norm }} \cdot \frac{\mathbf{v}_{\text {int }}}{\left\|\mathbf{v}_{\text {int }}\right\|}\right) & \text { if } \mathbf{v}_{\mathbf{y}_{\text {int }}}<0
\end{array},\right.
$$

and the rotation around of $z$-axis of quadcopter $j$ with quadcopter $i$ as a reference is:

$$
\vartheta_{i j}=\vartheta_{j}(i)-\vartheta_{i}(i) \rightarrow \vartheta_{j}(i)=\vartheta_{i j}-\vartheta_{i}(i)
$$

Proof. Considering the vi an arbitrary convection is chosen, determining that quadcopter $i$ should be situated in the origin of this coordinate system and quadcopter $j$ along the negative x-axis, see Fig. 4.15. Quadcopter $i$ is now considered to be the reference quadcopter in the pair $i j$.

Assume the intersection point $P_{\text {int }}$ defined in Eq. 4.16 as the position of quadcopter $j$ in the virtual coordinate frame. The rotation around the z-axis of quadcopter $i$ with quadcopter $i$ as the reference quadcopter $\vartheta_{i}(i)$ is defined as the angle that $P_{\text {int }}$ must be rotated around the origin to be situated along the negative $x$-axis to satisfy the chosen convection. In the case of Fig. 4.16, the blue dot represents $P_{\text {int }}$, and it 


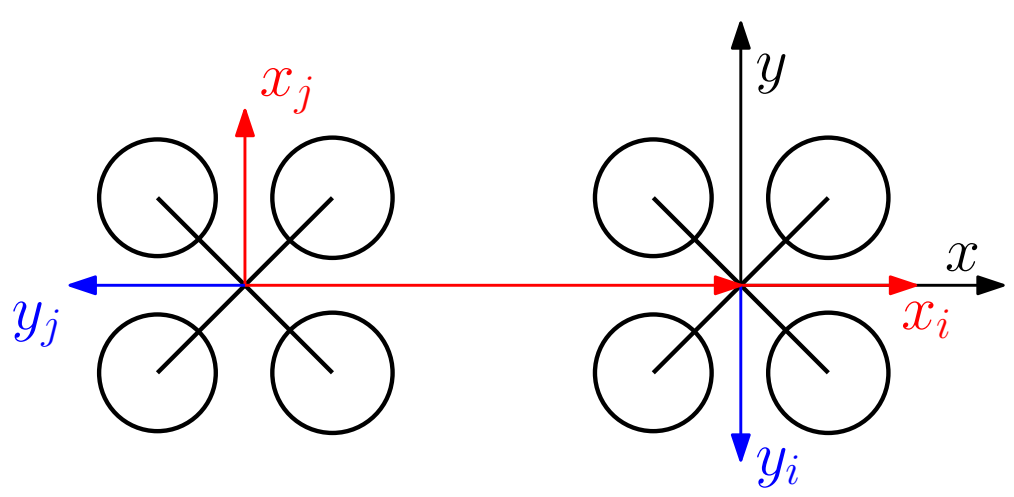

Figure 4.15: Sketch of the virtual position of a pair of Crazyflies $i j$.

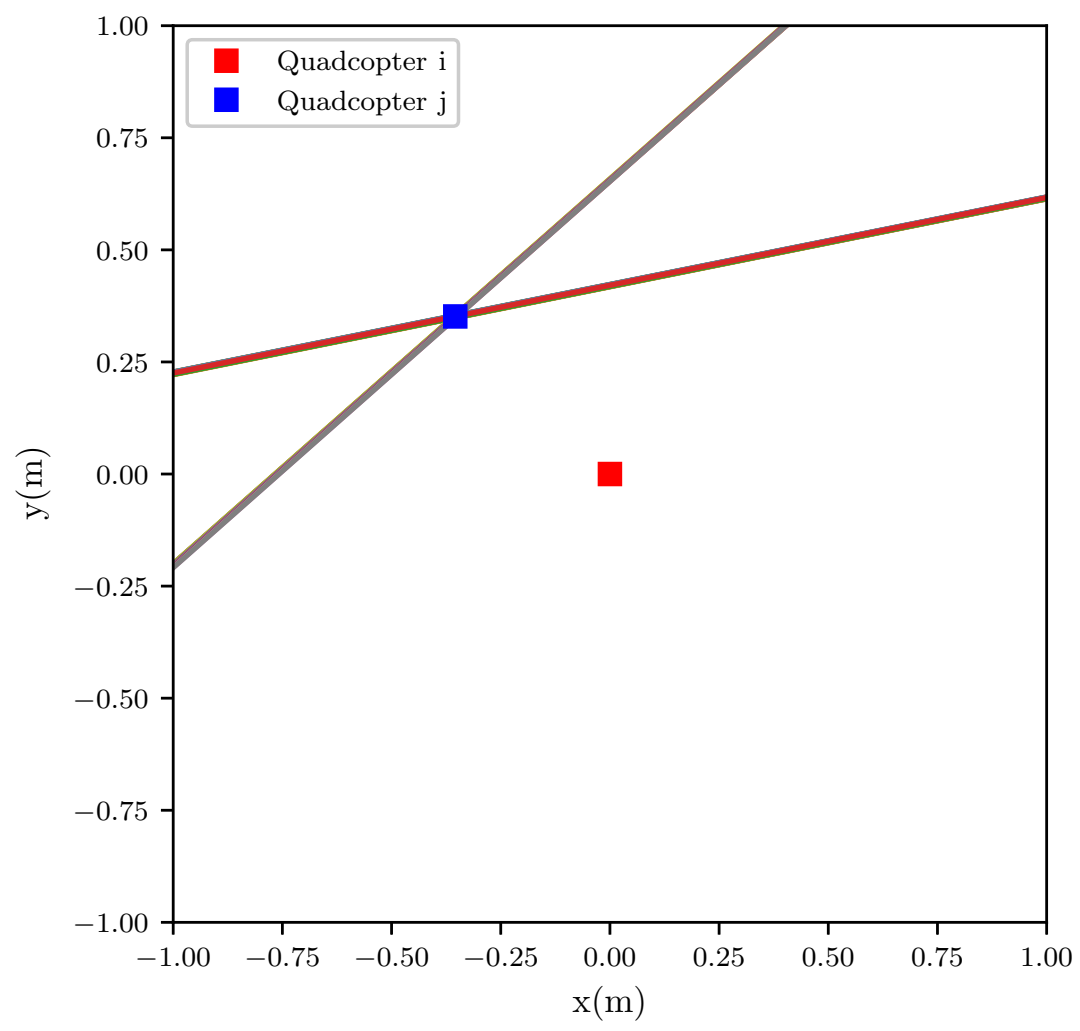

Figure 4.16: Output from the parameters estimation for the case in which $d_{i j}=0.5 \mathrm{~m}, \vartheta_{i}=45^{\circ}$ and $\vartheta_{j}=0^{\circ}$. 
has to be rotated $45 \mathrm{deg}$ in order to fulfill the requisites of the chosen convection.

Let a normal vector situated along the negative $\mathrm{x}$-axis be defined as in Eq. 4.33. The angle between to vectors $\mathbf{u}$ and $\mathbf{w}$ is expressed as:

$$
\cos \alpha=\frac{\mathbf{u} \cdot \mathbf{w}}{\|\mathbf{u}\| \cdot\|\mathbf{w}\|}
$$

Considering the vector expressed in Eq. 4.34, the angle between $\mathbf{v}_{\text {int }}$ and $\mathbf{v}_{\text {norm }}$ can be written as:

$$
\vartheta_{i}(i)=\left\{\begin{array}{ll}
2 \pi-\operatorname{acos}\left(\mathbf{v}_{\text {norm }} \cdot \frac{\mathbf{v}_{\text {int }}}{\left\|\mathbf{v}_{\text {int }}\right\|}\right) & \text { if } \mathbf{v}_{\mathbf{y}_{\text {int }}} \geq 0 \\
\operatorname{acos}\left(\mathbf{v}_{\text {norm }} \cdot \frac{\mathbf{v}_{\text {int }}}{\left\|\mathbf{v}_{\text {int }}\right\|}\right) & \text { if } \mathbf{v}_{\mathbf{y}_{\text {int }}}<0
\end{array},\right.
$$

In Lemma 4.3.1, the calculations to estimate the relative rotations $\vartheta_{i j}$ between each pair of quadcopters $i j$ are written. With these relative rotations, the rotation around the z-axis of quadcopter $j$ with quadcopter $i$ as the reference quadcopter can be directly obtained:

$$
\vartheta_{i j}=\vartheta_{j}(i)-\vartheta_{i}(i) \rightarrow \vartheta_{j}(i)=\vartheta_{i j}-\vartheta_{i}(i)
$$

Observe that the reference quadcopter can be changed from quadcopter $i$ to quadcopter $j$ using the following relations:

$$
\begin{aligned}
& \vartheta_{i}(j)=\vartheta_{i}(i)+\frac{\pi}{2} \\
& \vartheta_{j}(j)=\vartheta_{j}(i)+\frac{\pi}{2}
\end{aligned}
$$

\subsubsection{Absolute Relative Rotations and Positions}

With all the parameters that have already been estimated the system is completely defined. The last step is to gather all the obtained data to estimate the individual absolute positions $P_{i}$ and rotations $\vartheta_{i}$. The first step is to define an arbitrary coordinate frame for the system of quadcopters $S$. For simplification, this coordinate frame is chosen to 
be coincident with the coordinate frame of one of the quadcopters, the leader $L$. Using this arbitrary coordinate frame, each pair of quadcopters can be virtually rotated so that all the estimated parameters are satisfied, using Alg. 4. One all the quadcopters have been situated, the last step is to translate the arbitrary coordinate frame to the center of mass of the system $S$.

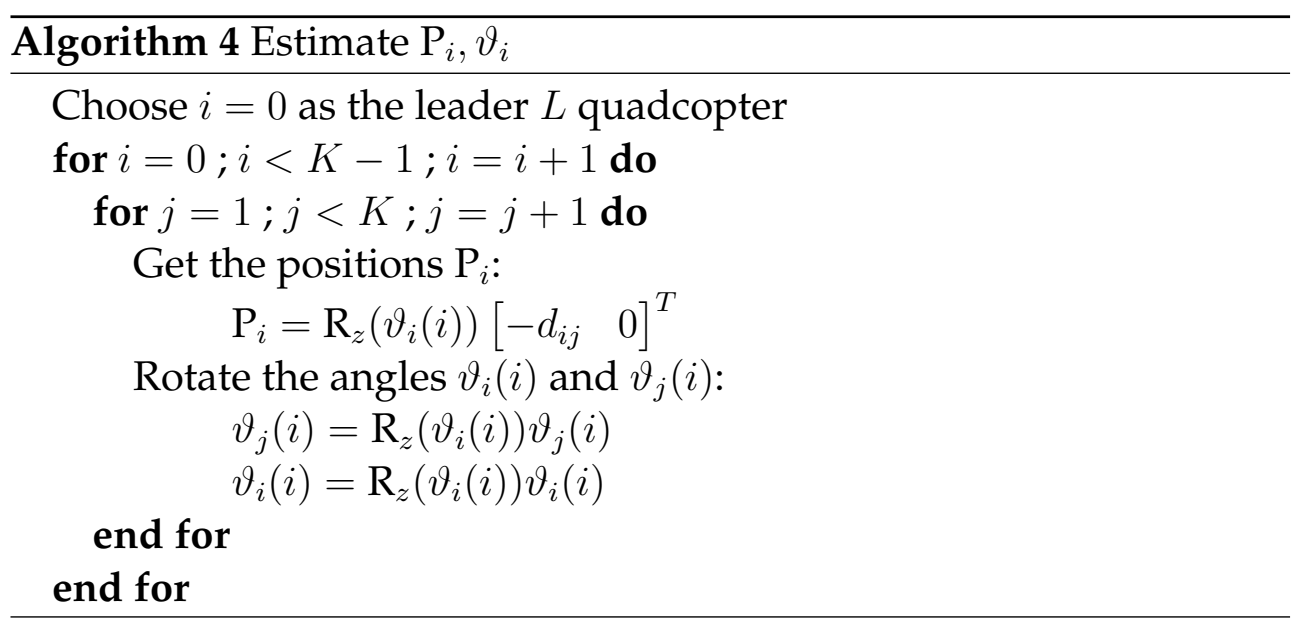

\subsubsection{Final Overview}

The fianl proposed physical parameter estimation method is shown in Fig 4.17.

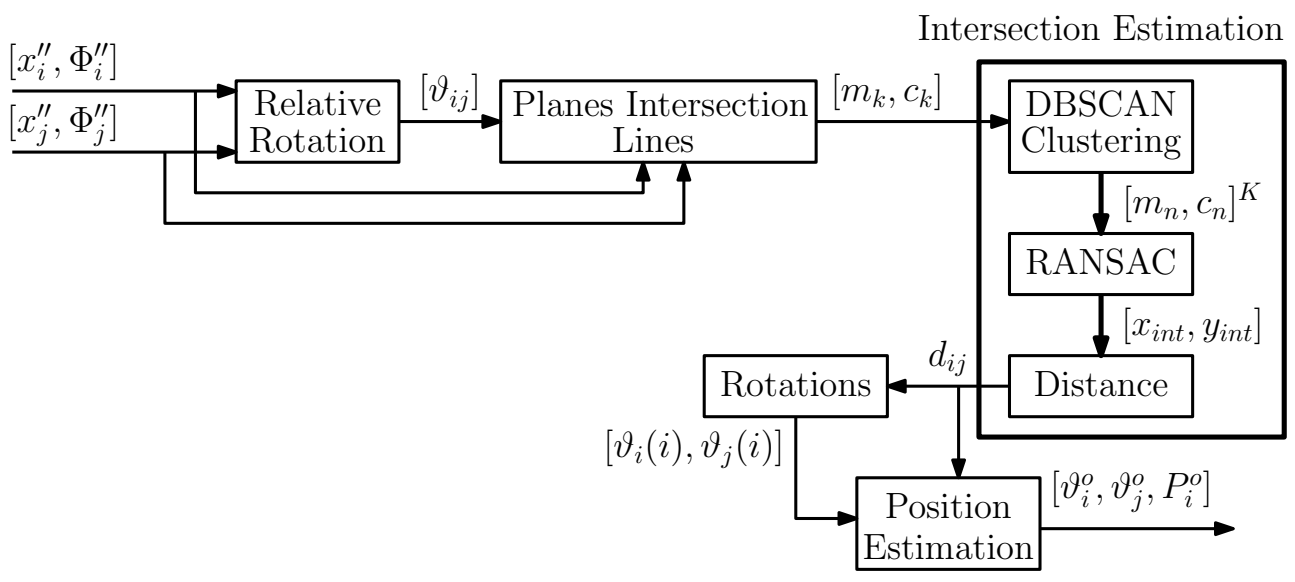

Figure 4.17: Data acquisition and preprocessing schematic approach. 


\subsection{Localization Approach}

Cooperative quadcopters use in general a localization system in which the position of all the vehicles is measured or estimated in every timestep. In most of these cooperative applications, the quadcopters are not attached with each other and each of them can move freely. This fact simplifies the localization system as each of the quads will have its own state estimation and its stability will not be affected by the estimations of the others.

On the contrary, in the case of the systems in which the quadcopters are attached, rigidly or not, the errors in the estimation of the position of each quadcopters can produce a decrement of the system stability. This situation can lead to critical stability cases if the drift in the individual errors increase in time. For instance, from a GPS module, the maximum precision that can be obtained is within the centimeter range in the best of the cases [25]. This error is too high to allow a stable flight of the system of attached quadcopters if each of them have their own GPS module.

This issue has been solved for indoor systems as the indoor position measurement systems' precision is usually high enough to find solutions that compensate for unmodeled errors in each of the quadcopters' estimation. For instance, in [41] they understand this issue as an optimization problem derived from the constraints inferred from the rigid structure that connects them. This solution on the contrary cannot by applied in the case of outdoor localization as the available sensors and modules does not provide with high precision measurements of the position. To solve this problem, the solution proposed considers that only one the quadcopters in the system will measure or estimate its position. Then, from the information obtained from the parameter estimation step, the individual positions $\mathrm{P}_{i}$ and rotations $\vartheta_{i}$ of each quadcopter, it will estimate the position of the system $S$ in real-time. This estimation is done using:

$$
\left[\begin{array}{c}
x_{S} \\
y_{S} \\
z_{S}
\end{array}\right]=\mathbf{R}_{z}\left(-\vartheta_{L}\right) \cdot\left[\begin{array}{c}
x_{L} \\
y_{L} \\
z_{L}
\end{array}\right]-\mathbf{R}_{x}\left(-\phi_{L}\right) \mathbf{R}_{y}\left(-\theta_{L}\right) \mathbf{R}_{z}\left(-\psi_{L}\right) \cdot\left[\begin{array}{c}
\mathbf{P}_{x_{L}} \\
\mathbf{P}_{y_{L}} \\
\mathbf{P}_{z_{L}}
\end{array}\right]+\left[\begin{array}{c}
\mathbf{P}_{x_{L}} \\
\mathbf{P}_{y_{L}} \\
\mathbf{P}_{z_{L}}
\end{array}\right]
$$

with, $\left[\begin{array}{lll}x_{S} & y_{S} & z_{S}\end{array}\right]^{T}$ the estimated current position of the system $S$, $\left[\begin{array}{lll}x_{L} & y_{L} & z_{L}\end{array}\right]^{T}$ the estimated current position of quadcopter $L, \vartheta_{L}$ the 


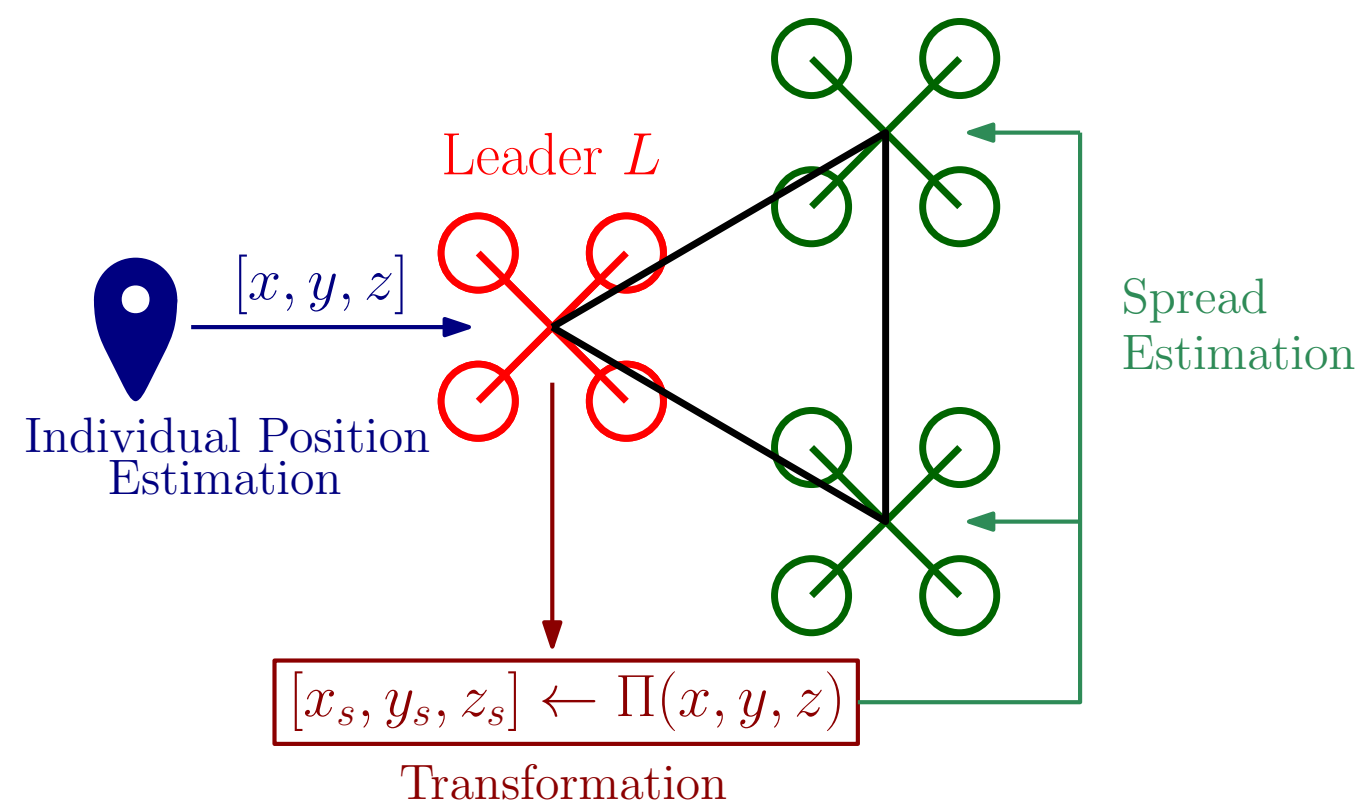

Figure 4.18: Schematic representation of the proposed localization approach.

estimated absolute rotation around the z-axis of the leader quadcopter $L$ relative to the system $S,\left[\begin{array}{lll}\phi_{L} & \theta_{L} & \psi_{L}\end{array}\right]^{T}$ the estimated current attitude of the leader quadcopter $L,\left[\begin{array}{lll}P_{x_{L}} & P_{y_{L}} & P_{z_{L}}\end{array}\right]^{T}$, the position of the quadcopter $L$ relative to the system $S$ coordinate frame.

Finally, this position will be spread to the rest of quadcopters attached to the system $S$. Even though this method generates a delay in the system due to the inevitable latency in the information sending process, it will be shown in the experimentation that it does not affect the final performance of the system. Fig. 4.18 represents the simplified proposed solution.

The estimation of the rest of parameters of the full-state of the system $S$ is achieved individually in each quadcopter using only the measurements obtained from the IMUs. In the case of the linear velocities, they are transformed taking into account the linear and angular velocities and the position and rotation of each quadcopter. In the case of the angular velocities and attitude, the individual estimations need to be rotated according to the estimated value of $\vartheta_{i}^{o}$ for each quadcopter to be adapted to the system $S^{\prime}$ coordinate frame. The equations used to perform the transformation from the leader current linear velocities estimation $L$ to the system $S$ current linear velocities estimation are: 


$$
\begin{aligned}
{\left[\begin{array}{c}
\tilde{v}_{x_{S}} \\
\tilde{v}_{y_{S}} \\
\tilde{v}_{z_{S}}
\end{array}\right] } & =\mathrm{R}_{z}\left(-\vartheta_{L}\right) \cdot\left[\begin{array}{l}
v_{x_{L}} \\
v_{y_{L}} \\
v_{z_{L}}
\end{array}\right] \\
\Gamma & =\arccos \frac{\mathrm{P}_{y_{L}}}{d_{C G}}+\psi_{L} \\
v_{x_{S}} & =\tilde{v}_{x_{S}}-w_{z_{L}}\left\|\left[\begin{array}{lll}
\mathrm{P}_{x_{L}} & \mathrm{P}_{y_{L}} & \mathrm{P}_{z_{L}}
\end{array}\right]^{T}\right\| \cos (\Gamma) \\
v_{y_{S}} & =\tilde{v}_{y_{S}}-w_{z_{L}}\left\|\left[\begin{array}{lll}
\mathrm{P}_{x_{L}} & \mathrm{P}_{y_{L}} & \mathrm{P}_{z_{L}}
\end{array}\right]^{T}\right\| \sin (\Gamma) \\
v_{z_{S}} & =\tilde{v}_{z_{S}}+\left|\mathrm{P}_{y_{L}}\right| \phi_{L}-\left|\mathrm{P}_{x_{L}}\right| \theta_{L}
\end{aligned}
$$

with $\left[\begin{array}{lll}v_{x_{L}} & v_{y_{L}} & v_{z_{L}}\end{array}\right]$ the estimated linear velocities of the system $L$ and $w_{z_{L}}$ the current angular velocity around the z-axis of quadcopter $L$. As the connection is rigid, to transform the angular velocities and attitude measured in quadcopter $L$ into the estimation of these parameters of the system $S$, they only need to be rotated according to the estimated parameter $\vartheta_{L}$ of quadcopter $L$ :

$$
\begin{gathered}
{\left[\begin{array}{c}
\phi_{S} \\
\theta_{S} \\
\psi_{S}
\end{array}\right]=\mathrm{R}_{z}\left(-\vartheta_{L}\right) \cdot\left[\begin{array}{l}
\phi_{L} \\
\theta_{L} \\
\psi_{L}
\end{array}\right]} \\
{\left[\begin{array}{l}
w_{x_{S}} \\
w_{y_{S}} \\
w_{z_{S}}
\end{array}\right]=\mathrm{R}_{z}\left(-\vartheta_{L}\right) \cdot\left[\begin{array}{l}
w_{x_{L}} \\
w_{y_{L}} \\
w_{z_{L}}
\end{array}\right]}
\end{gathered}
$$




\section{Chapter 5}

\section{Controller Design for Rigidly At- tached Quadcopters}

Using all this information gathered in the previous section, it is possible to design a general control strategy that takes this information as an input in order to adapt to different configurations. This allows the system $S$ to achieve a stable and efficient flight independently of the individual position $\mathbf{P}_{i}^{o}$ and rotation around the $z$-axis $\vartheta_{i}^{o}$ of each quadcopter $i$ and the number of quadcopters $N$.

\subsection{Control Architecture and Strategy}

In this section, the implementation of the control strategy and architecture is described in detail.

\subsubsection{Control Architecture}

The first step is to design the general controller architecture. This architecture will be based on the already implemented in the Crazyflie quadcopter [7] that will be used for the experimentation, see Chapter 6. The position $\left[x_{s}, y_{s}, z_{s}\right]$ will be controlled using 4 groups of PIDs in cascade (see Section 2.3). The inner loop corresponds to the angular velocities $\mathrm{w}_{s}$ control loop, followed by the attitude $\phi_{s}$, the linear velocity $\mathbf{v}$ and position $\mathbf{p}$ control loops. The position $\mathbf{p}$ is controlled using a proportional controller, the linear velocities $\mathbf{v}$, with a proportionalintegral controller, and the attitude $\phi_{s}$ and angular velocities $\mathrm{w}_{s}$ with proportional-integral-derivative controllers. However, these control 
loops will be gathered in to main groups: the attitude $\phi_{s}$ and angular velocities $\mathbf{w}_{s}$ control loops that will run at the same frequency (500 $\mathrm{Hz})$ and the position and linear velocities control loops $(100 \mathrm{~Hz})$. This is justified by the fact that the changes in the attitude and angular velocities are much more reactive than changes in the position or linear velocities. The final structure is represented in Fig. 5.1. This way, there is a controller for each of the parameters in the full-state of the system $S$ :

$$
\left[x_{s}^{t}, y_{s}^{t}, z_{s}^{t}, \dot{x}_{s}^{t}, \dot{y}_{s}^{t}, \dot{z}_{s}^{t}, \phi_{s}^{t}, \theta_{s}^{t}, \psi_{s}^{t}, w_{x_{s}}^{t} w_{y_{s}}^{t}, w_{z_{s}}^{t}\right]^{\top},
$$

where the controller is composed of 12 PIDs which gains need to be tuned for each combination of initial positions $\mathbf{P}_{i}^{o}$ and rotations around the $z$-axis $\vartheta_{i}^{o}$ of each quadcopter $i$ and the number of quadcopters $N$. Each of the controllers, position, linear velocities, attitude and angular velocities, needs to obtained the desired behavior of these parameters in each of the 3 axis $x, y$ and $z$. As a consequences, each of these controllers needs a PID controller for each of the mentioned axis, with a total of 12 PIDs to tune.

Fig. 5.1 shows that the main inputs for the controller are the position $[x, y, z]$ and yaw $\psi$ set-points. The $z$ position and $v_{z}$ linear velocity controllers form the so called height controller that estimates the needed thrust $z_{\text {thrust }}$ that the system needs in order to reach and maintain the desired height. Besides, between the angular velocities controllers' blocks and the system's PWM input there is a block called Power distribution. This block maps the values $[R, P, Y]$ calculated by the angular velocities controllers and the needed thrust $z_{\text {thrust }}$ with the $\mathrm{PWM}_{i j}$ signals that need to be applied to each propeller $i j$. The vector $[R, P, Y]$ can be understood as the proportional value of the moments $\left[M_{x}, M_{y}, M_{z}\right]$ that need to be applied around each axis in order to obtained the desired attitude.

$$
[R, P, Y]=k\left[M_{x}, M_{y}, M_{z}\right]
$$

This block is the key that allows this control structure to be scalable to every possible combination of $\vartheta_{i}^{o}$ by just changing its inner parameters. It is necessary to transform this block so it is able to map the vector $\left[R, P, Y, z_{\text {thrust }}\right]$ to the $\mathrm{PWM}_{i j}$ values for each propeller $i j$ in the system of $N$ quadcopters. 


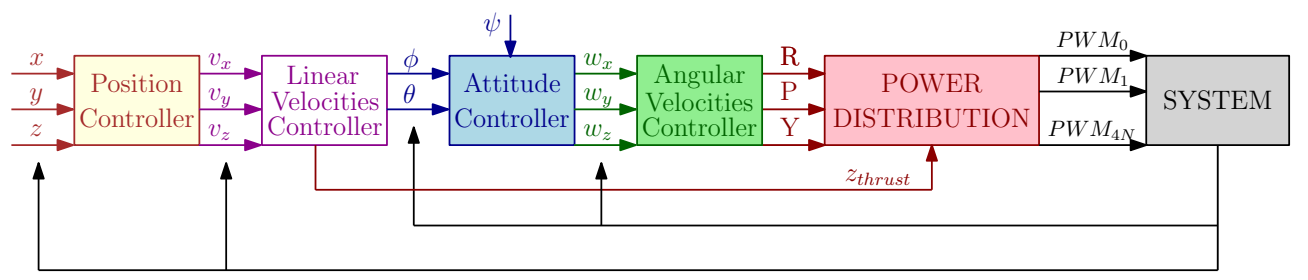

(a)

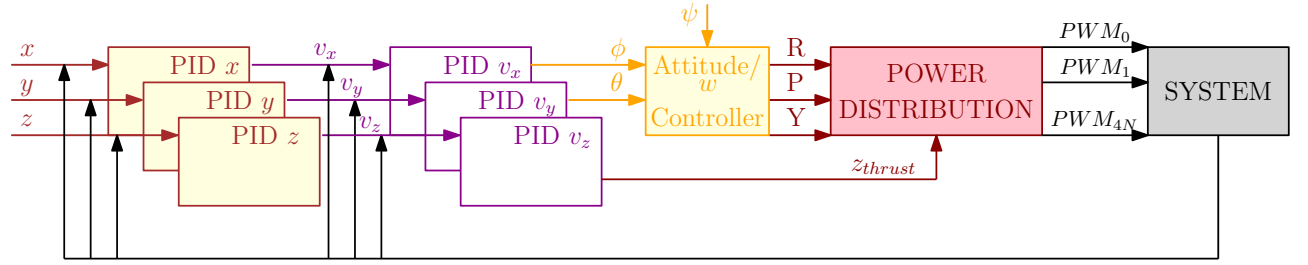

(b)

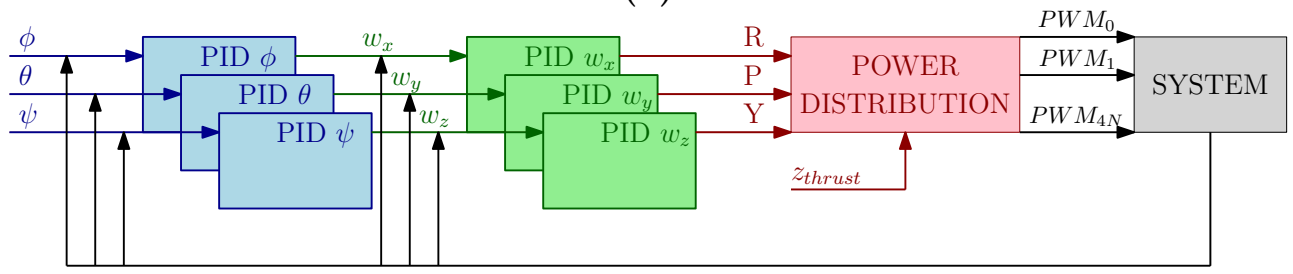

(c)

Figure 5.1: (a) Generalized control law structure for the multiquadcopter system. (b) Outer Group: Position and linear velocities control loops. (c) Inner Group: Attitude and angular velocities control loops.

Once the general loop structure has been defined, the gains of the PID controllers have to be tuned. Then, each quadcopter will have the same exact PID structure on-board as the one in Fig. 5.1, but the PID gains will change in each quadcopter depending only on its position $\mathbf{P}_{i}^{o}$. Then, each roll $\phi$, pitch $\theta$, yaw $\psi$ angular velocity around the $x$-axis $w_{x}, y$-axis $w_{y}$ and $z$-axis $w_{z}$ PID controllers will get different value of their $k_{p}, k_{i}$ and $k_{d}$ gains depending on the distances the quadcopter $i$ to each axis. It can be considered as an adaptive controller structure, in which the gains of the controllers will depend on the parameters that are calculated using the method proposed in Section 4 .

\subsubsection{Controller Design}

However, it is not efficient to calculate the gains for every possible configuration. As these parameters will depend solely on the distance to 


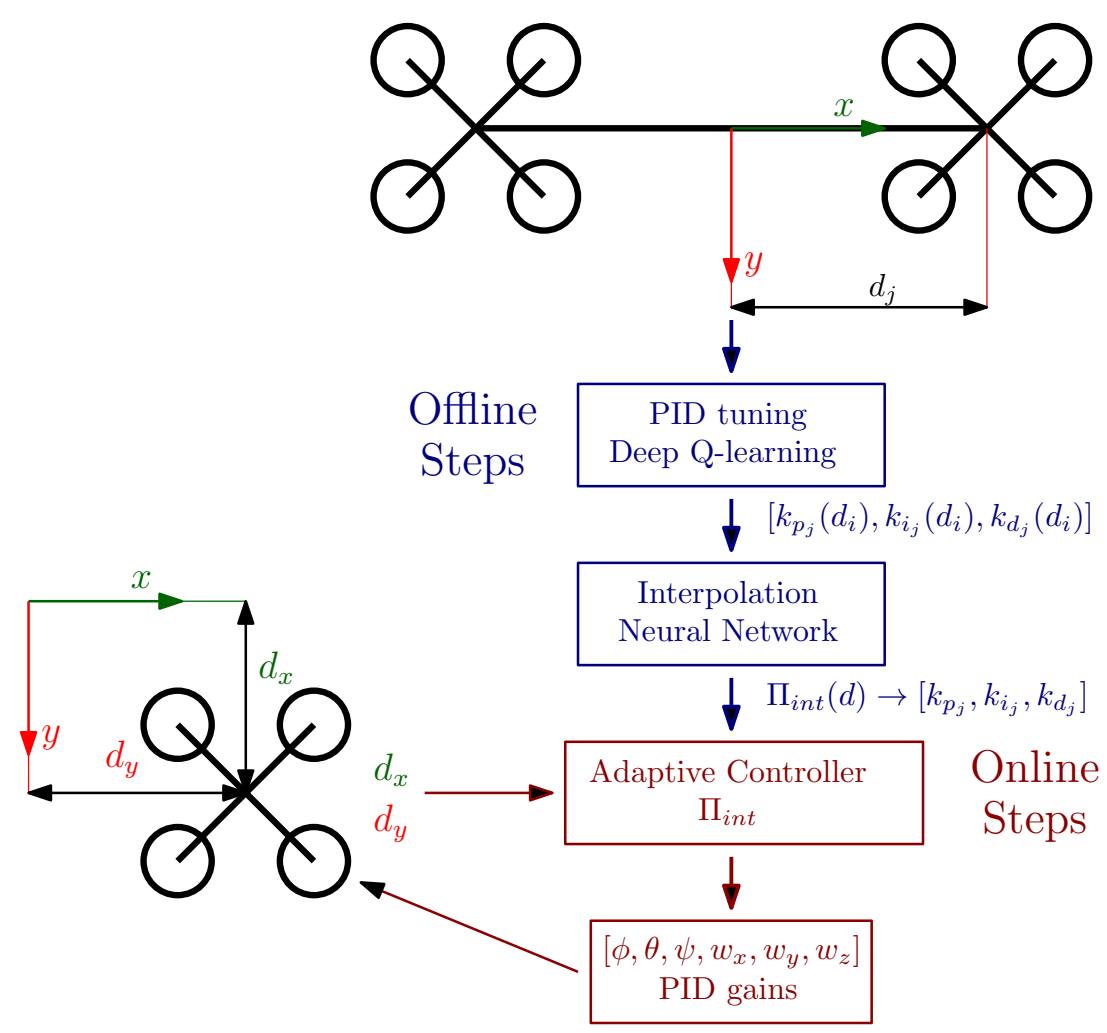

Figure 5.2: Schematic control strategy

each axis, the system can be simplified to a 2 quadcopters system in which only the distance to one of the $x$ or $y$-axis is not zero. The idea is to tune the PID gains in simulation for this 2 quadcopter system for some distances chosen from a wide range and using some interpolation method to estimate the gains for the distances that were not used in the PID tuning step. Then, in the real system $S$, after the distances to each $x$ and $y$-axis $d_{x}$ and $d_{y}$ have been estimated using the method of Section 4, they can be used to obtained the interpolated PID gains for the $\phi, \theta, \psi, w_{x}, w_{y}$ and $w_{z}$ PID controllers gains (see Fig. 5.2).

Only the PIDs that controls $\phi, \psi, w_{x}$ and $w_{z}$ will be tuned as the quadcopters are considered to be initially situated along the $y$-axis.

\subsection{Controller Design}

The first step is to modify the Power Distribution block so the controller is able to adapt the different individual rotations around the z-axis $\vartheta_{i}$ 


\section{QUADCOPTERS}

of each quadcopter.

The generated momentum in the $x, y$-axis will be dependent on the distance of the propeller to each axis $\left[d_{x_{i j}}, d_{y_{i j}}\right]$ and its position $\mathbf{p}_{i j}=$ $\left[x_{i j}, y_{i j}\right]$. Both parameters can be obtained from Eq. 3.8 and having that:

$$
\left[\begin{array}{l}
d_{x_{i j}} \\
d_{y_{i j}}
\end{array}\right]=\left[\begin{array}{l}
\left|y_{i j}\right| \\
\left|x_{i j}\right|
\end{array}\right]
$$

and the general expression of the momentum:

$$
m=F d,
$$

then, the further a propeller is from one axis, the bigger the momentum generated around that axis for the same value of PWM. To include this phenomena in the Power distribution block, the normalized distances of each propeller to each axis will be included in the equations:

$$
\left[\begin{array}{l}
d_{x_{i j}}^{n o r m} \\
d_{y_{i j}}^{n o r m}
\end{array}\right]=\left[\begin{array}{c}
\frac{d_{x_{i j}}}{\max _{x}} \\
\frac{d_{y_{i j}}}{\max \mathbf{d}_{y}}
\end{array}\right] .
$$

It is also necessary to calculate the sign of the momentum generated by each propeller $i j$. In the case of the momentum generated by a propeller around the $z$-axis, it is directly produced by the momentum of each spinning propeller (see Eq. 3.7). Therefore, the momentum around the $z$-axis generated by each propeller will not depend on its distance to this axis, but the spinning direction of the propeller. The sign of this direction for each propeller $i j$ will be expressed by $s_{i j}$. Then, the equations inside the final Power distribution block are expressed by:

$$
\mathrm{PWM}_{i j}=z_{\text {thrust }}+\frac{y_{i j}}{\left|y_{i j}\right|} d_{x_{i j}}^{\text {norm }} r+\frac{x_{i j}}{\left|x_{i j}\right|} d_{y_{i j}}^{n o r m} p+s_{i j} y .
$$

Using this approach, the final controller PID gains will not be affected by the relative rotation of each quadcopter around its $z$-axis $\vartheta_{i}^{o}$ but only by its distances to the $x$ and $y$-axis. This will be the parameters that will considerably change the inertia of the final system $S$ and the maximum moments that can be obtained. 
The different gains of the PID controllers need to be tuned for each different configurations as the inertia and the maximum and minimum momentum around the main axis by each propeller will vary. To solve this problem, two solutions were proposed:

- Online Automatic Tuning: once the main parameters $\mathrm{P}_{i}$ and $\vartheta_{i}$ have been estimated, the idea would be to tune the PID gains right after. For this, several methods could be used: gaussian processes [4], Reinforcement Learning [15] techniques or even classic methods such as Ziegler and Nichols [79]. However, most of these methods are still slow, and hard to implement.

- Offline Automatic Tuning: in this case, the PID tuning is done offline, for different general configurations. Then, the obtained gains for these different configurations are interpolated. This interpolation function can be use to directly extract the values of the gains for any other possible configuration. The main advantage with this method is that the gains are obtained inmediatly after the parameters $\mathrm{P}_{i}$ and $\vartheta_{i}$ have been estimated, just using the interpolation function. However, that obtained gains would not be as optimal as in the first case, and the number of configurations that need to be tuned offline, and their design well designed in order to ensure a correct interpolation step.

In the current thesis, only the second case will be implemented, leaving the study of the first case as a future work.

\subsection{Controller Tuning}

There are different PID-tuning techniques with a variety of withdraws and advantages. However, most of them are based on manual methods. In this case, it is required an automatic tuning method that is able to satisfy certain behavior requirements: limited maximum overshooting and short peak time. As it will be mentioned in Section 8, one future improvement for the presented approach is to add an online Reinforcement Learning method (see Section 2.5) in order to fine tune the already estimated PID gains while the system is flying. This would be important as it will improve the flight performance and suppress any un-optimal behavior derived from the possible errors in the initial estimation of $\mathbf{P}_{i}^{o}$. 
In order to try a possible approach that can be used in the future implementation of this on-line reinforcement learning based optimizer, it was decided to tune the PID's gains using a reinforcement learning method. One possible approach that was considered was PILCO algorithm [15]. This method first estimates the gaussian model of the system and then, using gradient descent, the optimal PID gains for the estimated model. However, this approach has only been tested in simpler system like the inverted pendulum or a bidirectional cart, and the system proposed in the current thesis is much more complex, as more controllers need to be tuned, and the convergence to a optimal solution would be slow. The final chosen approach is based on Q-learning methods.

\subsubsection{Reinforcement Learning PID Tuning: Deep Q- Learning}

The main concepts of Reinforcement Learning, more precisely of Deep Q-learning, are explained in Section 2.5. Based on this basic concepts, the main proposed method can be developed.

In the case of the PID gains optimization problem using Deep Qlearning, several considerations need to be taken into account. The agent will be the PIDs themselves. The actions $a$ will be to increase, decrease or not change the values of the gains and the state $s$ is represented by the current gains of the PIDs. The objective is to tune the PID gains for different chosen distances $d$. There are then four agents, one for each PID, which states can be defined as:

$$
\begin{aligned}
& s_{t}^{\phi}=\left[\begin{array}{lll}
K p_{t}^{\phi} & K i_{t}^{\phi} & K d_{t}^{\phi}
\end{array}\right] \\
& s^{w_{x_{t}}}=\left[\begin{array}{ll}
K p^{w_{x_{t}}}, K i^{w_{x_{t}}}, K d^{w_{x_{t}}}
\end{array}\right] \\
& s_{t}^{\psi}=\left[K p_{t}^{\psi}, K i_{t}^{\psi}, K d_{t}^{\psi}\right] \\
& s^{w_{z_{t}}}=\left[K p^{w_{z_{t}}}, K i^{w_{z_{t}}}, K d^{w_{z_{t}}}\right]
\end{aligned}
$$

The total number of gains that need to be tuned for each PID is 3. Then, there are 9 possible actions for each agent, or PID. The gains of each agent $k$ can be expressed by $k_{k}^{t}$ for each time step $t$. Then the evolution 
of each gain can be represented as:

$$
k_{k}^{t+1} \Rightarrow\left\{\begin{array}{l}
k_{k}^{t}\left(1+\alpha_{t}\right) \\
k_{k}^{t}\left(1-\alpha_{t}\right) \\
k_{k}^{t}
\end{array},\right.
$$

where $\alpha_{t}$ represent the increment or decrement percentage of the gain in each time step. It was decided to use a decremental value of $\alpha_{t}$ so the tuning of the gains gets finer as the algorithm is reaching the convergence. Then $\alpha_{t}$ can be defined as:

$$
\alpha_{t} \Rightarrow\left\{\begin{array}{ll}
\alpha_{t-1} \delta & \text { if } t>0 \\
\alpha_{t}=0.1 & \text { if } t=0
\end{array},\right.
$$

with $\delta$ chosen experimentally. In this case the chosen $\delta$ was 0.995 .

However, instead of tuning each of the PIDs separately, and in order to accelerate the learning process and find the optimal set of parameters, the tuning of the $\phi$ and $w_{x}$ PIDs controllers' gains will not be done together but separately from the $\psi$ and $w_{z}$ ones. Therefore, the learning algorithm will be applied twice: one for the pair of $\phi$ and $w_{x}$ controllers and one for the $\psi$ and $w_{z}$ controllers. For each time step, two actions will be chosen from the policy, one to change the state of the attitude PID's parameters $(\phi$ or $\psi$ ) and one to change the angular velocities PID's parameters $\left(w_{x}\right.$ or $\left.w_{z}\right)$.

One main difference with the traditional Deep Q-learning algorithm is that, if the predicted action derives to a worse state (smaller reward and therefore, worse estimated PID), then the PID is set back to the PID that highest reward has obtained so far. This is important if the algorithm is ever implemented in a real system to be run on-line, other wise, the system could reach a worst gains configuration that can cause instability or even the crash of the system.

This approach simplifies the complexity of the problem, but can only be applied as the PIDs gains tuning will be done in simulation. If the same approach is implemented in the real system and run on-line, it will be needed to include some a priori safety constraints during the learning or even learn this constraints on-line. In [1], [61] and [24] different methods to learn this safety constrains on-line while performing the main reinforcement learning algorithm are proposed.

It is important to chose a wise distances range that will be used for the gains tuning. The distance range chosen will start at the mini- 
mum distance possible between both quadcopters and the maximum distance in which the flight is still stable, studying the mathematical limitations of the system of 2 quadcopters. Then, some evenly spread distances have to be chosen from this calculated range, and the gains will be tuned for those distances. To accelerate the learning process, once the PID gains has been tuned for the shorter distance, these gains will be used as the initial state for the next used distance. This process is iterated until the longer and last distance has been used.

One important factor that will affect the speed of the learning process is the initial values of these PID gains. The idea is to choose the PID gains of the single-quadcopter controller if it has the same structure as the one proposed in Fig. 5.1. If these is not the case, then the optimal PID gains needs to be tuned for the single-quadcopter case. As the first distance chosen is the shorter possible one, the behavior, even though it will not be optimal, is supposed to be stable and the convergence then will be reached quicker than if the initial state is chosen randomly.

\section{Neural Network Structure}

The main concepts and terminology related to Neural Networks are explained in Section 2.4. The input of the Neural Network, or $Q$ Network will be the current state $s_{t}$. As it was said, the optimization of the $\phi$ and $w_{x}$ PID gains will be done together, in the same way as in the $\psi$ and $w_{z}$ PID gains case. Then, $2 Q$-Networks will be trained per distance chosen. For each case, the state will be have a size of 6 inputs. The output of the $Q$-Network represents the expected reward of each possible action $a$. There are 3 possible actions for each gain, or input, having this way, the network, 18 outputs. The size of the Neural Network will also determine how fast the learning process will be and even, if the convergence can be reached or now. Experimentally, it was determined that an optimal structure of the Neural Network to ensure a fast convergence was composed by two hidden layers of 16 units in each of them. The hidden units are set to be Rectified Linear Units (ReLU) [55], explained in Section 2.4. The output layer, with 18 units, has linear activation units. The final structure is represented in Fig. 5.3 . 


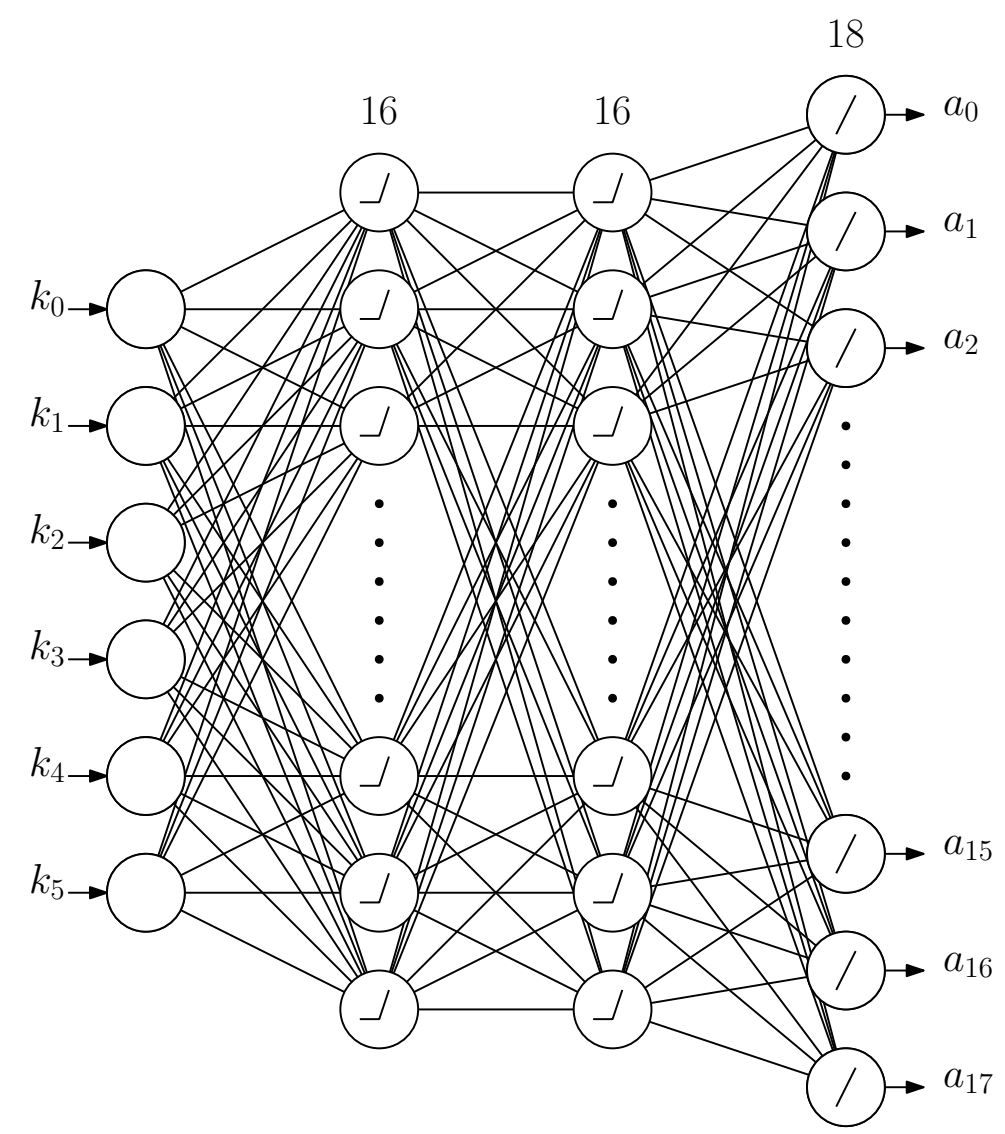

Figure 5.3: Q-Network structure.

\section{Q-Network Training}

The output layer shown in Fig. 5.3 outputs the expected reward from each of the 18 possible actions. This actions can be divided in 2 groups, the one that changes the gains for the attitude controllers for $\phi$ or $\psi$ and the ones for the angular velocities controllers for $w_{x}$ and $w_{z}$, having 9 possible actions in each group. From each group, the action with a higher expected reward will be chosen (see Eq. 2.36) and will trigger the transition from state $s_{t}$ to $s_{t+1}$. This actions are called to be on-policy actions. When the actions are chosen, on the contrary, randomly, they are considered off-policy actions.

The training of the Q-Network is achieved performing on-policy or off-policy rollouts. A rollout is set of consecutive possible states $s_{0}^{R}$ to $s_{N}^{R}$ that are obtained selecting one of the possible actions in each state $s_{i}^{R}$. The length of a rollout is determined by the number of states that 


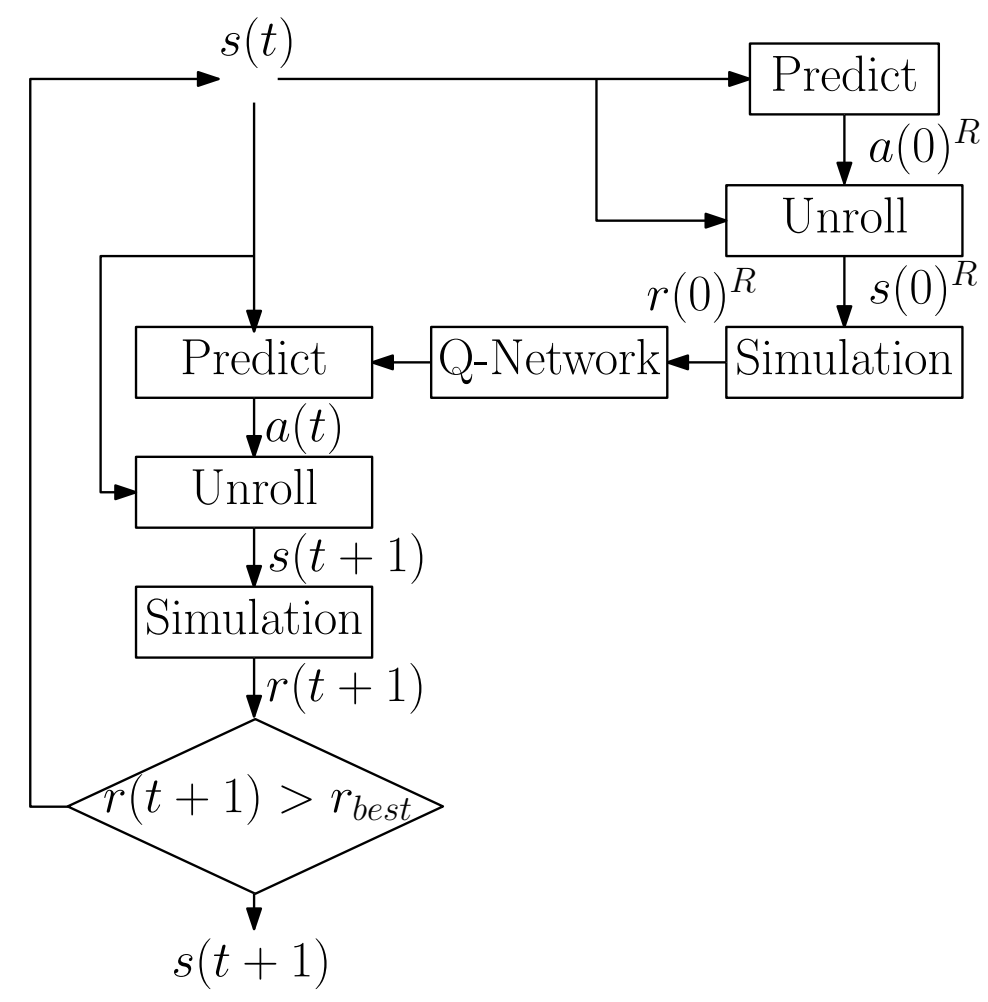

Figure 5.4: Q-Network training simplified approach.

were explored. They are consider to be on-policy rollouts if the actions were chosen using the policy $\pi\left(s_{i}^{R}\right)$ (Eq. 2.36) or off-policy otherwise.

Then, the training algorithm is simple, and it is simplified in Fig. 5.4. Given the current state $s_{t}$, perform a rollout of length $N$. With the rewards $r_{i}^{R}$ obtained by each state and chosen action pairs $\left[s_{i}^{R}, a_{i}^{R}\right]$, train the Q-Network. Once this rollout has been trained, the action $a_{t}$ is chosen from the policy:

$$
a_{t}=\operatorname{argmax}_{a} Q\left(s_{t}, a ; \theta\right),
$$

triggering the transition to the state $s_{t+1}$. This way, the state will evolve until reach the convergence.

The rollout lenght does not need to be long in this case as the set of actions and states is not complex. A 1-step length rollouts are used to ensure fast convergence. In each iteration of the learning algorithm, two main steps can be considered: prediction, in which the next actions for the rollouts are chosen (on-policy or off-policy) and training, 
in which the rewards obtained in the rollout are trained to the QNetwork.

To ensure a good exploration of the state space, the prediction step is done initially randomly. With the number of iterations, the probability of the prediction being done randomly (off-policy) decreases, following the next expression, for each iteration $i$ :

$$
a_{i}^{R} \leftarrow \begin{cases}\operatorname{argmax}_{a} Q(s, a) & \text { if } X \sim U(0,1)>=\Gamma_{k} \\ a \in A & \text { if } X \sim U(0,1)<\Gamma_{k}\end{cases}
$$

where $\Gamma_{k}$ is:

$$
\Gamma_{k}=\left\{\begin{array}{ll}
1 & \text { if } k=0 \\
\Gamma_{k-1} 0.99 & \text { if } k>0
\end{array} .\right.
$$

Then, with the chosen action, the system evolves to the next state of the rollout $s_{i+1}^{R}$. The reward of this state is calculated and stored, getting, for each 1-step length rollout the following information:

$$
\left[s_{0}^{R}, a_{0}^{R}, r_{0}^{R}\right],
$$

where the transition between the state $s_{t}$ and $s_{0}^{R}$ is triggered by the action chosen in Eq. 5.13. Then, the training of the Q-Network can be represented by the next expression:

$$
Q\left(s_{t}, a_{t}\right) \sim r_{0}^{R}+\gamma \max _{a} Q\left(s_{0}^{R}, a_{0}^{R}\right) .
$$

The last step is to predict the action $a_{t}$ that triggers the evolution to new state $s_{t+1}$ after the training of the current rollout has been done. All the process is iterated until the convergence is reached.

\section{Reward Function}

The convergence will depend on the design of the so called reward function. In this case, its design can be complex as the system must not only have a stable behavior, but also to respond within certain constraints. The objective is to decrease both the maximum overshooting and the peak time of the response of the controllers. 
To obtain enough information to estimate the reward of the current state, it is necessary to simulate the system long enough so significant data is obtained. To calculate the reward of a certain state $s$, an arbitrary set-point for the angle which PIDs' gains are wanted to be tuned is chosen. In this case, the set-point will be the maximum attitude angle possible $(25 \mathrm{deg})$ in the case of the $\left[\phi, w_{x}\right]$ controllers and a angle of $45 \mathrm{deg}$ in the case of the $\left[\psi, w_{z}\right]$ controllers. Then, the simulation is run until the oscillations around the setpoint are smaller than a $2.5 \%$ or simulation time is greater than a maximum value chosen experimentally. This value was set to $4 \mathrm{~s}$ in simulation time for the case of the yaw $\psi$ and angular velocity around the z-axis $w_{z}$ controller's tuning and $1.5 \mathrm{~s}$ otherwise. The response around the $\mathrm{z}$-axis is slower as the maximum applied momentum in that axis is much smaller than in the $x$ and $y$ axis. This values were chosen as any response over this time was to slow to ensure a correct performance of the system. For each estimated state, we will simulate the system from a initial height of $\mathrm{z}$ $=1 \mathrm{~m}$ and all the rest values of the system's state equal to zero.

In order to optimize the response according to the overshooting $M$ value and the peak time $t_{p}$, they will be used in the reward function. For PID optimization, several reward functions have been developed [62], however, in order to achieve a final performance closer to the requirements of the proposed system, a novel reward function was proposed. The proposed reward function has the following shape:

$$
r_{t}^{s}=C\left(1-\frac{1}{2}\left(\sqrt{\frac{\left(M^{d}-M\right)^{2}}{D}+\frac{\left(t_{p}^{d}-t_{p}\right)^{2}}{W}}\right)\right),
$$

with $M^{d}$ expressing the desired maximum overshoot and $t_{p}^{d}$ the maximum peak time and $A$ will be the gain of the reward and $B$ and $C$ will determine if we prioritize to satisfy the Peak Time or Overshooting constraints [2]. This values are, after some short experimentation, set to $C, D, W=10000,1,40$. This means that reducing the overshooting has chosen to be more important than a fast response.

The final reward is expressed as:

$$
r_{t}=r_{b e s t}-r_{t}^{s}
$$

with $r_{\text {best }}$ the best reward obtained during the learning algorithm and $r_{t}$ the final reward. This is done to accelerate the learning and train the 
network with the improvement of the reward in the time step $t$ and not in the total reward obtained, forcing the learning algorithm to find the more optimal PID gains possible.

The objective is to apply this method twice for each distance, once to tune the parameters of the $\left[\phi, w_{x}\right]$ PIDs' gains and the second one for the $\left[\psi, w_{z}\right]$ PIDs' gains. Then for each of this pairs, the method can be expressed by Alg. 5 .

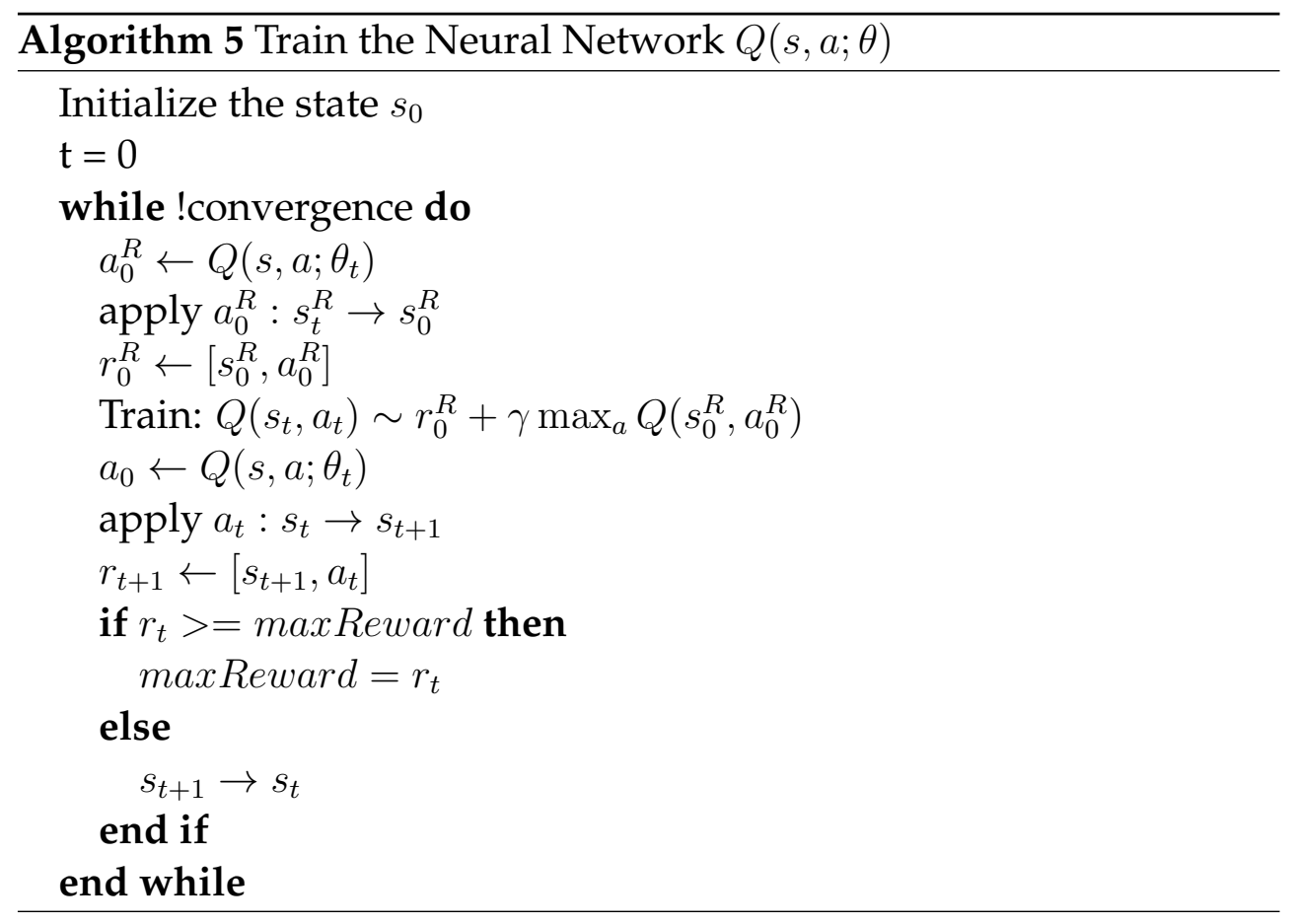

\subsection{Adaptive Control: PID Gains Interpola- tion Using Neural Networks}

The main idea is to design a structure in which, given the estimated the position $P_{i}^{o}$ of a quadcopter, it interpolates the PIDs' gains values from the ones tuned in the previous section. If $P_{i}^{o}$ is expressed in terms of the distances of the quadcopter to the origin of the system $S^{\prime}$ s cooridinates frame, $d_{x}$ and $d_{y}$, the process can be represented as in in Fig. 5.5. In this figure, two different interpolation functions are shown, $f_{x y}$ and $f_{z}$. The function is used to interpolate the gains of the controllers related to the x-axis and y-axis meanwhile the $f_{z}$ is used for the yaw $\psi$ and angular velocity around the z-axis $w_{z}$ controllers' gains. 


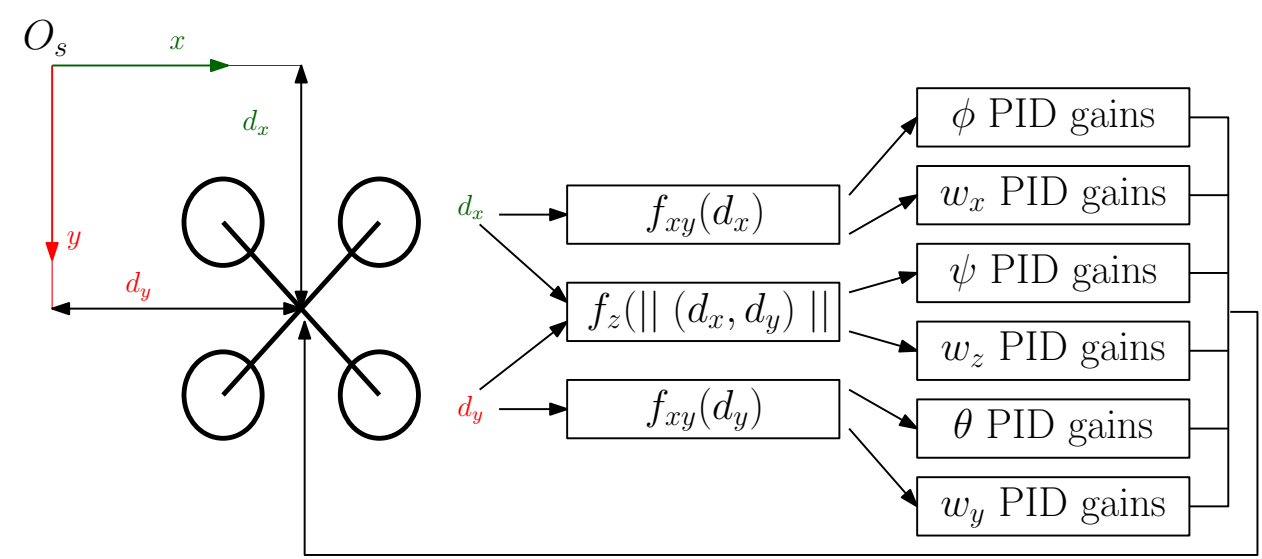

Figure 5.5: Schematic of the adaptive control approach.

It would be possible to already implement an adaptive controller that maps the distance of the quadcopter $i$ to each axis $x$ and $y$-axis and the correspondent calculated optimal PID gains. However, not all the possible distances were considered and some interpolation method has to be applied to fill the estimation of the gains for distances that were not used in the previous step. Depending on the range of possible distances, the gap between to consecutive chosen distances can be important and a regular interpolation method would not work.

Neural Networks have proven to perform optimally in the tasks of function fitting. This property, makes Neural Networks as a perfect option for the current task [39], as the objective is to fit a function that maps the distance to each axis with the PID gains. On the contrary, the structure of this Neural Networks has to be carefully designed as the available data is limited and the Neural Network can be hard to train.

In Section 2.4, the main concepts related with Neural Networks are explained. The chosen structure has 2 hidden layers of 16 hidden units per layer. The input will be the distance, so the input size is 1 . The output layer estimates the PID gains, so it has a length of 6 units. All of the units in each layer are ReLU units. The loss is calculated using the Mean Squared Error (MSE). If $\hat{Y}$ is a vector of $n$ predictions, and $Y$ is the vector of observed values of the variable being predicted, then:

$$
\mathrm{MSE}=\frac{1}{n} \sum_{i=1}^{n}\left(Y_{i}-\hat{Y}_{i}\right)^{2} .
$$

The final structure of the used Neural Network is shown in Fig. 5.6 


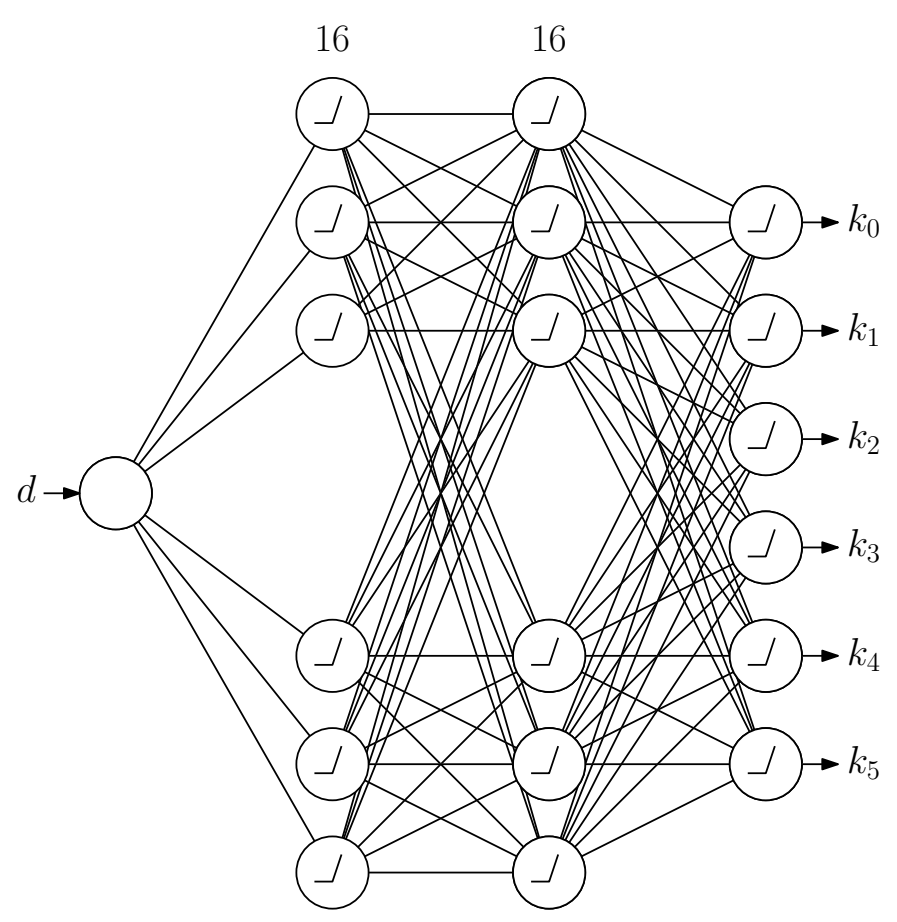

Figure 5.6: Adaptive Control Neural Network structure.

The training is fast $(<5 \mathrm{~min}$.) as the available data is reduced. The implementation and training of the Neural Networks were done using python with Keras library using Tensor-Flow backend.

Two Neural Networks have to be trained for the final Adaptive controller: One for the estimation of the $\left[\phi, \theta, w_{x}, w_{y}\right]$ PIDs' gains $f_{x y}\left(d_{i}\right)$, and another one for the $\left[\psi, w_{z}\right]$ ones $f_{z}(d)$. Once this neural networks are trained, they can be used as in Fig. 5.5 to estimate the value of the gains. After the parameter estimation step has been performed for a certain configuration of the system $S$, the values of $\mathrm{P}_{i}$ can be used to extract the gains for each quadcopters using the already defined interpolation functions, doing:

$$
\begin{aligned}
& {\left[\begin{array}{llllll}
k_{p_{\phi_{i}}} & k_{i_{\phi_{i}}} & k_{d_{\phi_{i}}} & k_{p_{w x_{i}}} & k_{i_{w x_{i}}} & k_{d_{w x_{i}}}
\end{array}\right]=f_{x y}\left(\left|\mathrm{P}_{y_{i}}\right|\right)} \\
& {\left[\begin{array}{llllll}
k_{p_{\alpha_{i}}} & k_{i_{\alpha_{i}}} & k_{d_{\alpha_{i}}} & k_{p_{w y_{i}}} & k_{w_{w y_{i}}} & k_{d_{w y_{i}}}
\end{array}\right]=f_{x y}\left(\left|\mathrm{P}_{x_{i}}\right|\right)} \\
& {\left[\begin{array}{llllll}
k_{p_{\psi_{i}}} & k_{i_{\psi_{i}}} & k_{d_{\psi_{i}}} & k_{p_{w x_{i}}} & k_{i_{w z_{i}}} & k_{d_{w z_{i}}}
\end{array}\right]=f_{z}\left(\mid\left[\begin{array}{ll}
\mathrm{P}_{x_{i}} & \mathrm{P}_{y_{i}}
\end{array}\right]\right)}
\end{aligned}
$$




\section{Chapter 6}

\section{Experimental Setup}

\subsection{Hardware}

\subsubsection{Crazyflie}

Choosing the correct quadcopter for the experimentation was crucial in order to fulfill the goals. The variety of quadcopters nowadays is wide, however, mini-quadcopters are a suitable option as they are easy to handle and experiment with. Within this group of mini-quadcopters, it was decided to work with the Crazyflie 2.0 from Bitcraze [6]. The Crazyflie 2.0 was developed by the Swedish company Bitcraze in 2015 and it has been significantly improved over the last years. Nowadays, the Crazyflie 2.0 is a very competitive drone, with a high flight performance, small size and low price. However, their small size presents important challenges as their low inertia demands fast controllers with low latency. Their battery life is short ( $<7$ minutes) and their maximum payload is one of their bigger weaknesses. However, a main advantage of the Crazyflie 2.0 is that the software is completely opensource, allowing for customizable firmware or API which is a requirement for the implementation of the proposed methods.

\section{Technical Characteristics [10]}

One of the main improvements of the Crazyflie 2.0 with respect to its predecessor is the improved microcontroller. The Crazyflie 2.0 has 2 built-in microcontrollers. The main controller is an ARM embedded processor, the STM32F405 [66]. It runs at $168 \mathrm{MHz}$, and includes a floating point processing unit. The second microcontroller man- 


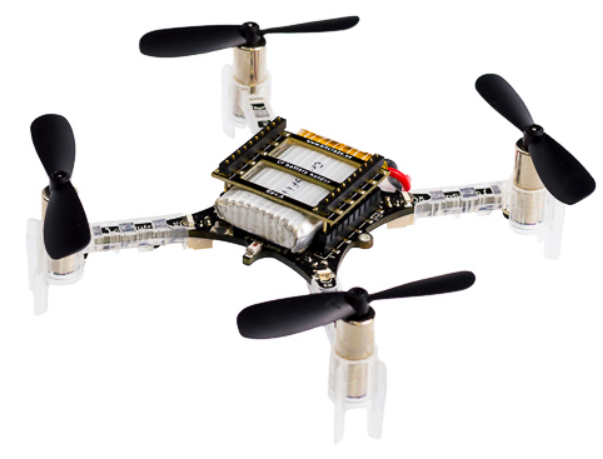

Figure 6.1: The Crazyflie 2.0 by Bitcraze

ages the power management and the radio communication, the ARM nRF51822 [65], that runs at 32MHz.

The common factor in the majority of the Quadcopters in the market is the use of an Inertial Measurement Unit (IMU) to estimate the attitude of the vehicle. In the case of the Crazyflie 2.0, the IMU is a MPU-9250 [31] with a 3-axis gyroscope and a 3-axis accelerometer, to measure the angular velocities and linear accelerations. In the case of the Crazyflie, the IMU axis are set as shown in the Fig. 6.2 with respect to the global coordinate system.

In addition, the Crazyflie incorporates on-board barometer and magnetometer. Both sensor are not used by default inside the sensor fusion algorithms in the firmware.

The Crazyflie 2.0 uses 4 core-less brushed DC motors that achieve a maximum speed of $12000 \mathrm{rpm}$ when the maximum voltage is applied (4.2V). These motors are controlled using PWM signals (See [44]). These PWM values are 16 bits long, and represents the integer values between 0 and 65536. The mapping between this range of PWM values and the RPM output in the motor was obtained empirically in [68], and it follows Eq. 6.1. 


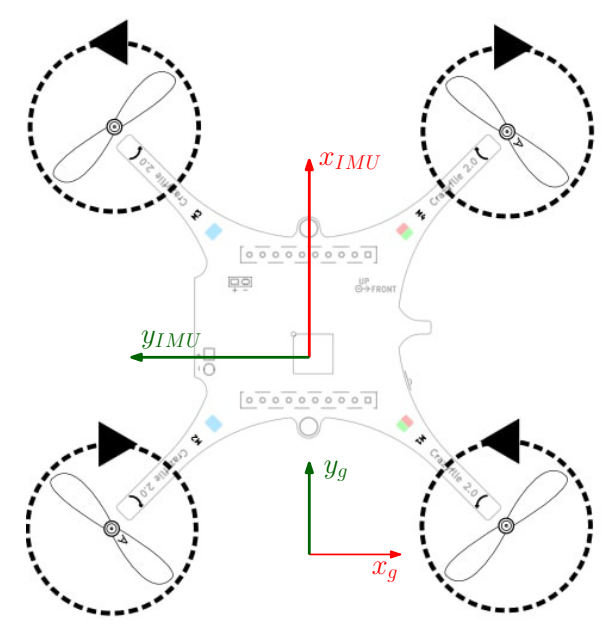

Figure 6.2: $\left[X_{g}, Y_{g}\right]$ represent the global coordinate system and and $\left[X_{\mathrm{IMU}}, Y_{\mathrm{IMU}}\right]$ the on-board coordinate system

$$
\mathrm{RPM}= \begin{cases}0 & \text { if } \mathrm{V}<3.2 \\ 0.2685 \cdot \mathrm{PWM}+4070.3 & \text { if } 3.2 \geq V \leq 4.2\end{cases}
$$

\section{Communication}

Crazyflie 2.0 has an on-board controller dedicated exclusively to power management and radio communication. Bitcraze released their own radio communication system called Crazyradio [8], that incorporates Nordic Semiconductor, nRF24LU1+. It is a USB dongle that allows the user to control the Crazyflie and read its internal values from a computer. Both the Crazyflie and the Crazyradio communicate using a $2.4 \mathrm{GHz}$ ISM band. The Crazyflie and the USB Crazyradio dongle can run up to a transmission rate of $2 \mathrm{Mbs}$. Bitcraze's library [5] and firmware [7] are designed to allow several Crazyflies to be connected to the same Crazyradio USB dongle. All the steps to configure this communications is explained in [10]. 


\section{Physical Parameters}

In order to get both the final dynamical model of a single quadcopter and the system of rigidly attached quadcopters, several parameters of the Crazyflie need to be measured to fullfill the equations developed in Section 2.2 and Section 3. Our model of a single Crazyflie was done using the parameters that were estimated in [21]:

Weight $\rightarrow m_{c f}=27 g$

Propeller's diameter $\rightarrow 23.1348 \mathrm{~mm}$

Arm length $\rightarrow L=46.52 \mathrm{~mm}$

Inertia matrix $\rightarrow I_{C F}=\left(\begin{array}{ccc}16.5717 & 1.2432 & 0.7168 \\ 1.2432 & 26.6556 & 2.0831 \\ 0.7168 & 2.0831 & 29.808\end{array}\right) \cdot 10^{-6} \mathrm{~kg} \cdot \mathrm{m}^{2}$

In Section 2.2, the parameters $C_{T}(n)$ and $C_{P}(n)$ were used and need to be calculated for the Crazyflie. These functions can be approximated as constants and in [45] these values were calculated experimentally for the Crazyflie case:

$$
\begin{gathered}
C_{T}=0.2025 \\
C_{P}=0.11
\end{gathered}
$$

Now, we can use Eq. 6.1 to obtain the values of $T_{i}\left(\mathrm{PWM}_{i}\right)$ and $Q_{i}\left(\mathrm{PWM}_{i}\right)$ depending of the value of the PWM input in each propeller knowing now that the rotations per second $n_{i}$ for the propeller $i$ is:

$$
n_{i}= \begin{cases}0 & \text { if } \mathrm{V}<3.2 \\ \frac{0.2685 \cdot \mathrm{PWM}+4070.3)}{60} & \text { if } 3.2 \geq V \leq 4.2\end{cases}
$$

\subsubsection{Rigid Attachment}

The design of the rigid attachment between each pair of Crazyflies is a very important step to be able to experimentally validate the proposed methods. The stiffness of the structure has to be as high as possible, otherwise, the physical connection parameter estimation step and the 

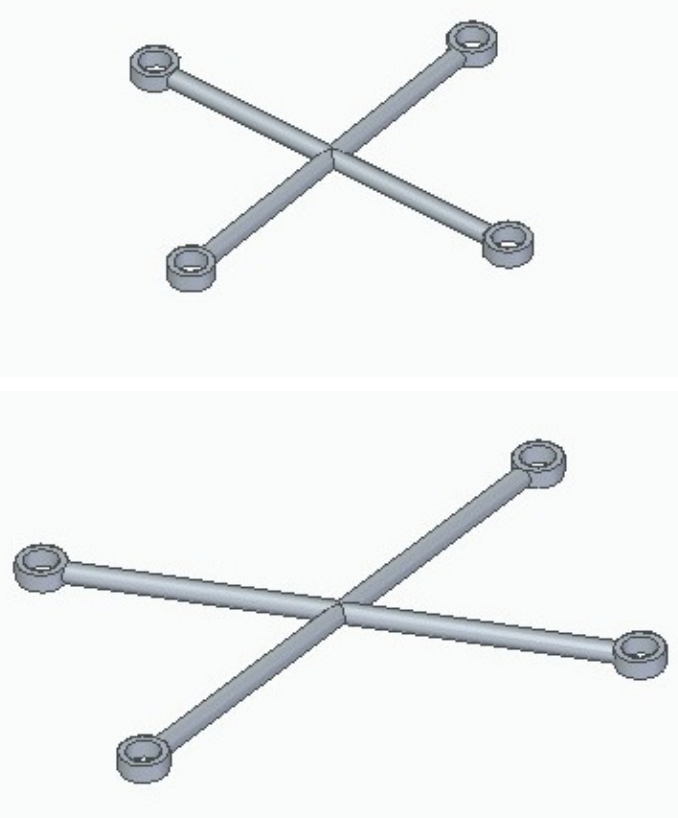

Figure 6.3: 3D design of the 2 rigid attachments used in the experimentation.

final control will show a low performance, as the methods proposed in Chapters 4 and 5 assumed this connection to be rigid. Along with this stiffness, the designing process has to take into account the maximum payload of each Crazyflie ( $15 \mathrm{~g}$ ). There has to be an equilibrium between the stiffness and lightness that in both cases fulfill the requirements. For the current thesis, it was decided to use a conventional 3D printer to produce the attachment as it could fulfill the mechanical requirements, being a cheap, accessible and easy to use technology. For future designs of this attachment, it could be fabricated using innovative materials like carbon fiber allowing to design longer, rigid and lighter structures.

The attachment is shown in Fig. 6.3. The structure is made with hollow tubes to increase the stiffness while reducing the weight. The attachment is done through the motors' structure. In this case, the propellers of the motors need to be detached and reattached in order to add the structure or change its position. In the future, this attachment could be designed using magnets so it is easier to attach or detach the 


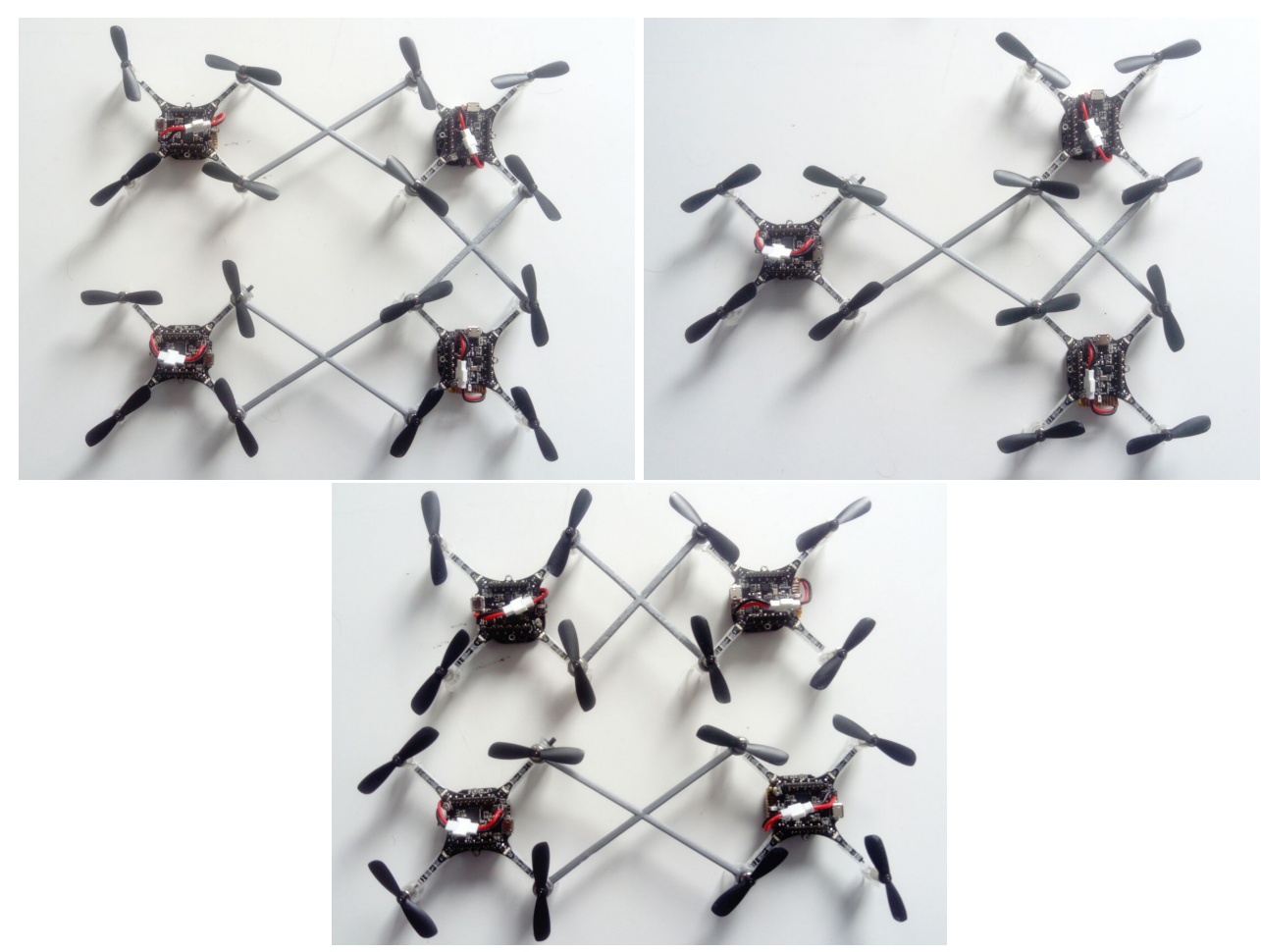

Figure 6.4: Top: Two possible distribution of the system of attached Crazyflies using the designed structures. Bottom: Structure used in the experiments

structure, as in [64].

Even though this first version of the design shown in Fig. 6.3 is very simple, it allows to set the Crazyflies in different configurations, as shown in Fig. 6.4.

Initially, two different attachments have been designed: one with length $6.6 \mathrm{~cm}$ and the other, slightly longer $11 \mathrm{~cm}$ in order to experiment with the Crazyflies using different configurations and lengths. With the current material selection, more that $11 \mathrm{~cm}$ long structures could not guarantee the desired stiffness properties.

\subsection{Global Positioning}

The proposed global positioning approach was described in Section 4.4. For the experimentation, two different localization methods are used: Motion Capture (MOCAP) system and Ultra WideBand (UWB) based Loco Positioning System (LPS) by Bitcraze [9]. All the experi- 


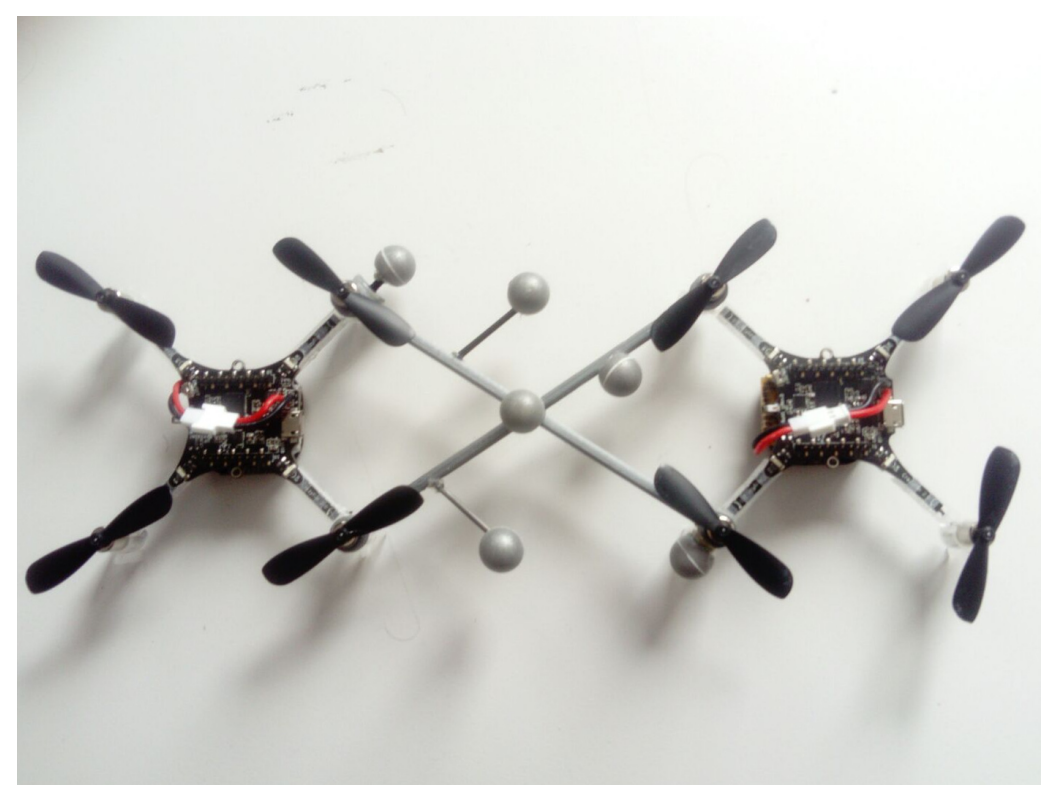

Figure 6.5: Disposition of the MOCAP markers

mentation was done in the Smart Mobility Lab (SML), part of the Integrated Transport and Research Laboratory (SML) in the Royal Institute of Technology $(\mathrm{KTH})$ in Stockholm, Sweden. The flying volume is $6 m \times 6 m \times 3 m$.

\subsubsection{Motion Capture System}

The MOtion CAPture System (MOCAP) designed by Qualisys is installed in the SML lab. It is composed of 12 cameras installed in the ceiling of the lab to cover the entire flying volume. The maximum error in the positioning is given by Qualisys and it is $6 \mathrm{~mm}$. In order to track an object, at least 4 markers has to be attached to it. This tracking is done at a frequency of $100 \mathrm{~Hz}$. For the experimentation, the MOCAP system is used to track the position of the system of attached Crazyflies. The markers are set in the system of attached Crazyflies as shown in Fig. 6.5 .

It is important to notice, that the markers are set in the connecting structures, not in the Crazyflies, therefore, the MOCAP system does not give any information about the distance of relative orientation between the Crazyflies. This parameters has to be estimated.

Then, this estimated position will be used in parallel in two tasks. First, it will be used as the ground truth of the actual position of the 
system $S$, in order to be compared with the individual estimation of each Crazyflie. Besides, this measurements will be used as the main localization method for the system of attached Crazyflies for a subset of experiments.

\subsubsection{Loco Positioning System}

The Loco Positioning System (LPS) is a UWB-based indoor localization system developed by Bitcraze, based on Decawave DWM1000 microchip [14]. UWB is a radio technology used for short-range, highbandwidth $(<500 \mathrm{MHz})$ communications. One of the more spread usages of this technology is indoor tracking applications.

The LPS system was designed for 3D position indoor tracking of the Crazyflies. It is composed by at least 6 anchors, and one LPS deck that is connected to the Crazyflie. The idea is simple: by sending short high frequency radio messages between the anchors and the LPS deck, the system measures the distances between the deck and the anchors, estimating the position of the Crazyflie. To accurately estimate the real time position of a Crazyflie, the exact position of the anchors must be measured. The precision of this measurement will affect the final precision of the system. Besides, the anchors have to be distributed over the available space, following the guidelines proposed by Bitcraze [9].

\subsection{Software}

For the experimental setup, the software implementations and modifications were crucial. First, the simulator was developed, as all the proposed methods needed to be validated in simulation before implementing them in the real system. Once the simulation validation was done, it was necessary to modify the firmware developed by Bitcraze to perform the main steps already described. Finally, a GUI was developed to control the real system of attached Crazyflies.

\subsubsection{Simulator and User Interface Application}

\section{Simulator}

The objective was to develop a quadcopter simulator that could be readapted to any other quadcopter in which all the physical parameters, 


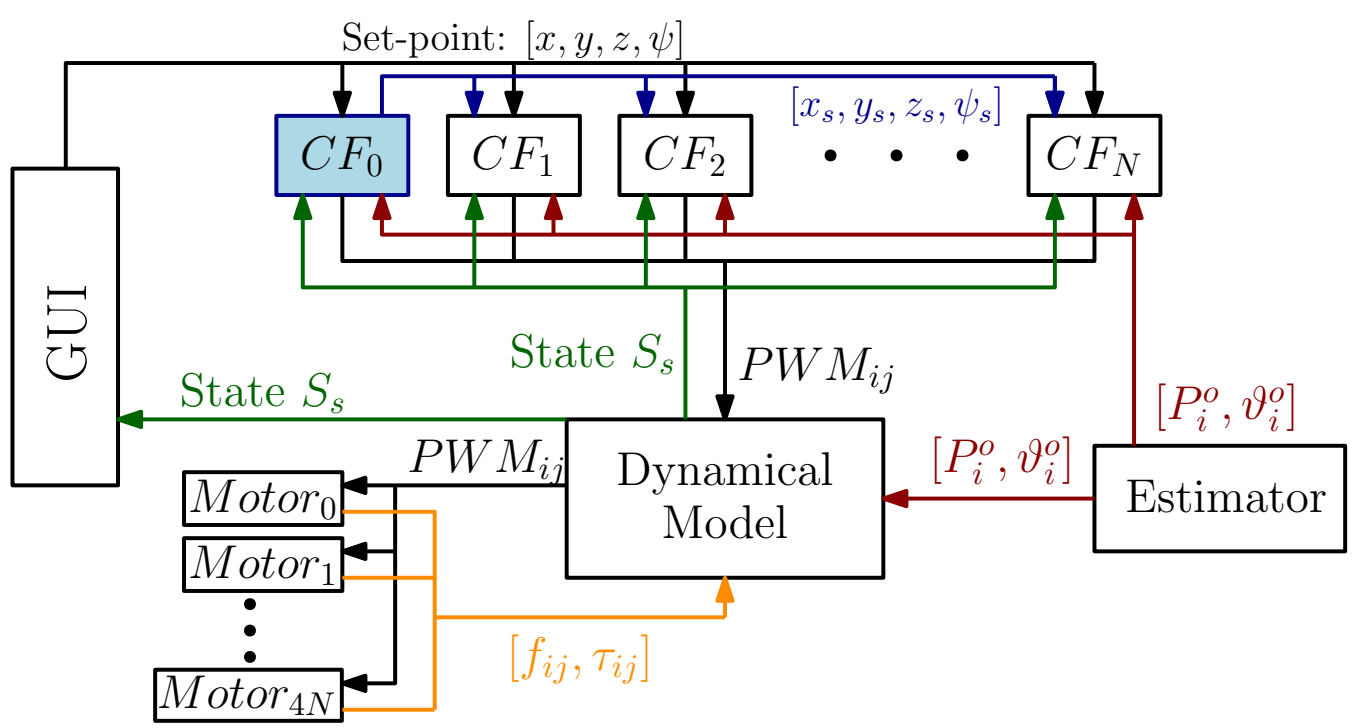

Figure 6.6: Simulator's backend structure. $C F_{i}$ represent each of the blocks that simulate the individual measurements from the Crazyflies' IMUs and will run the PID controllers. $C F_{0}$ is the leader CF that sends its estimation of the system position to the rest of Crazyflies. $P W M_{i j}$ is the PWM that needs to be applied to every motor. $\left[f_{i j}, \tau_{i j}\right]$ represents the linear force and torque applied by each motor. $S_{s}$ is the actual state of the system $S$.

both of the quadcopter and the attachments, are easy to modify. There are several simulation environments and programming languages that could have been chosen for the purpose. However, the API for the Crazyflie was developed in Python by Bitcraze. Therefore, the simulator was developed in Python to allow code re-usage in the final implementation of the GUI to control the real system of attached Crazyflie.

An easy-to-use GUI interface for the simulator was implemented, in which the behavior of the system of quadcopters is shown in realtime. The inner architecture of the simulator is shown in Fig. 6.6.

With the GUI it is possible to easily change the position, rotation and number of the quadcopters within the system $S$. Also, it is possible to select the value of the variances for the biases and noises described in Eqs. 3.22 and 3.23. Last, it is possible to choose the position set-points of the system in real-time. After any simulation, the user can plot and save the positions traced by the system. In Fig. 6.7 is shown the final aspect of the simulator.

It was important to simulate the communication between the quad- 


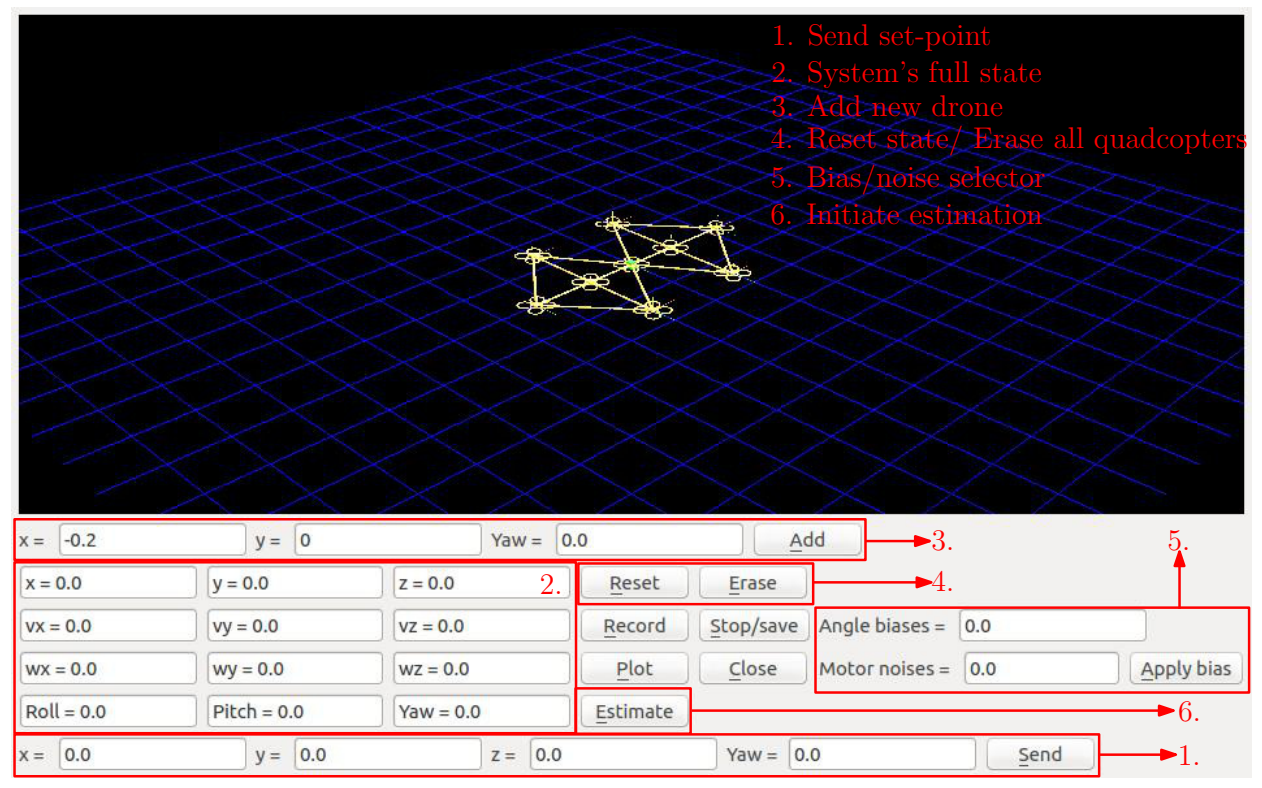

Figure 6.7: Main GUI's features.

copters, the measurement of each individual IMU and the individual control approach of the entire system of attached quadcopters. Therefore, the simulator was designed in different blocks, as shown in Fig. 6.6 .

Within the simulator, the user can choose between 3 different modes: estimation mode, real physical parameters flight and estimated parameters flight. In the estimation mode, the system of quadcopters perform, step by step, the operations described in Section 4 . Then, in both modes real physical parameters flight and estimated parameters flight, the flight of the system $S$ is controlled by the user sending the desired set-points. The only difference is that while in real physical parameters flight, the dynamical model of the system, and the individual IMUs measurements, use the actual parameters of the system, while in estimated parameters flight the estimated parameters by the estimation mode are used.

\section{User Interface Application}

A simple GUI was developed to control the real system, as it allows to change different parameters or send commands in real time. The simplified GUI architecture is shown in Fig. 6.8

The GUI automatically detects all the available Crazyflies and connect to them by just pressing a button. Once they are connected, the 


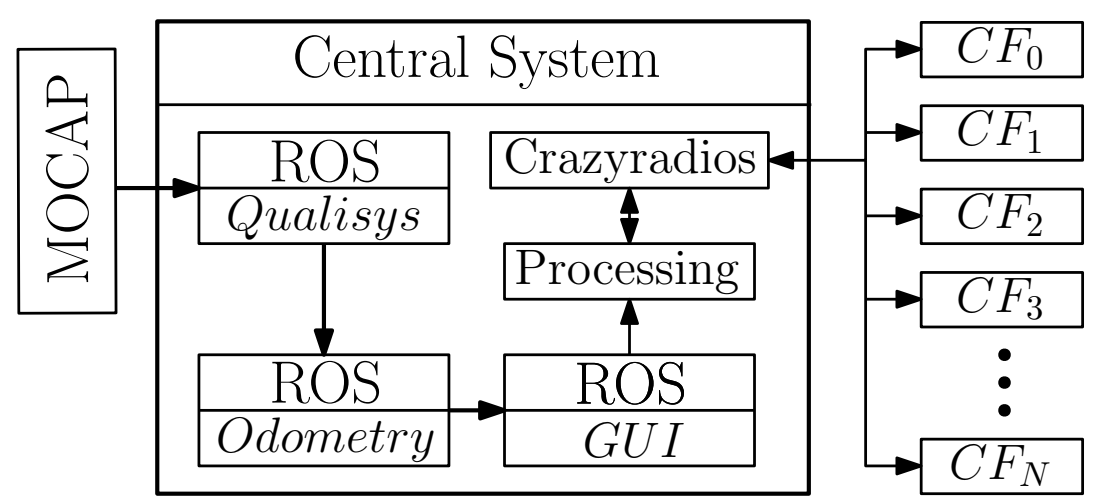

Figure 6.8: Simplified architecture of the final implementation of the system.

battery levels of each Crazyflie are displayed. There are to possible options: perform the parameter estimation of the system, or add the real values of this parameters manually. This was done to perform flights with the real and the estimated parameters to compare them afterwards. Then, the user only needs to choose between one of the trajectories shown in Fig. 6.9 that were implemented for the GUI. The position estimation of the leader Crazyflie is shown in real-time in the GUI. Besides, the GUI received the measurements of the MOCAP and LPS system and also shows these values in real-time.

The GUI allows to record the data from the MOCAP and the individual position estimations when desired and stored it later. This was included as this data needs to be analyzed later to prove the validation of the proposed methods. The GUI was implemented in python as a ROS node [60], to allow the communication with the MOCAP system.

The GUI is shown in Fig. 6.10. Inside the GUI, the battery level of each Crazyflie is obtained and filtered so that, if the battery level of any of them is critical, the system enters in emergency mode and is automatically sent to the initial point, usually $[0,0,0]$.

The equations described in Section 4.3 were implemented in the GUI, so the main parameters are estimated by the GUI's backend. After the estimation, the GUI automatically sends these parameters to each Crazyflie and set them ready to start the flight. During the flights, the GUI sends the set points of the chosen trajectories while reading the position estimation of the leader Crazyflie. This estimation is read and sent to the rest of Crazyflies at a rate of $100 \mathrm{~Hz}$, same as the PID position controllers inside the firmware. 

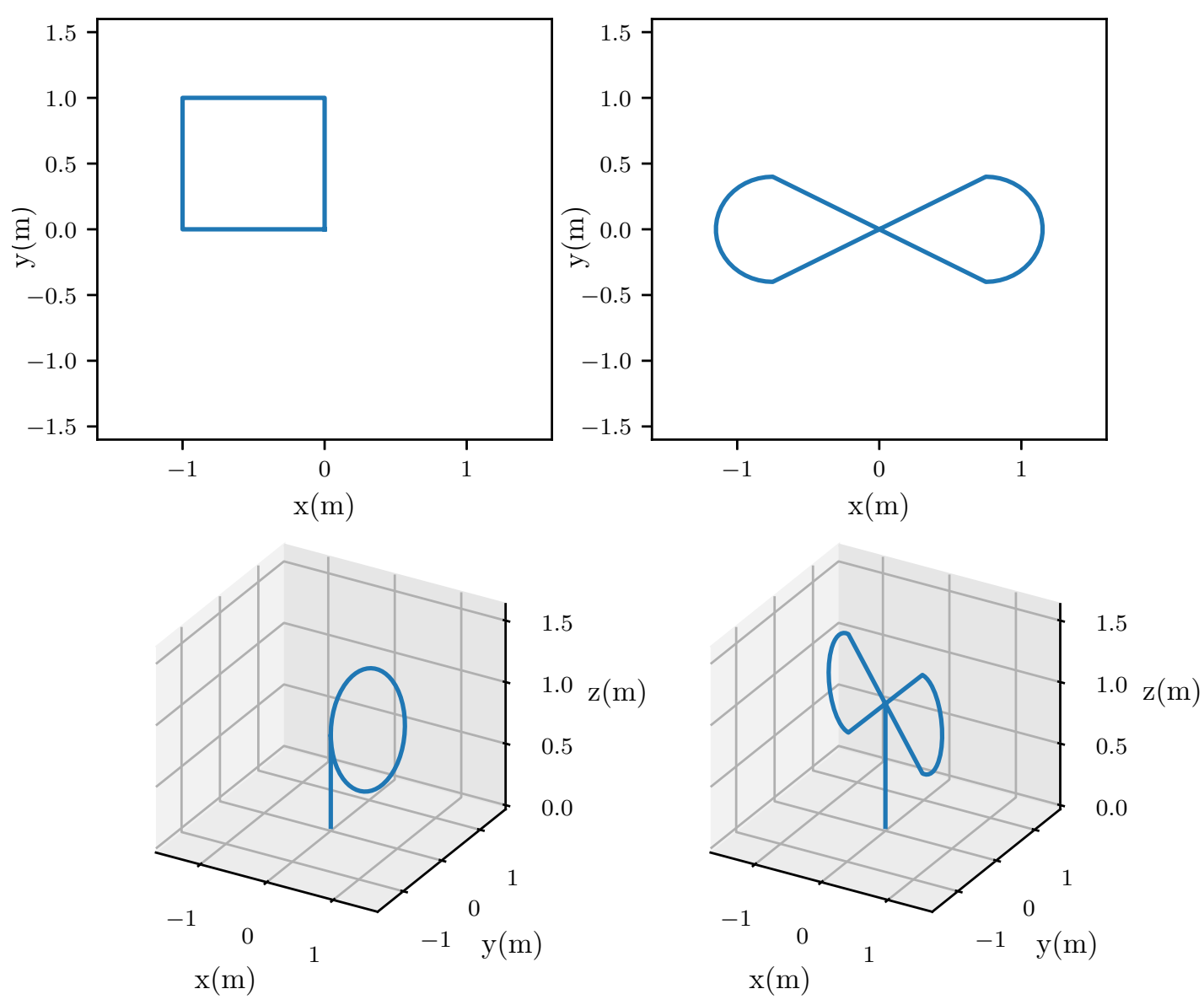

Figure 6.9: Implemented trajectories that can be chosen by the user. Top: xy view of trajectories, in which the height is $1 \mathrm{~m}$. Top left: $2 \mathrm{D}-$ square. Top right: 2D-eight. Bottom: 3D trajectories, which objective is to produce displacement of the system in the 3 axis simultaneously. Bottom left: 3D circle. Bottom right: 3D-eight

The system is composed by a set of Crazyflies, a Crazyradio USB dongle for each group of 4 Crazyflies, the ground station, the MOCAP system, and any other positioning system that may be added. The ground station links all these agents as there is no direct communication between the Crazyflies or between the MOCAP system and the 


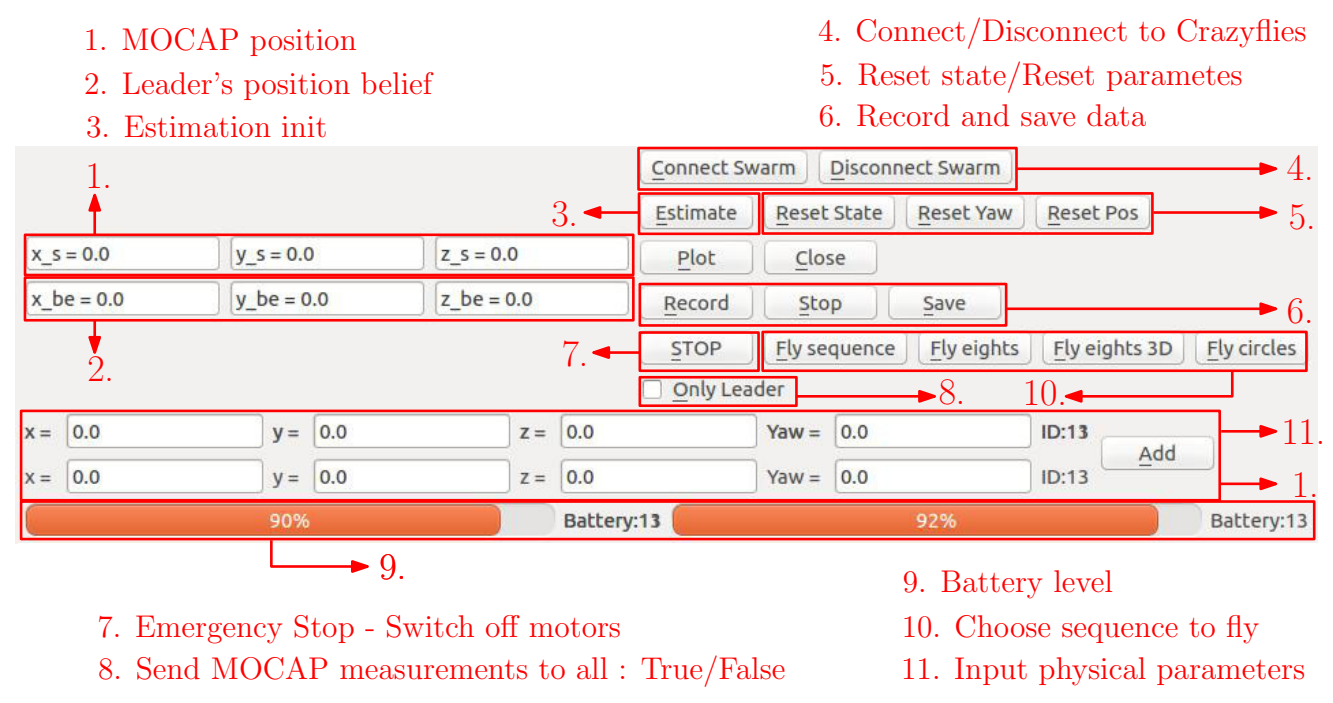

Figure 6.10: Simplified structure of the implemented custom firmware for the Crazyflie.

\section{Crazyflies.}

The communication between the Crazyflies and the ground station is simple and is done through the Crazyradio USB dongle. The physical characteristics of this communication is explained in Section 6.1.1. The API automatically handles the communication, including the reading and writing of the variables inside the firmware, or connecting automatically to a swarm of Crazyflies. It also allows to send set-points in parallel to all the Crazyflies, a basic feature in order to send trajectories or any other command to the system of Crazyflies.

The communication between the Crazyflies is also handled by the ground-station through the Crazyradio. The ground station receives information from the set of Crazyflies and decides which information or commands to send to each of them.

The MOCAP system installed in the SML lab handling is done using a ROS node that publish messages with the pose of all the detected objects every $10 \mathrm{~ms}$. In order to receive this data, two nodes have to be initialized in the ground station. One that subscribes to this messages (qualisys node) and another one, with the ID being the name of the object inside Qualisys' MOCAP's software that is going to be tracked. This last node subscribes to qualisys node, transforming the desired message to an odometry type message that allows to read the pose, time-stamp and other information from the object at a rate of $100 \mathrm{~Hz}$. 


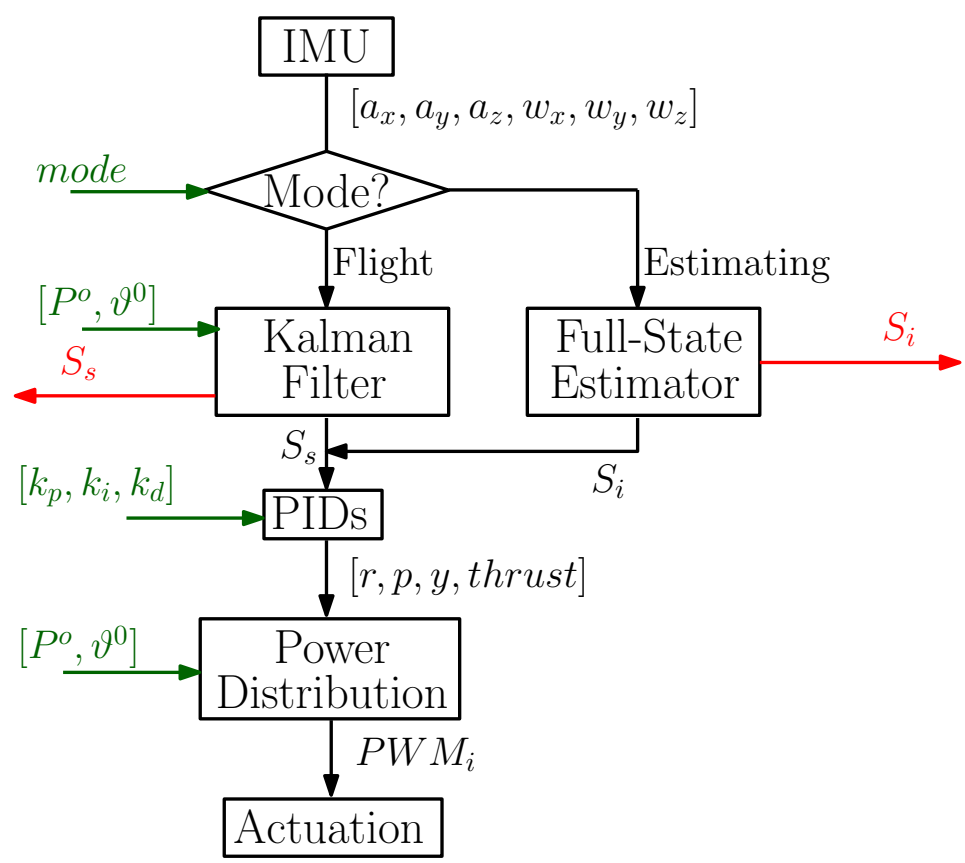

Figure 6.11: Simplified structure of the implemented custom firmware for the Crazyflie. In green, variable values set by the Central Computer. In red, current state estimated by the firmware sent to the Central Computer

Finally, the GUI is set as a ROS node that subscribes to the odometry node getting the data measured by the MOCAP. As it was already mentioned, this data can be stored to be processed later.

\subsubsection{Crazyflie's Firmware Setup}

Crazyflie's firmware [7], developed by Bitcraze, was designed to optimize the flight of a single Crazyflie, including a huge amount of features: communication, a Kalman Filter, inner variables values read and write in real time, such as the PID gains, or the state estimation, or a full-state PID controller. However, to allow the implementation of the proposed method in the current thesis most of these features need to be modified. The firmware is implemented in C and is open-source. The simplified structure is shown in Fig. 6.11 


\section{Physical Parameter Estimation: Full-state Estimator}

In Section 4 the equations for the full-state estimator are explained. To allow the correct physical parameter estimation these equations and methods need to be implemented inside the firmware. The equations implemented in the firmware are exactly the ones expressed in Eqs. 4.5 and 4.6 . The algorithm to detect whether the Crazyflie is moving or not has to be implemented in the firmware (see Alg. 2).

For this thesis, the firmware was modified, allowing to switch between the Kalman filter and the custom full-state estimator easily, from the ground station GUI program. This way, the system could start flying right after the physical parameters were estimated, without needing to restart the system.

\section{Sending Physical Parameters to the Crazyflie: KF Modification}

The firmware already implemented a method to read and write most all the inner variables needed by just adding this variables to the LOG (read) or PARAM (write) lists inside the firmware. Then, the variables of the position $P_{o}$ and rotation $\vartheta$ of the Crazyflie relative to the system $S$ coordinate frame were added to the Kalman filter, and included in the PARAM list to allow their real-time modification from the GUI. This way, the estimated parameters could be sent to each Crazyflie after the estimation process was done.

These data has to be used by the Kalman filter to predict the system's state in each Crazyflie. Then, the dynamic model inside the Kalman filter was modified. The original equations of this model were not changed, but the the position, velocities, attitude and angular velocities were rotated and translated according to the estimated position $P_{o}$ and rotation $\vartheta$ of the Crazyflie relative to the system $S$.

The input of the Kalman filter are the measurements obtained from the accelerometer and gyroscope. Due to the modifications, expressed in Eq. 4.43 and 4.49 , the output is the full-state, position, linear velocities, angular velocities and attitude of the system $S$. The matrices expressed in Eq. 2.42 are kept unchanged from the ones implemented originally in the firmware [7]. 


\section{PID Controller Modification}

Only the Power distribution block, explained in Chapter 5, was modified, implementing Eq. 5.5. This block was in charge of mapping the estimated needed thrust and $x, y, z$ moments to the PWM values to be applied in each motor. As it was explained in Section 3 this block needs also to be fed with the estimated physical parameters. Then, the needed writable variables were also added to this block, so the parameters could be sent. The variables to change the PID gains inside the firmware in real time were already implemented, no modifications were needed.

\subsection{Architecture overview}

In Fig. 6.12, the final architecture's overview of the system is represented. In this figure, all the communications are represented by arrows, and different colors have been used to represent the different communication channels.

To validate the proposed localization proposed solution, two different localization methods are used: MOCAP system and LPS system. In the MOCAP case, the position measured will be sent to one of the Crazyflies that will update its estimation at a rate of $100 \mathrm{~Hz}$ and send this estimation back to the ground station in each time step. The ground station will then spread this estimation to the rest of the Crazyflies attached to the system $S$ that will update their estimation. This procedure is only done if the system is not using the Loco Positioning System. Ideally, the estimation of the leader Crazyflie would be sent directly to the rest of the Crazyflies, but the communication between Crazyflies has not been developed yet. However, during the experimentation, it is shown that this small increment in the latency does not affect the final performance of the system.

In the LPS case, it was configured to be used within the flight volume available in the SML lab. The main objective is to validate the global localization solution proposed in the current thesis. As a consequence, only one of the Crazyflies, the leader, will use the LPS deck. Then, the position estimation of the leader will be spread to the other Crazyflies through the Central Computer via the Crazyradio. The idea is to prove that the latency in this process is low enough to ensure system stability. During the experiments performed with the LPS system, 


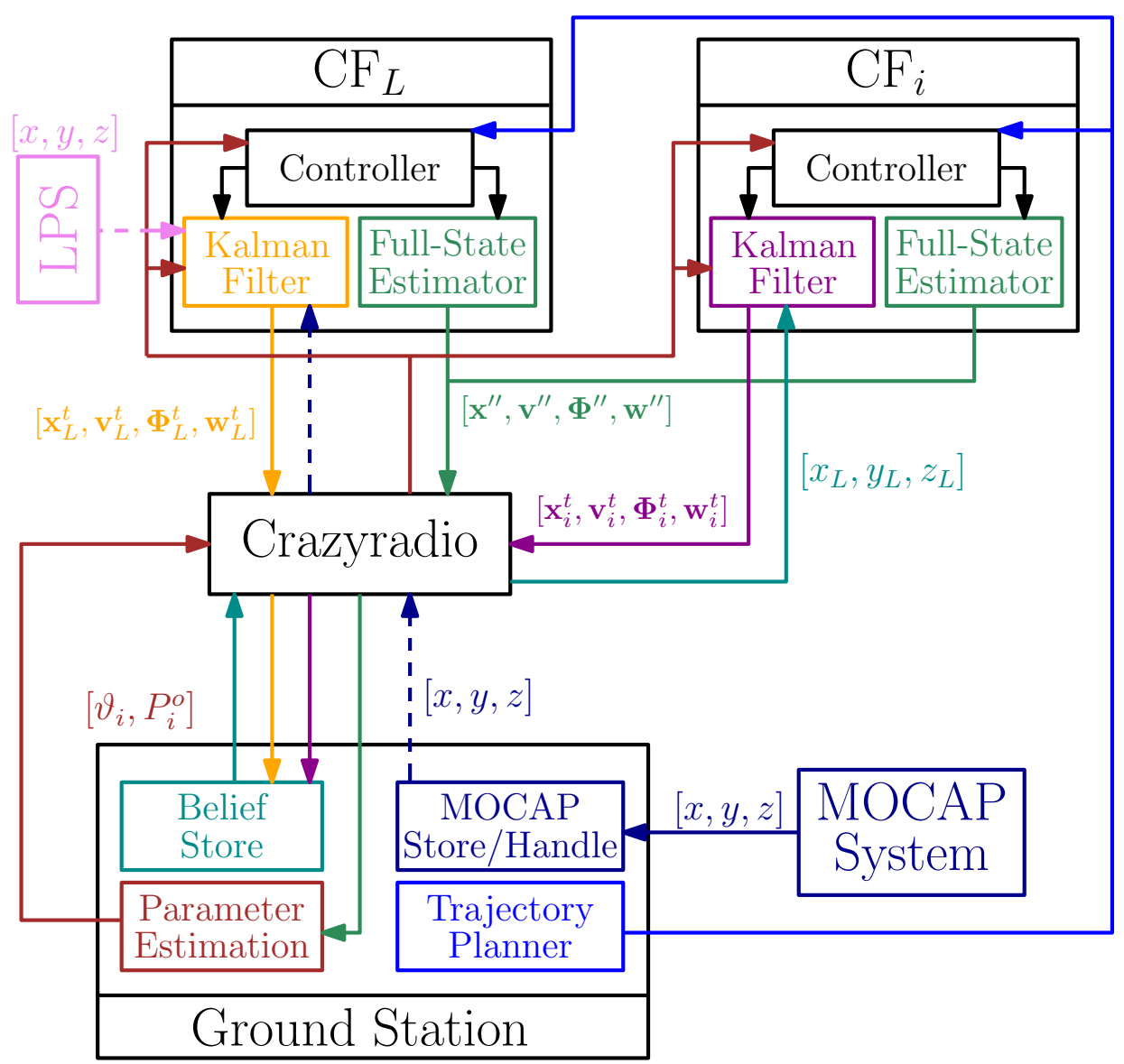

Figure 6.12: Representation of the final architecture of the system. The slaves Crazyflie are represented by the $i$. The leader Crazyflie is named by $\mathrm{CF}_{L}$. The dash arrows in the LPS blocks mean that this communication only happens when the Leader Crazyflie has a LPS deck connected. Then, the MOCAP measurements are not sent to the any Crazyflie.

the MOCAP will be used only as a ground truth comparison, but its measurements are not sent to any of the Crazyflies. 


\section{Chapter 7}

\section{Results}

\subsection{Description of Experiments}

The purpose of the experimentation is to validate the methods proposed in Chapters 4 and 5 . This experimentation was done both in simulation and using the Crazyflies. The experiments designed are similar for both the simulated and the actual system. For all the experiments done in simulation, white noise was added to the measurements of the angular velocities, proportional to the ones experimentally observed in the real IMU measurements in order to bring the simulated model closer to the reality. The experiments were divided in:

- Parameter Estimation: validation of the physical parameters estimation approach. The parameters to be identified are the rigid connection parameters: distances $d_{i j}$ and absolute rotations around the z-axis $\vartheta_{i}^{o}$, as the position $P_{i}^{o}$ of each Crazyflie can be derived from this parameters, see Section 4.3.4.

- Flight Control and Stability: The objective was to validate the proposed control architecture showing that the system of attached Crazyflies and the simulator can perform stable flights while following a trajectory. For this set of experiments, the true physical connection parameters were used.

- Full approach: in this last set of experiments, the fusion of both the Parameter Estimation and Flight Control steps was tested. The goal was to study how much would the erroneous estimations affect the system's stability. 


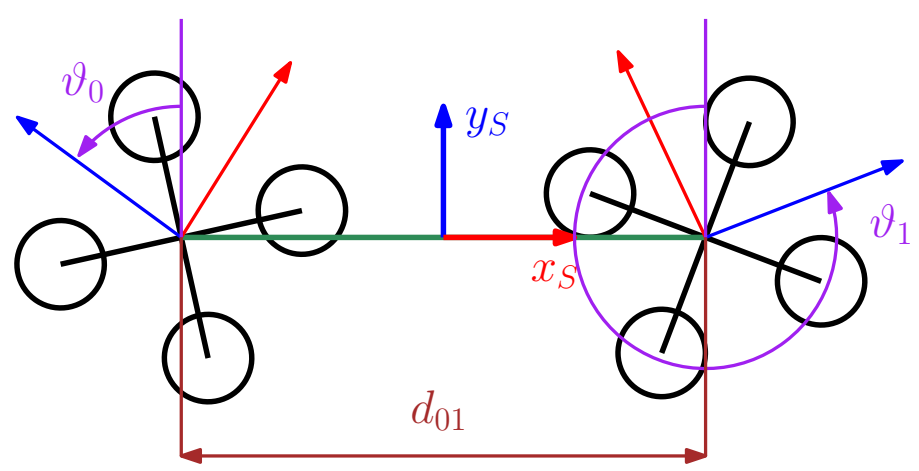

Figure 7.1: Generic distribution with the main parameters that were estimated.

\subsubsection{Parameter Estimation}

The experiment designed for the parameter estimation validation is simple: run the experiments 10 times for each possible combination of distances $d_{i j}$ and different rotations around the z-axis $\vartheta_{i}$ for the two quadcopter case system, see Fig. 7.1. The reason why only two quadcopters were used is because it is the simplest case which and easier to analyze. As it is mentioned in Section 4.3. only the distance $d_{i j}$ between each pair of quadcopters and each individual relative rotations around the z-axis $\vartheta_{i}$ are needed to estimate the positions $P_{i}^{o}$ of each quadcopter in the $\mathrm{N}>2$ case. The obtained errors, along with the mean and variances would give a deeper insight of the quality of the approach.

To evaluate and compare the estimation error during the experiments, the Root Mean Square Error (RMSE) was used. This indicator was chosen instead the MSE as the RMSE is expressed in the same units as the parameter that is being measured. The RMSE is defined as:

$$
R M S E=\sqrt{\frac{1}{n} \sum_{n}^{i=1}\left(Y_{i}-\hat{Y}_{i}\right)^{2}}
$$

where in this case, $Y_{i}$ represents the trajectory coordinates and $\hat{Y}_{i}$ the traced position of the system. 


\subsubsection{Control Architecture and Strategy}

For the second group of experiments, three trajectories were used, from the ones shown in Fig. 6.9 the 1 meter 2D-square, the 2D-eights and the 3D-circle. The 2D-square trajectory was designed to excite only one of the $x, y$ and $z$ position controllers at the same time. The 2D-eight trajectory was implemented to force the system to excite both the $x$ and $y$ position controllers. It tests how the perturbations in one axis may affect the other. Finally, the 3D-circles, excite the three axis at the same time. Performing this kind of trajectories is crucial for the validation of a quadcopter's controller. It may happen that a fast response in one of the axis may unstabilize the entire system. All these trajectories are run for the following values of $\vartheta_{0}, \vartheta_{1}$ and $d_{01}$ :

\begin{tabular}{|c|c|c|}
\hline$d_{01}$ & $\vartheta_{0}$ & $\vartheta_{1}$ \\
\hline $154 m m$ & $0^{\circ}$ & $0^{\circ}$ \\
$154 m m$ & $180^{\circ}$ & $0^{\circ}$ \\
$154 m m$ & $180^{\circ}$ & $270^{\circ}$ \\
$154 m m$ & $270^{\circ}$ & $90^{\circ}$ \\
\hline $132 m m$ & $0^{\circ}$ & $0^{\circ}$ \\
$132 m m$ & $180^{\circ}$ & $0^{\circ}$ \\
$132 \mathrm{~mm}$ & $180^{\circ}$ & $270^{\circ}$ \\
$132 \mathrm{~mm}$ & $270^{\circ}$ & $90^{\circ}$ \\
\hline
\end{tabular}

Table 7.1: Combination of values of $\vartheta_{0}, \vartheta_{1}$ and $d_{01}$ that were used in the experiments.

The experiments in this section were different for the simulation and experimental case.

To evaluate and compare the positioning error during the flights, the Mean Square Error (MSE) was used. The MSE is defined as:

$$
M S E=\frac{1}{n} \sum_{n}^{i=1}\left(Y_{i}-\hat{Y}_{i}\right)^{2}
$$

where in this case, $Y_{i}$ represents the trajectory coordinates and $\hat{Y}_{i}$ the traced position of the system. 

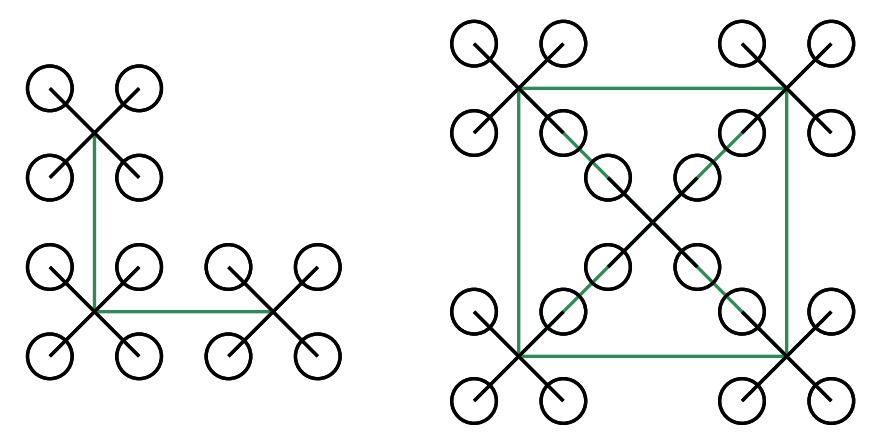

Figure 7.2: $N>2$ quadcopters distributions used in the experimentation

\section{Simulation}

As it was mentioned before, three different trajectories were designed for this step of the experimentation. In simulation, the 3D-eight trajectory is also used (see Fig. 6.9). The idea was to validate the proposed controller and architecture separately from the parameter estimation approach.

For the first group of experiments, all the designed trajectories were applied to all the possible distributions of $d_{i j}$ and $\vartheta_{i}^{o}$. As a consequence, a total of sixty flights were performed per set. Is not possible to plot the measured data for each flight in the thesis, therefore, a selection of them is shown.

After some of these trajectories has been plotted, all the stored data was used to analyze the evolution of the error in the different configurations. Besides, the simulator was designed to send the position measured in the leader quadcopter to the rest of quadcopters with a constant delay of $10 \mathrm{~ms}$. This was done to simulate the real latency that would appear in the real system when applying the architecture proposed in Section 4.4 .

To test the stability with a greater number of quadcopters, two systems of three and five quadcopters were used. These chosen distributions were all symmetric, see Fig. 7.2. Some of the obtained trajectories are plotted.

\section{Real system}

The approach was identical to the simulation case. To fully validate the proposed methods in the real system, the experimentation was more 
specific, subdividing the experimentation in more groups. In this case, only three trajectories were used, the 2D-Square, the 2D-Eight and the 3D-Circles, see Fig. 6.9. Only two quadcopters were used for the experiments carried on the real system, as it is the simplest case, being otherwise hard to analyze the results. The 2 structures designed for the experiments depicted in Section 6.1.2 were used, having two different distances between the Crazyflies.

The system stability was tested using both the MOCAP and LPS systems separately. In the case of the MOCAP experimentation, the measurements were to one of the Crazyflies, chosen randomly at the initialization of the GUI, and the belief in the position of this leader Crazyflie was spread to the other Crazyflie via the central computer's Crazyradio in real time. In the case of the LPS experimentation, the MOCAP measurements were not sent to any ot he Crazyflies. On the contrary, one of the Crazyflies, the leader $L$, had the LPS deck attached, and therefore, it received the tracking estimation from the LPS. The belief of this Crazyflie was then sent to the other Crazyflie, the slave $s$. The objective of doing this was to analyze the stability with a high precision positioning system (MOCAP) and a low precision one (LPS).

In this set of experiments, the real values of $d_{i j}, \vartheta_{i}$ were not estimated but sent to them. In both cases, the MOCAP measurements were stored to be used as the ground truth for comparison. The used distributions are shown in Table 7.1. It was also experimented with a single Crazyflie with the original firmware in order to have comparison reference for each proposed trajectory.

To have a better insight of the distribution of the obtained errors, the MSE was calculated in 8 separated groups. These groups correspond to the 8 distributions written in Table 7.1. The MSE values were calculated over the MOCAP measurements directly, the objective was to compare the actual position of the system with the desired trajectory.

\subsubsection{Full approach}

The validation of the full approach is only achieved in the real system as the estimation error is smaller in the simulation case, being the real system, the worst case scenario. The approach was the same as in the previous sections, however, now the estimation step was performed before each experiment, and the estimated parameters were sent to 


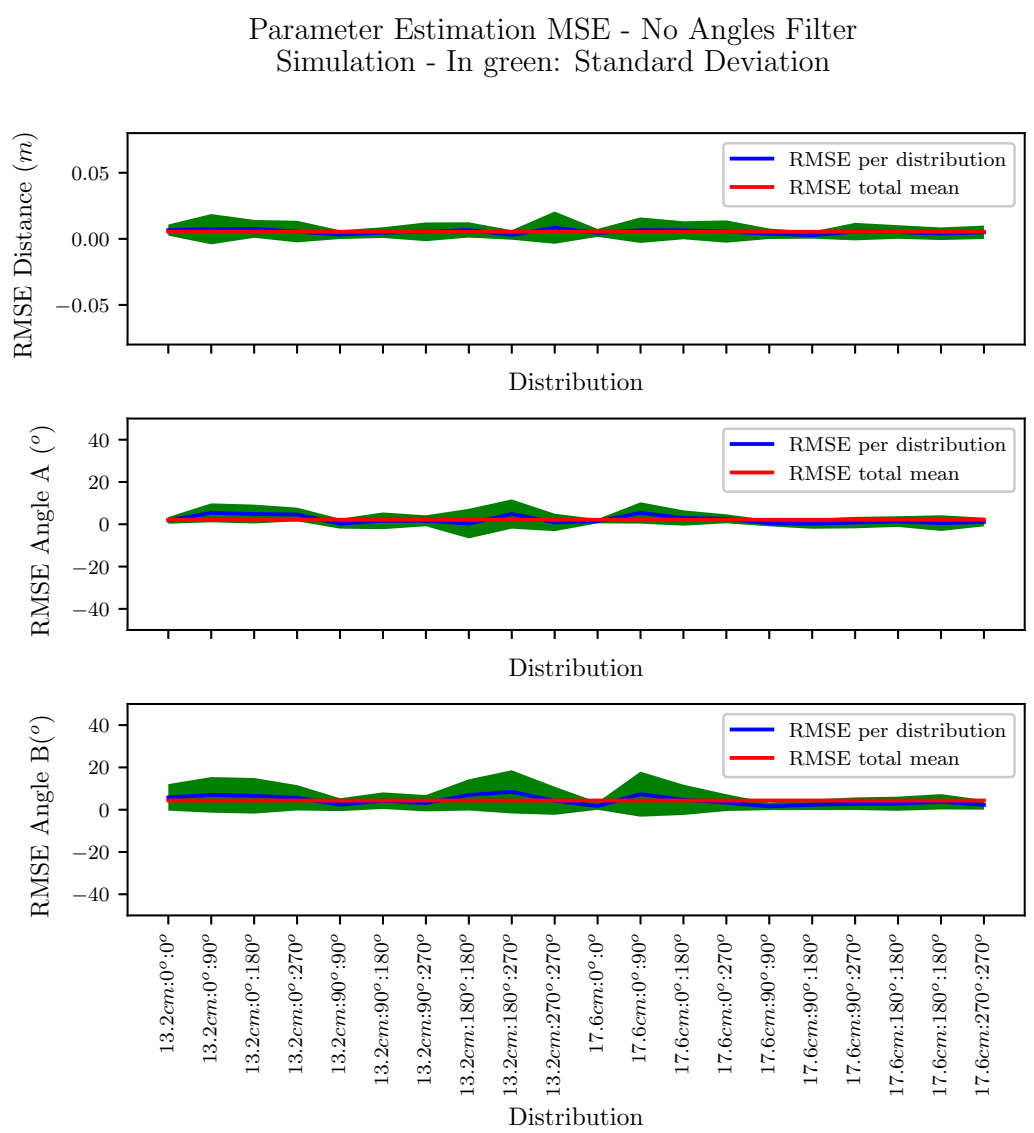

Figure 7.3: RMSE of the estimated physical parameters depending of the physical distribution in simulation. In green, the standard deviation of each RMSE.

each Crazyflie. The parameters were estimated for eight different configurations, written in Table 7.1, and then the three trajectories were applied with the estimated parameters. In this case, only the MOCAP measurements were used, and the measurements were still only sent to the leader $L$.

\subsection{Simulation Results}

\subsubsection{Parameter Estimation}

In Fig. 7.3 the values of the RMSE value per distribution are plotted after running the estimation method 10 times with each distribution. 
Parameter Estimation MSE - Angles Filter

Simulation - In green: Standard Deviation
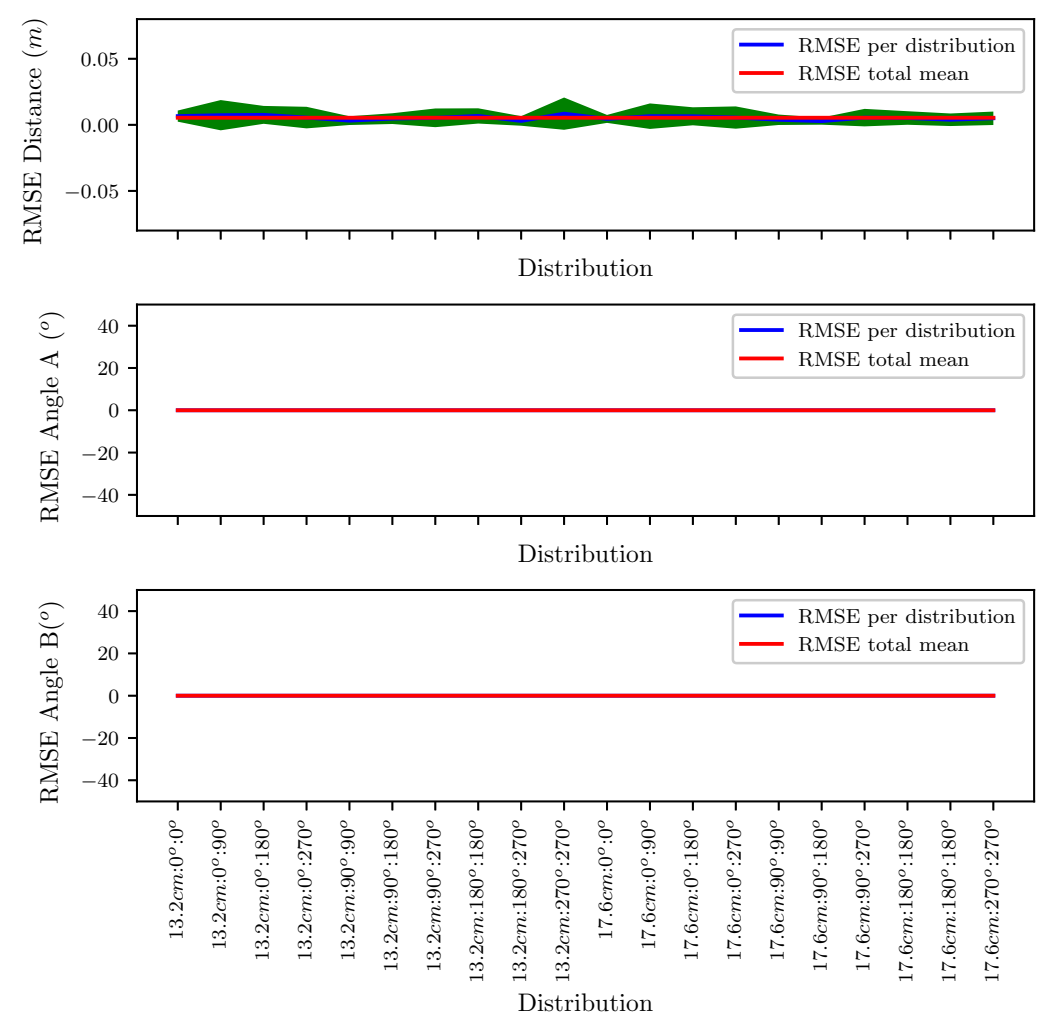

Figure 7.4: RMSE of the estimated physical parameters depending of the physical distribution after the angles has been rounded to the closer normalized value in simulation. In green, the standard deviation of each RMSE.

It can be observed that the obtained errors are small $(<5 \mathrm{~mm}$ and $<5$ degrees). However, if one assumes that the rotations around the $\mathrm{z}$-axis are fixed, which is the case for the structure used in this thesis, one can use this information to further reduce this error. These values are $0^{\circ}, 90^{\circ}, 180^{\circ}$ and $270^{\circ}$. If the estimated angles are rounded to these values depending on their proximity to them, then a new RMSE distribution is obtained, plotted in Fig. 7.4

In Fig. 7.4 it can also be observed that the RMSE value decreases to zero, meaning this that in every case the estimated angles were correctly classified. 


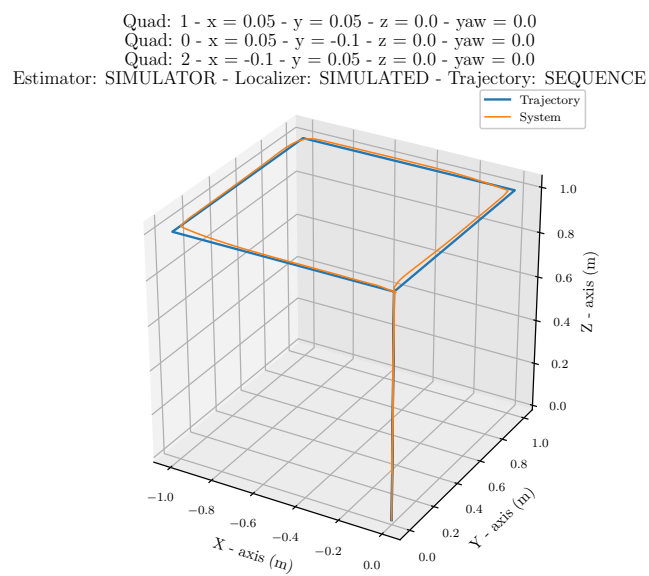

(a)

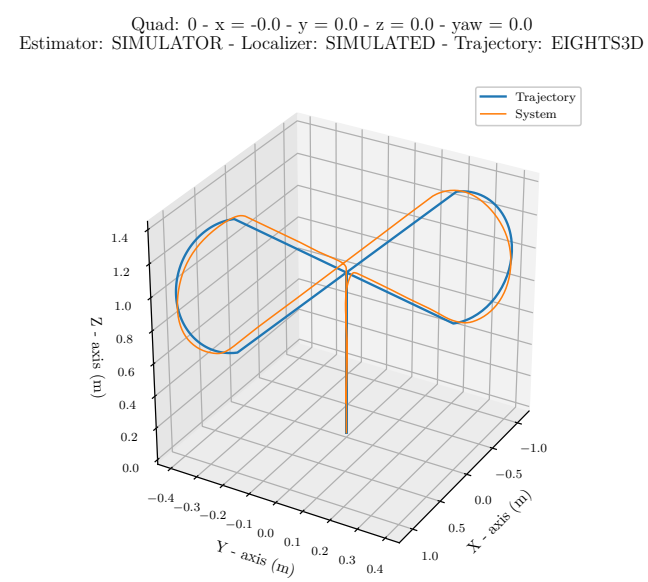

(c)

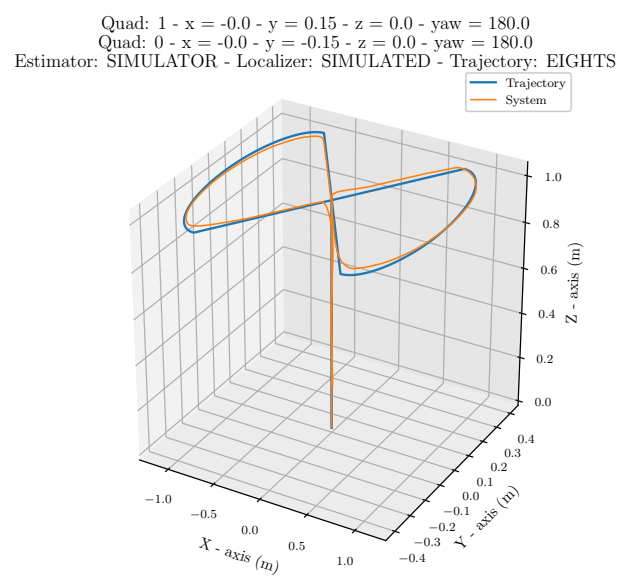

(b)

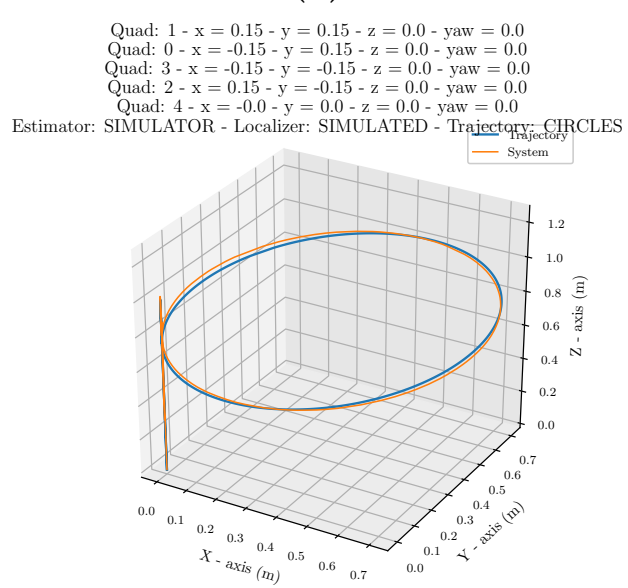

(d)

Figure 7.5: (a) 2D-Square: 3 quadcopters (b) 2D-Eight: 2 quadcopters (c) 3D-Eight: 1 quadcopter (d) 3D-Circles: 5 quadcopters

\subsubsection{Control Architecture and Strategy}

In Fig. 7.5, the output of a selection of the flights carried out in simulation is depicted. The plots are shown in 3D so its easier to get an overview of the behavior and the shape of the trajectories.

These examples show that the proposed control strategy provides system stability for different physical distributions, depicted in Fig. 7.2 , and number of quadcopters. The objective was not to focus on all the possible physical distribution, therefore, for $N>2$ cases only one 


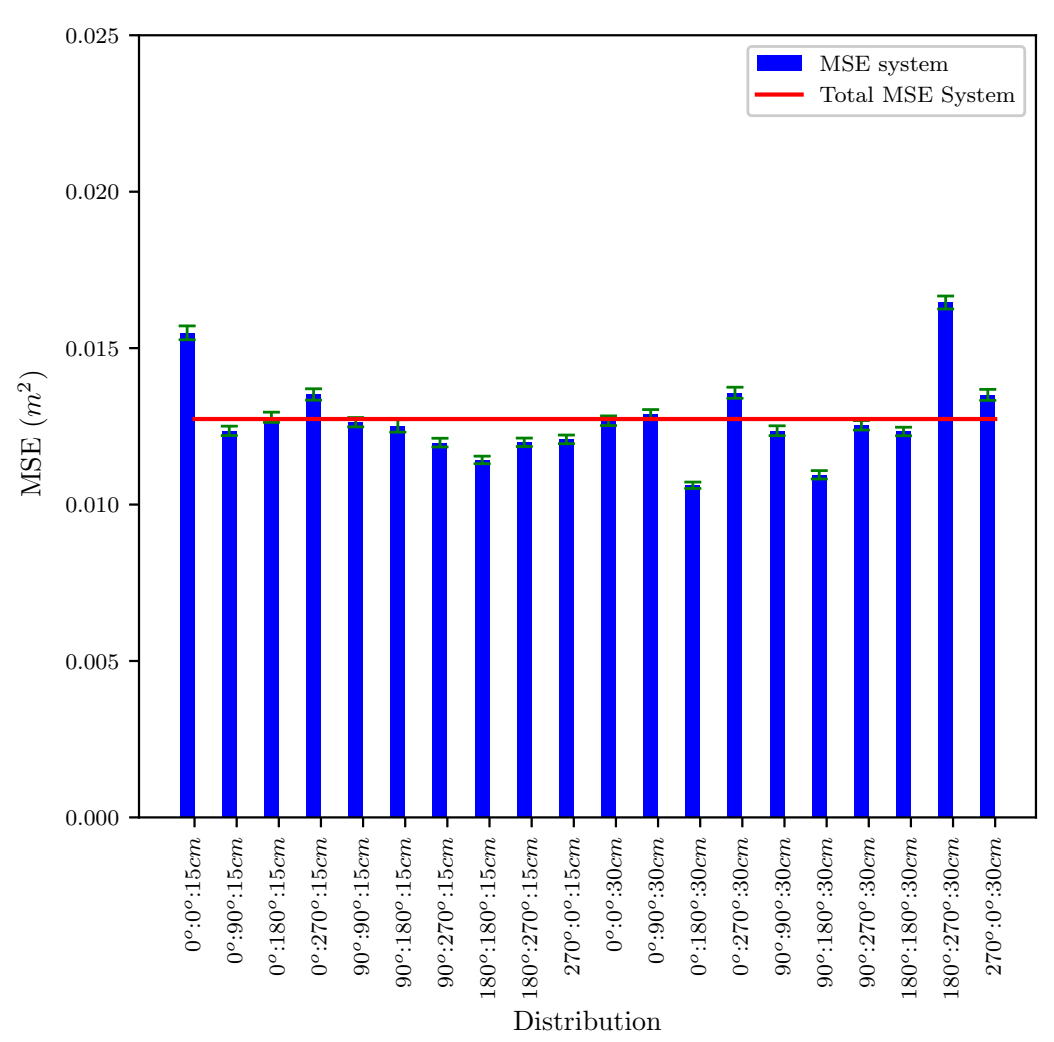

Figure 7.6: MSE distribution depending of the physical parameters of a 2-quadcopter system. In green, the variance of each MSE.

distribution was used in each case. As a future work, it is necessary to mathematically study the limitations along with the distributions in which system's stability is not ensured.

The last performed test was to apply all the trajectories to every possible 2-quadcopter distribution in order to validate that the performance of the controller would not vary with the distance $d_{01}$ between the quadcopters, or rotations around the z-axis $\vartheta_{0}$ and $\vartheta_{1}$. In Fig. 7.6 the MSE obtained for each physical distribution is plotted. This figure shows that these MSE values does not vary much from the mean MSE value. This fact serves as the validation in simulation for the proposed Control strategy and system architecture.

In Fig. 7.6, the MSE errors are greater in some configurations, around $\sim 0.015 \mathrm{~m}^{2}$ in the worst cases. These irregularities are explained 

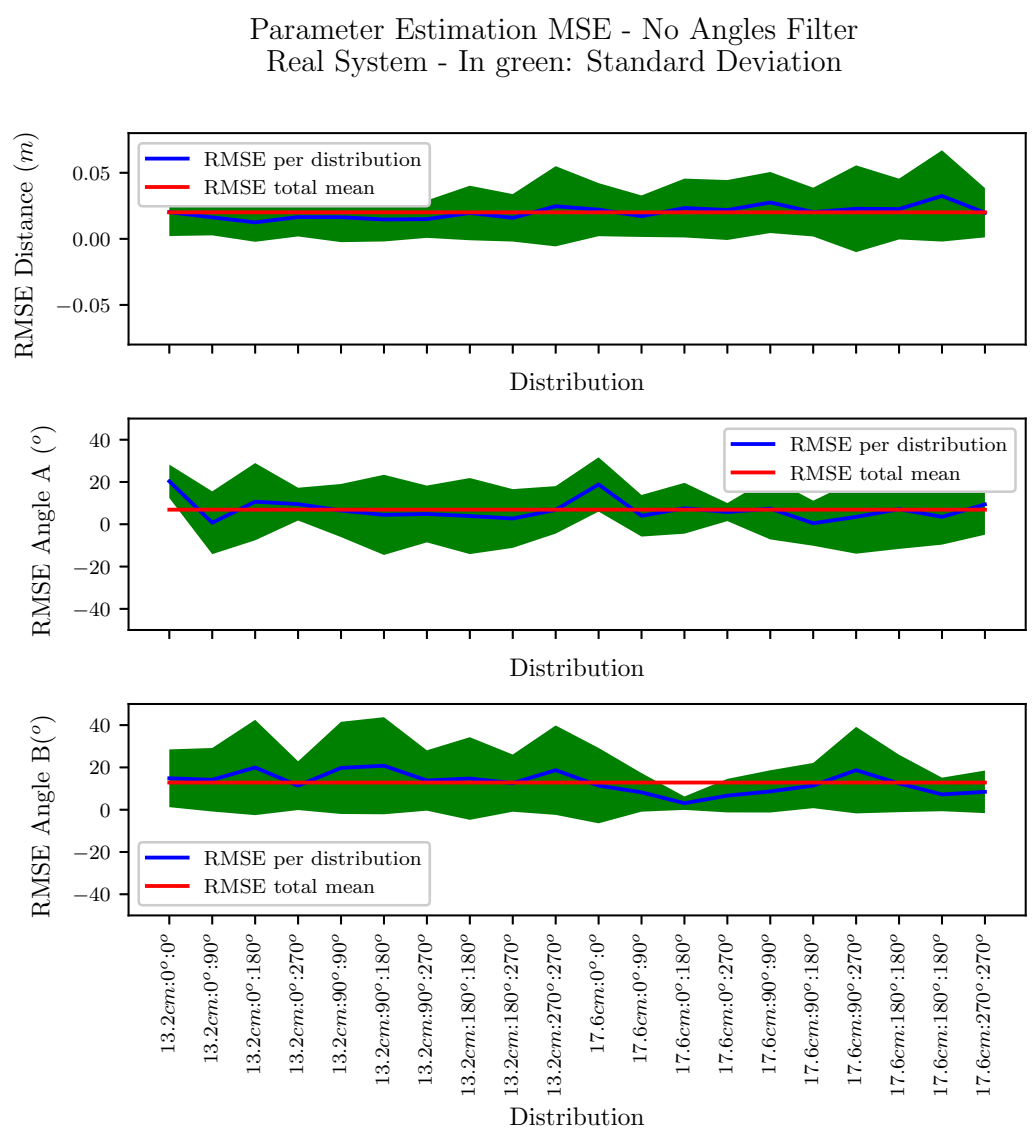

Figure 7.7: RMSE of the estimated physical parameters depending of the physical distribution. In green, the variance of each RMSE.

by the fact that the noise and bias introduced in the simulated system that adds certain stochastic behavior to the system. This can be observed in the values of the variances, greater in the cases that the MSE is further from the mean value.

\subsection{Experimental Results}

\subsubsection{Parameter Estimation Experiments}

In Fig. 7.7 the plot of the calculated RMSE values is shown. Logically, the obtained errors are slightly higher, but still inside reasonable limits ( $<0.015 \mathrm{~mm}$ and $<15$ degrees.).

The value of the error in the rotation around the $\mathrm{z}$-axis estimations 
Parameter Estimation MSE - Angles Filter

Real System - In green: Standard Deviation
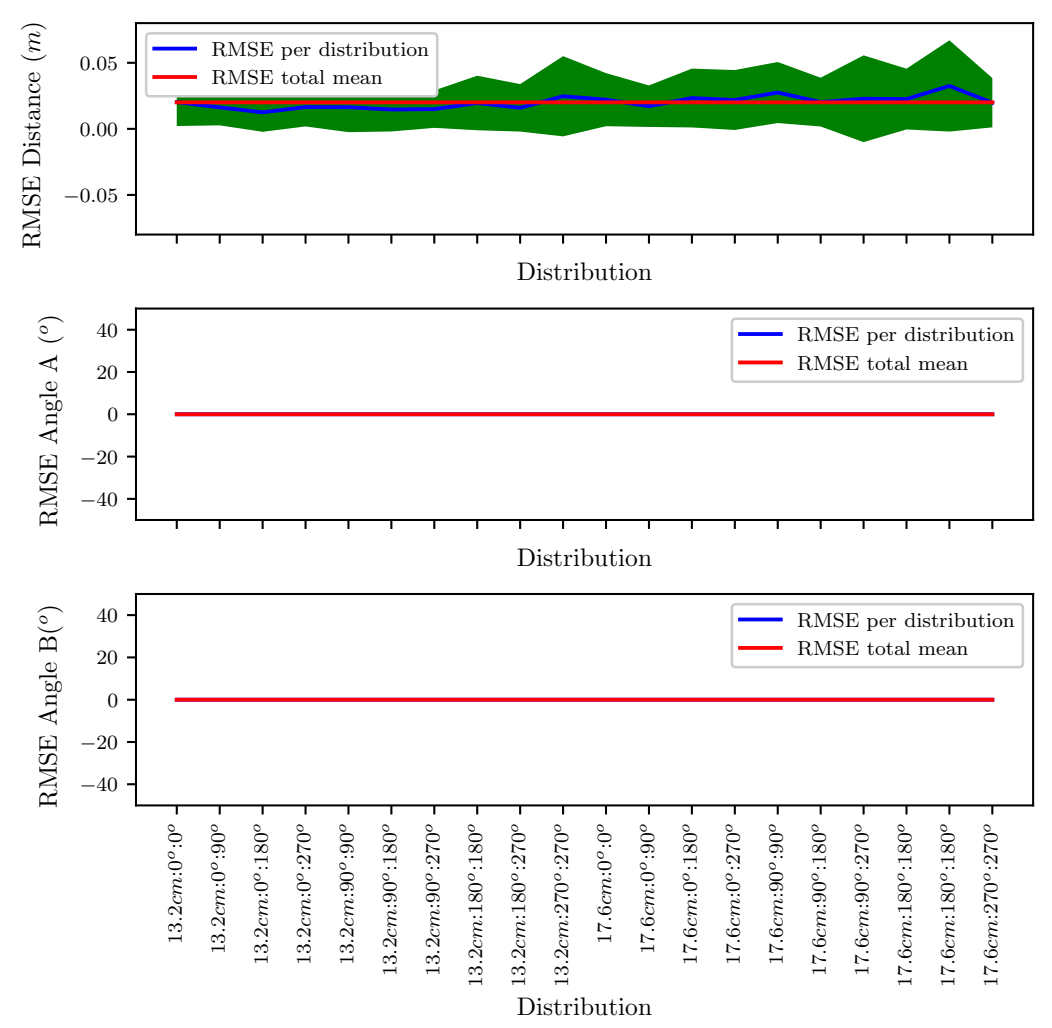

Figure 7.8: RMSE of the estimated physical parameters depending of the physical distribution. In green, the variance of each RMSE.

can be problematic. However, same rounding strategy used for the simulated case can be applied, obtaining then the RMSE values depicted in Fig. 7.8 .

In Fig. 7.8, it can be observed that the RMSE value decreases to zero, meaning that in every case the estimated angles were correctly classified. The parameter estimation method is validated for the rotation around the z-axis estimation.

\subsubsection{Control Architecture and Strategy}

\section{Flight Controller Experiments: MOtion CAPture System}

In Fig. 7.9 (a) the measured data for a single Crazyflie following the 2D-square trajectory is plotted. In Fig. 7.9(b), the output for the same 
2D-square trajectory is represented for the case of the 2 Crazyflies system. Comparing both plots gives an initial positive insight of the performance of the system as both followed trajectories are similar.

However, in the $x$ and $y$-axis, the system of Crazyflies shows a slower response. This may be due to two main reasons. First, the position and linear velocities controllers' gains has not been re-tuned as it has been left as a future work implementation. However, the inertia of the system is considerably different to the single Crazyflie case and logically the response varies.

The second reason is that the estimated PID gains for the attitude and angular velocities may not be optimal as the Reinforcement Based tuning was done using the simulator, which does not model all the possible imperfections (errors, biases, noise...) that can appear in the system.

The next step was to test the second trajectory: the 2D-eights. In Fig. 7.10, the path followed by the system of Crazyflies (b) is represented, in comparison with the output obtained by the single Crazyflie case (a).

The response of the system of 2 attached Crazyflies to the set-points determined by the 3D-circle trajectory is plotted in Fig. 7.11. 
Quad: $\mathrm{L}-\mathrm{x}=0.0-\mathrm{y}=0.0-\mathrm{z}=0.0-$ yaw $=0.0$ Estingtor: TRUE - Loclizer: MOCAP - Trajectory: SEQUENCE

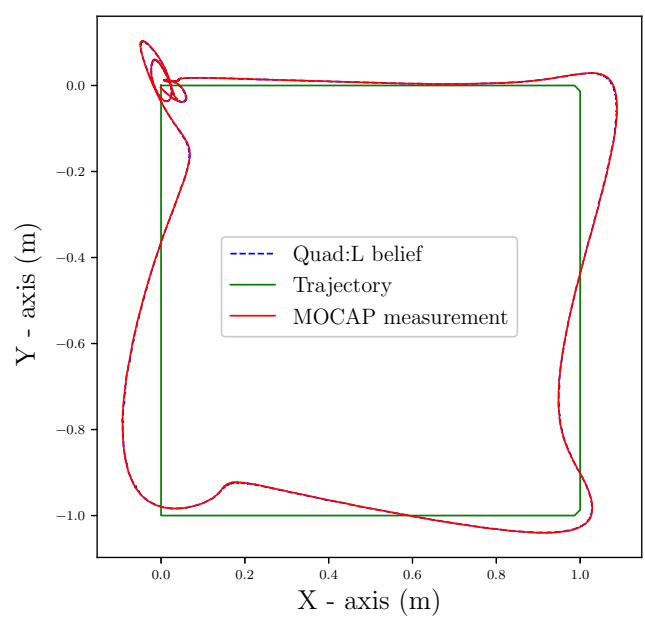

Quad: $\mathrm{L}-\mathrm{x}=0.0-\mathrm{y}=0.0-\mathrm{z}=0.0-$ yaw $=0.0$ Estimator: TRUE - Localizer: MOCAP - Trajectory: SEQUENCE
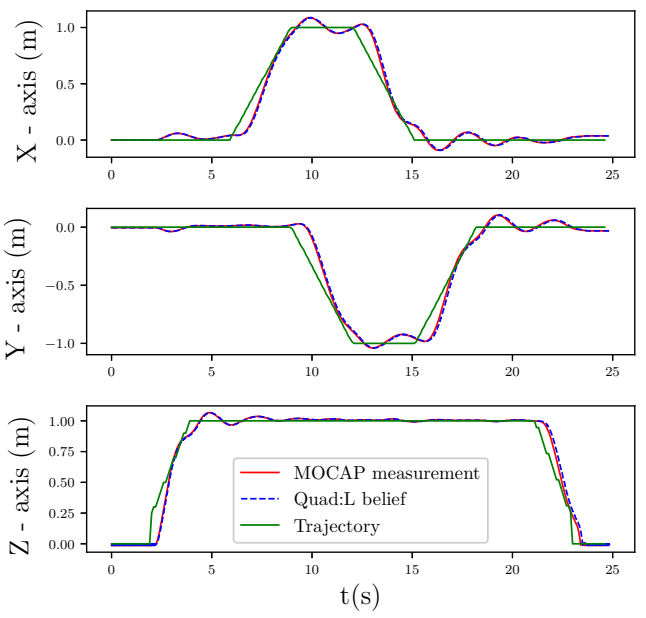

(a)
Quad: $\mathrm{s}-\mathrm{x}=-0.066-\mathrm{y}=0.0-\mathrm{z}=0.0-$ yaw $=0.0$ Estimator: TRUE - Localizer: MOCAPLEADER - Trajectory: SEQUENCE

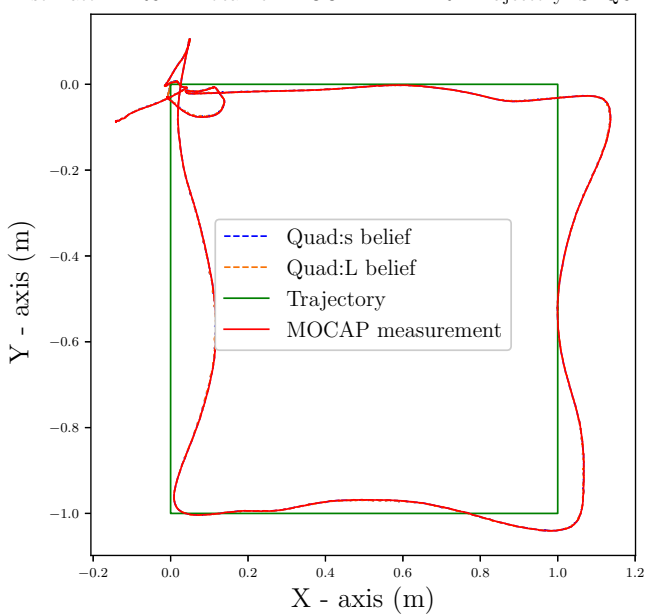

Quad: $\mathrm{s}-\mathrm{x}=-0.066-\mathrm{y}=0.0-\mathrm{z}=0.0-$ yaw $=0.0$ Quad: $\mathrm{L}-\mathrm{x}=0.066-\mathrm{y}=0.0-\mathrm{z}=0.0-$ yaw $=0.0$
Estimator: TRUE - Localizer: MOCAPLEADER - Trajectory: SEQUENCE
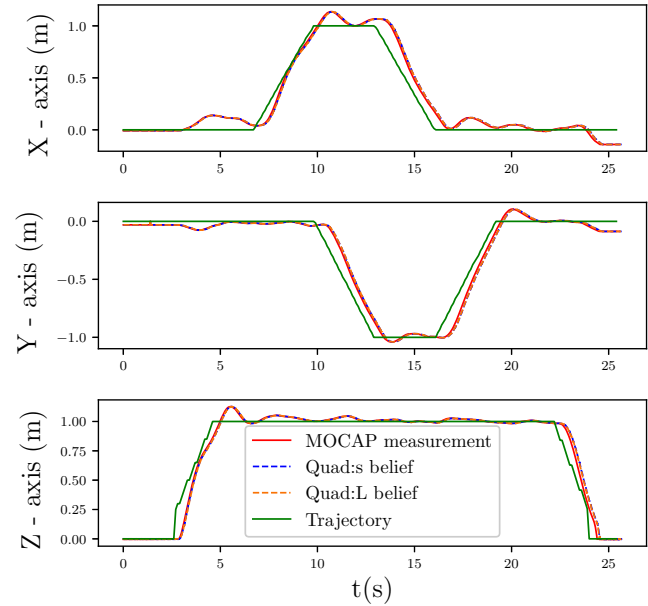

(b)

Figure 7.9: (a): Single Crazyflie's measured position using the MOCAP system and the original firmware. Sequence: 2D-square.(b) Dual Crazyflie System flight for the case of true physical parameters and the real time MOCAP measurements sent to the leader Crazyflie. Sequence: 2D-square. 

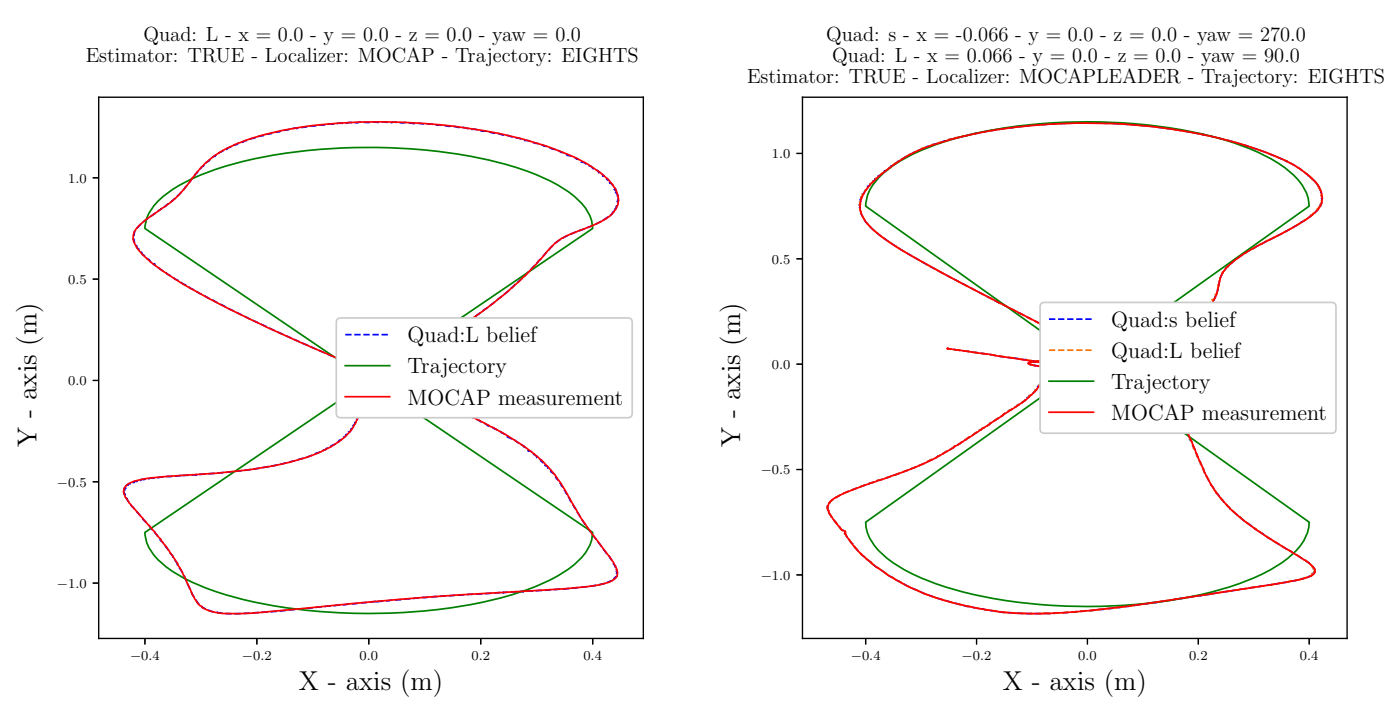

Quad: $\mathrm{L}-\mathrm{x}=0.0-\mathrm{y}=0.0-\mathrm{z}=0.0-$ yaw $=0.0$ Estimator: TRUE - Localizer: MOCAP - Trajectory: EIGHTS
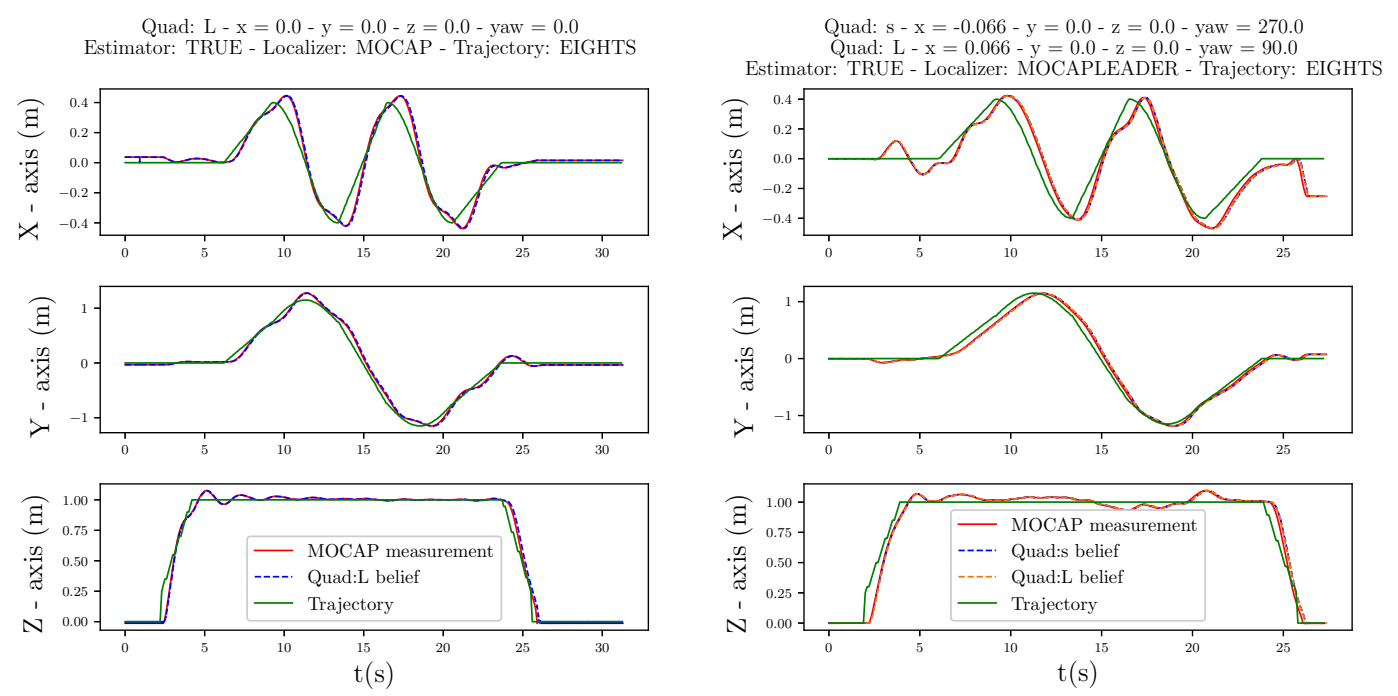

(a)

(b)

Figure 7.10: (a) Single Crazyflie's measured position using the MOCAP system and the original firmware. Sequence: 2D-eight. (b) Dual Crazyflie System flight for the case of true physical parameters and the real time MOCAP measurements sent to the leader Crazyflie. Sequence: 2D-eight. 


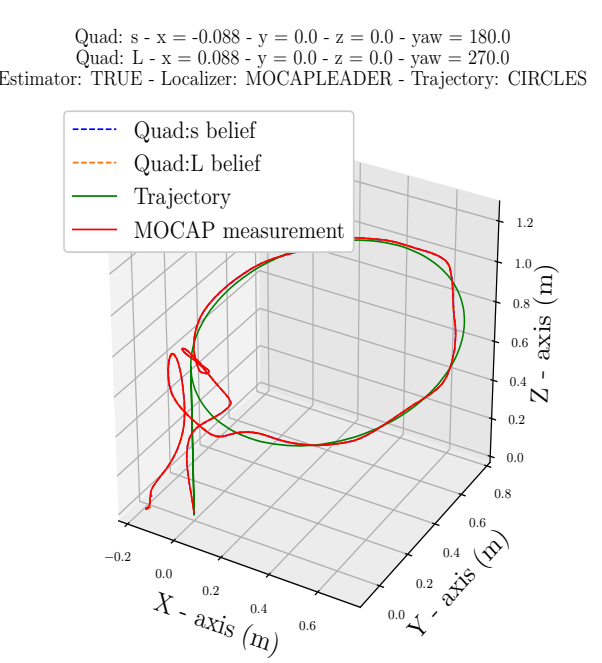

(a)

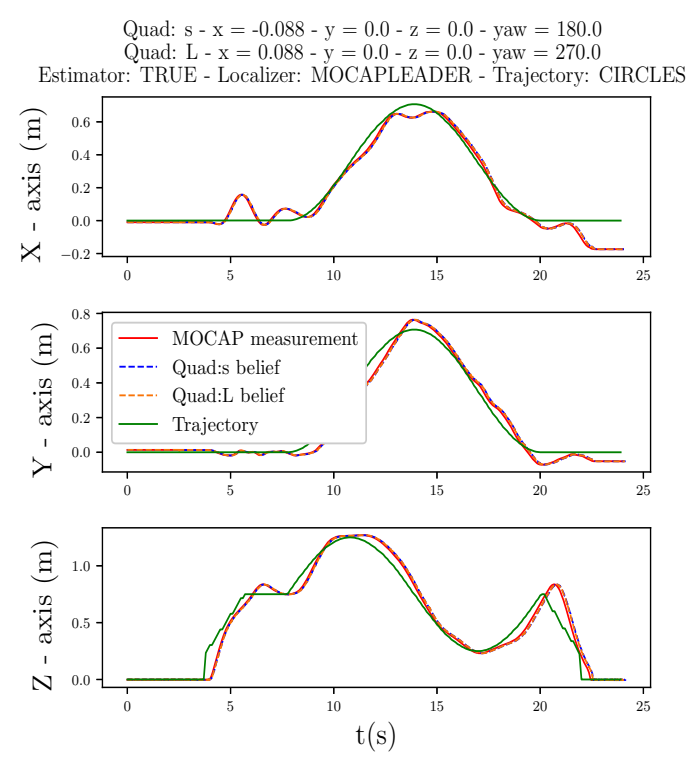

(b)

Figure 7.11: Dual Crazyflie System flight for the case of true physical parameters and the real time MOCAP measurements sent to leader Crazyflie. Sequence: 3D-circle.

In order to compare these errors with the one obtained in the single Crazyflie case, the total MSE of the 3 flights performed by the Crazyflie was calculated. These values are shown in Fig. 7.12. It can be observed how the MSE error for the dual-quadcopter case is low, with a maximum of $\sim 0.02 \mathrm{~m}^{2}$. Obtaining similar error values to the ones obtained for the single Crazyflie can be considered as another fulfilled validation bullet.

A better insight of the distribution of the MSE can be derived from dividing the MSE errors in the 3 axis. This way, it can be observed if any of the axis produces higher error values. In Fig. 7.13, the MSE errors are calculated for every axis and every distribution. The distribution of the error is almost invariant along the three axis, showing the z-axis lower values. However, as it was predicted before, the error values are higher along the $x$ and $y$-axis due to the changes in the inertia matrix and the constant values of the position and linear velocities PID gains. Observe from both Fig. 7.12 and Fig. 7.13 the MSE values are not affected by the value of the distance between the Crazyflies $d_{01}$ or the rotation around the z-axis of each of them $\vartheta_{0}^{o}$ and $\vartheta_{1}^{o}$, with the exception of the fourth distribution, with higher MSE. However, in this 
Total MSE comparison

True parameter values - MOCAP measurement sent to the Leader Single CF

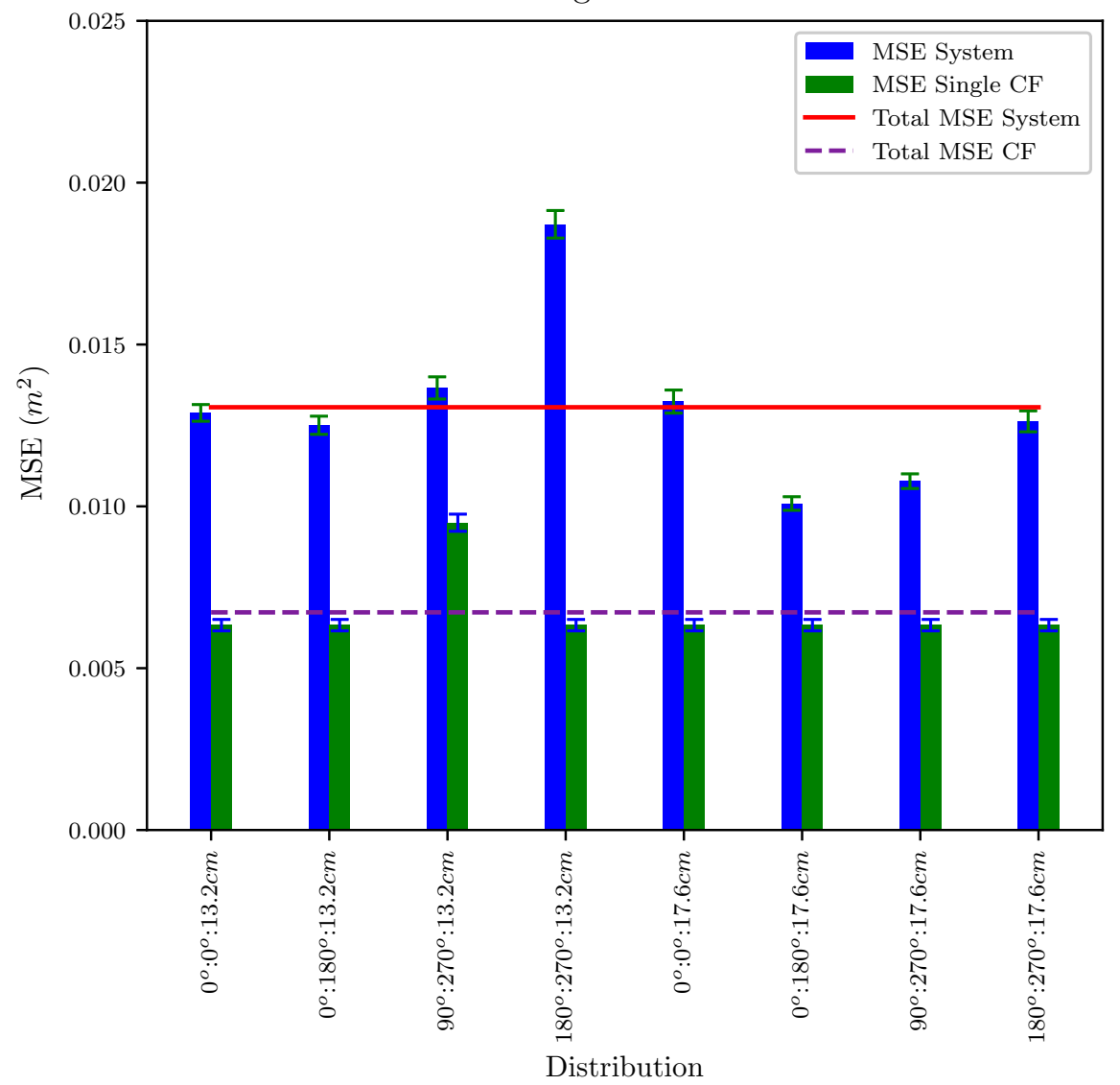

Figure 7.12: MSE comparison between the 2 attached Crazyflies and the single Crazyflie case. The single Crazyflie only performed 3 flights so only one MSE value is obtained that is repeated along the x-axis.

case, the variance is higher than the in the other cases, showing that the increased MSE value is caused by an isolated experiment in which the system performed worse.

These results serve as validation of the Power Distribution block equations (Eq. 5.5). The main objective of this block was to map the output of the angular velocities controller with the correspondent PWM values. This mapping allows the system's controller to be invariant to the possible values of $\vartheta_{i}^{o}$. 
Per axis MSE Error - True parameter values

- MOCAP measurement sent to Leader

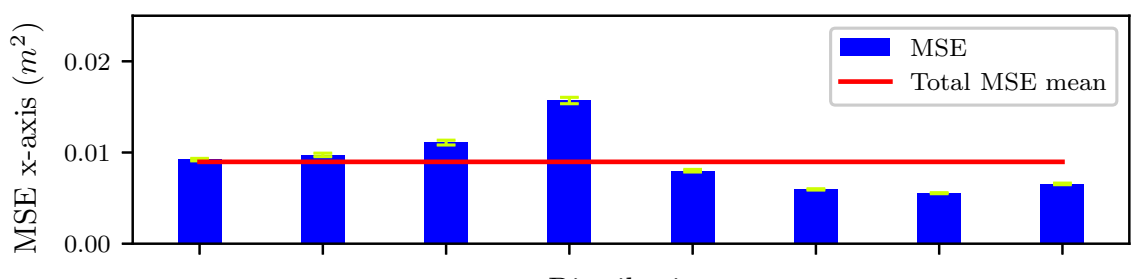

Distribution

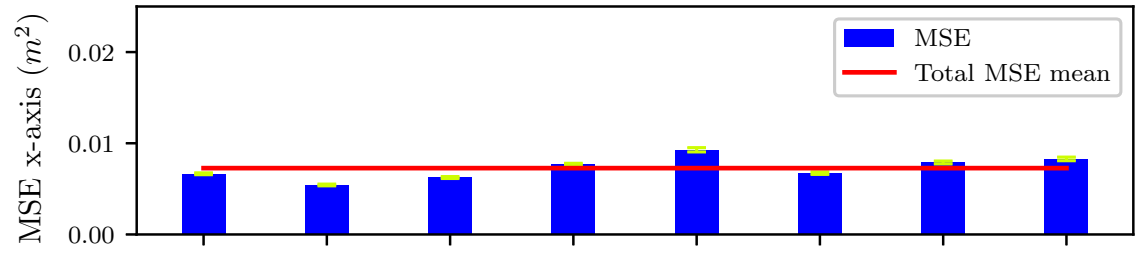

Distribution

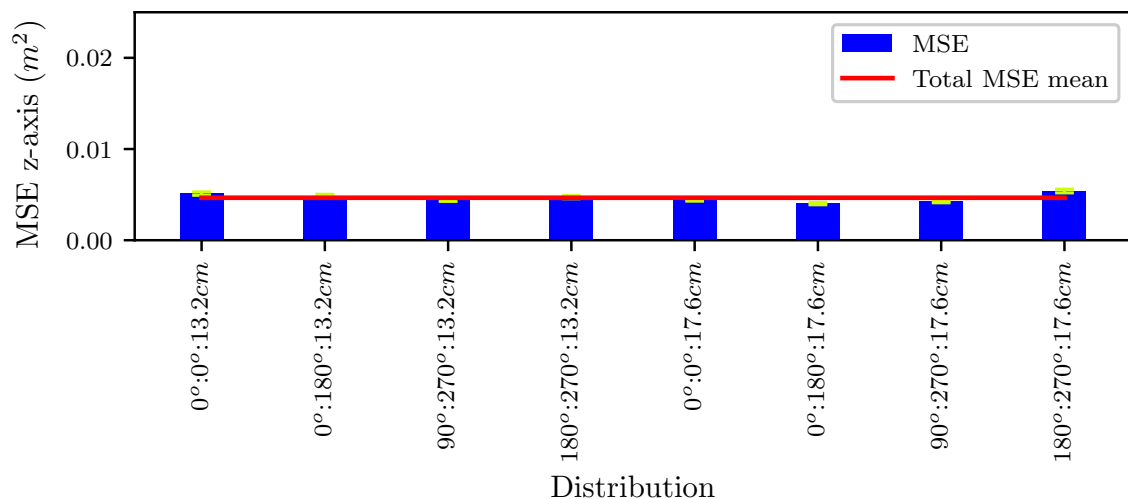

Figure 7.13: MSE values obtained in each axis from the data stored after 24 flights.

\section{Flight Controller Experiments: Loco Positioning System}

The LPS system showed a poor tracking performance during the experimentation step. The distance estimations to some of the anchors were noisy, and therefore, the position tracking was inaccurate and unstable. It can be observed in Fig. 7.14(a) that the position estimated by the LPS system in the single Crazyflie case, is significantly deviated from the one measured by the MOCAP system. This could be caused by different reasons, such as erroneous configuration, not accurate anchors' position measurements or even hardware malfunctioning.

However, several experiments were carried out to validate the sta- 


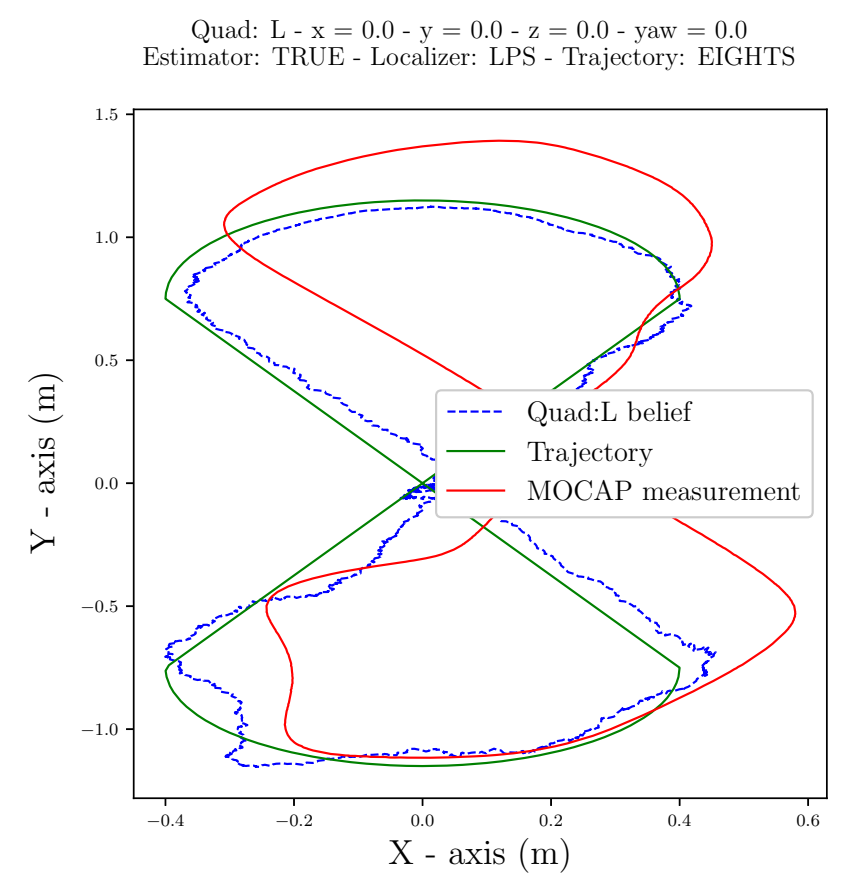

Figure 7.14: Single Crazyflie's measured $x, y, z$ positions using the MOCAP system as ground truth and LPS system for tracking, with the original firmware. Sequence: 2D-Eight.

bility of the system using the proposed global localization method with a different position tracker, the LPS. In this case, only one distribution was tested with all the trajectories. It can be observed in Fig. 7.15 that the stability is obtained even with very noisy and inaccurate position tracking.

The distribution used is $d_{01}=0.176 \mathrm{~m}$, with $\vartheta_{0}=90^{\circ}$ and $\vartheta_{1}=270^{\circ}$. After applying all the trajectories to this distribution, the MSE between the MOCAP measurements and the desired trajectory was calculated with a value of $0.2494035 \mathrm{~m}^{2}$ and a variance of $0.16707 \mathrm{~m}^{2}$

\section{Real-time Position Estimation Validation}

Finally, as the Kalman filter inside each Crazyflies runs independently and asynchronously, it was important to check the differences in the position belief in each Crazyflie. Big distances between both beliefs would mean a increase in the instability of the system that may lead to critical stability situations. 

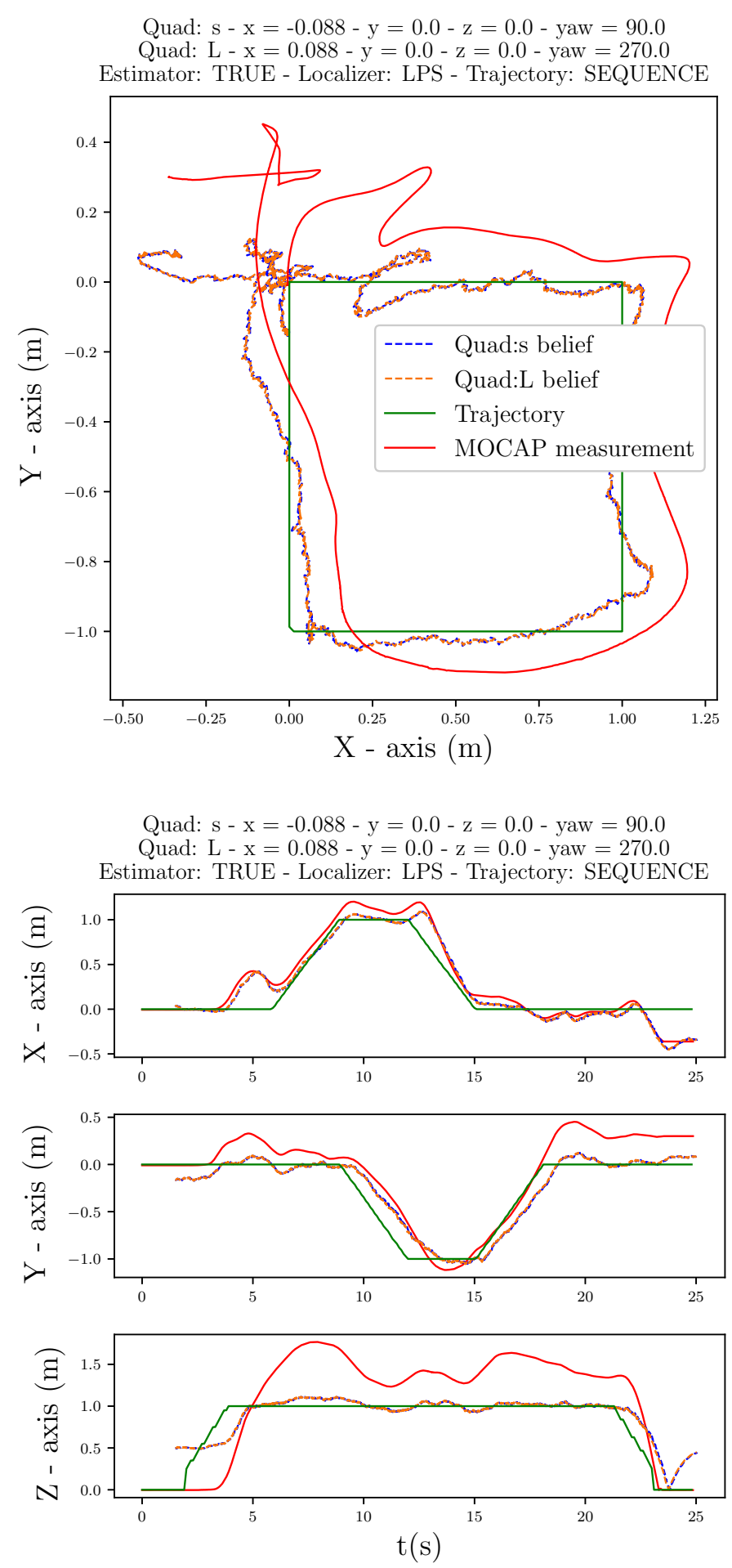

Figure 7.15: Single Crazyflie's measured $x, y, z$ positions using the MOCAP system as ground truth and LPS system for tracking, with the original firmware. Sequence: 2D-Eight. 
Total Belief differences - True parameter values

- MOCAP measurement sent to Leader
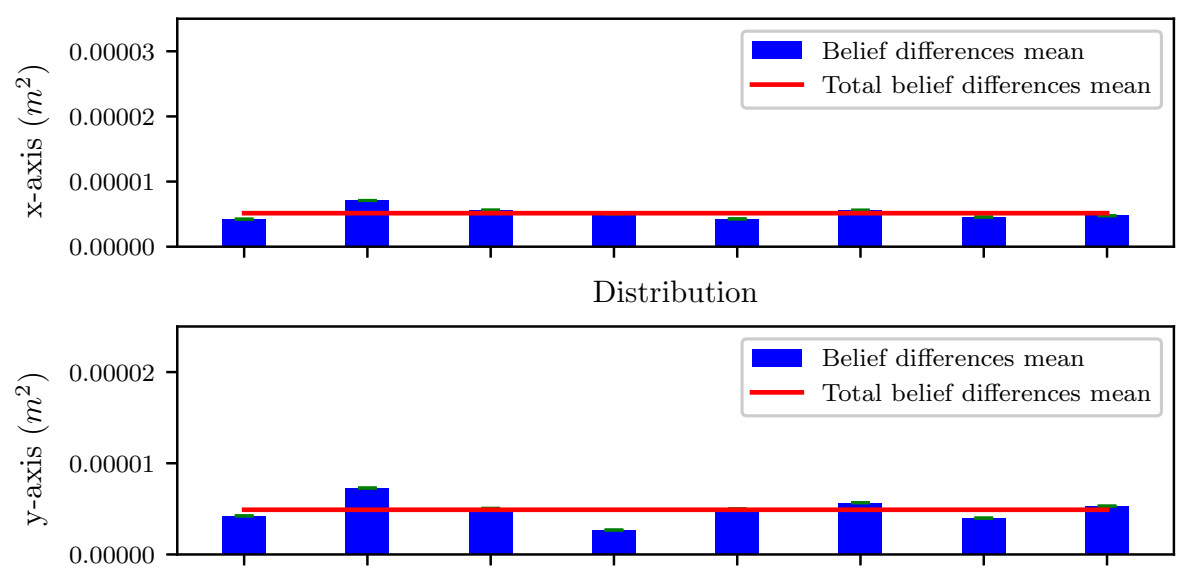

Distribution

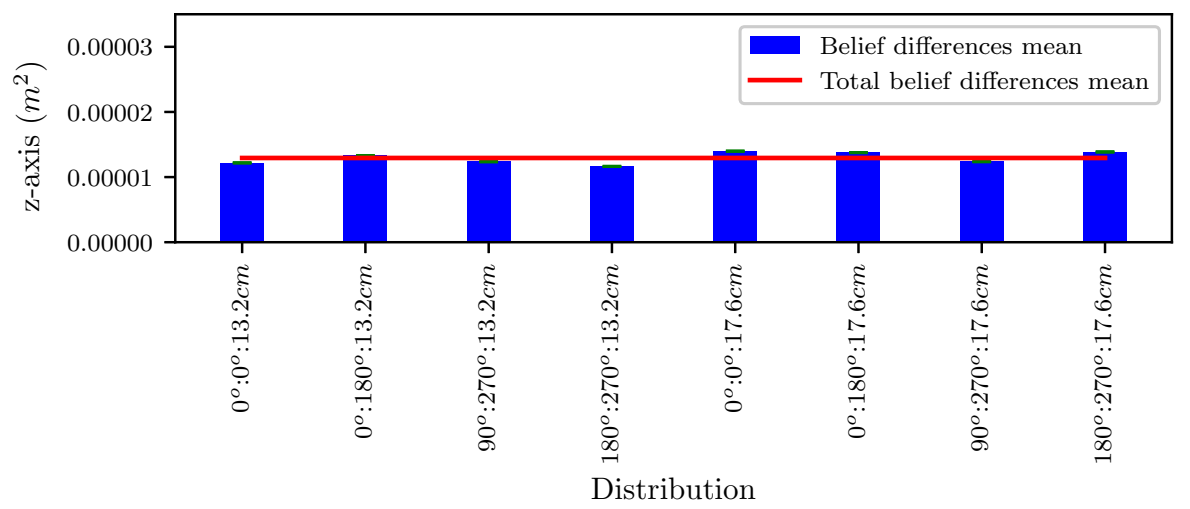

Figure 7.16: MSE values of the position belief difference inside each Crazyflie Kalman Filter.

The individual beliefs are plotted in every figure but their difference is too small to be observed. For this reason, the MSE of these differences has also been calculated, for each physical parameter distribution and each axis is plotted in Fig. 7.16. This figure shows that the values of this difference are very low, with maximums of around $10^{-5} \mathrm{~m}^{2}$. The differences has shown to be higher in the $\mathrm{z}$-axis, in some cases more than the double, this is justified by the fact that the accelerations are stronger in the $\mathrm{z}$-axis as the thrust is applied in that direction, which produces a faster drift in the $z$-axis belief. 


\subsubsection{Full Approach: Parameter Estimation and Con- trol Architecture}

Table 7.2 shows the estimated values obtained for each of the used distributions.

\begin{tabular}{|c|c|c|c|c|c|}
\hline$d_{01}$ & $\vartheta_{0}$ & $\vartheta_{1}$ & $d_{01}^{\prime}$ & $\vartheta_{0}^{\prime}$ & $\vartheta_{1}^{\prime}$ \\
\hline $176 \mathrm{~mm}$ & $0^{\circ}$ & $0^{\circ}$ & $178.48 \mathrm{~mm}$ & $0^{\circ}$ & $0^{\circ}$ \\
$176 \mathrm{~mm}$ & $180^{\circ}$ & $0^{\circ}$ & $202.9 \mathrm{~mm}$ & $180^{\circ}$ & $0^{\circ}$ \\
$176 \mathrm{~mm}$ & $180^{\circ}$ & $270^{\circ}$ & $176 \mathrm{~mm}$ & $180^{\circ}$ & $270^{\circ}$ \\
$176 \mathrm{~mm}$ & $270^{\circ}$ & $90^{\circ}$ & $198.02 \mathrm{~mm}$ & $270^{\circ}$ & $90^{\circ}$ \\
\hline $132 \mathrm{~mm}$ & $0^{\circ}$ & $0^{\circ}$ & $139.836 \mathrm{~mm}$ & $0^{\circ}$ & $0^{\circ}$ \\
$132 \mathrm{~mm}$ & $180^{\circ}$ & $0^{\circ}$ & $122.68 \mathrm{~mm}$ & $180^{\circ}$ & $0^{\circ}$ \\
$132 \mathrm{~mm}$ & $180^{\circ}$ & $270^{\circ}$ & 133.692 & $180^{\circ}$ & $270^{\circ}$ \\
$132 \mathrm{~mm}$ & $270^{\circ}$ & $90^{\circ}$ & $152.4 \mathrm{~mm}$ & $270^{\circ}$ & $90^{\circ}$ \\
\hline
\end{tabular}

Table 7.2: Combination of values of $\vartheta_{0}, \vartheta_{1}$ and $d_{01}$ that will be used in the experiments.

The trajectory followed for one of the tested cases is shown in Fig 7.17. In the top figure, the $3 \mathrm{D}$ path followed by the system is plotted. This figure is plotted to show what happens when the distance estimation is not accurate. The path followed in the $\mathrm{x}$-axis shows a greater overshooting than the behavior obtained in the $y$ and $z$-axis. Besides, this overshooting is higher than in any other case depicted above. The Crazyflies were situated along the y-axis in this case, relative to the MOCAP coordinate system. Therefore, the distance of the Crazyflies to the x-axis is zero, and the PID gains for the roll and $w_{x}$ velocities are unchanged. On the contrary, the pitch and $w_{y}$ PID gains were mapped using the estimated distance between the Crazyflies. As a consequence, if the estimation of this distance is not accurate, the PID gains would be further from the optimal values, and the performance along this axis would decrease. In this case, the overshooting was higher. The system still shows full stability either ways. 
Quad: s - x $=-0.0962017797793-\mathrm{y}=0.0-\mathrm{z}=0.0-$ yaw $=-180.0$

Quad: L - $\mathrm{x}=0.0962017797793-\mathrm{y}=0.0-\mathrm{z}=0.0-$ yaw $=-90.0$

Estimator: ESTIMATED - Localizer: MOCAPLEADER - Trajectory: EIGHTS

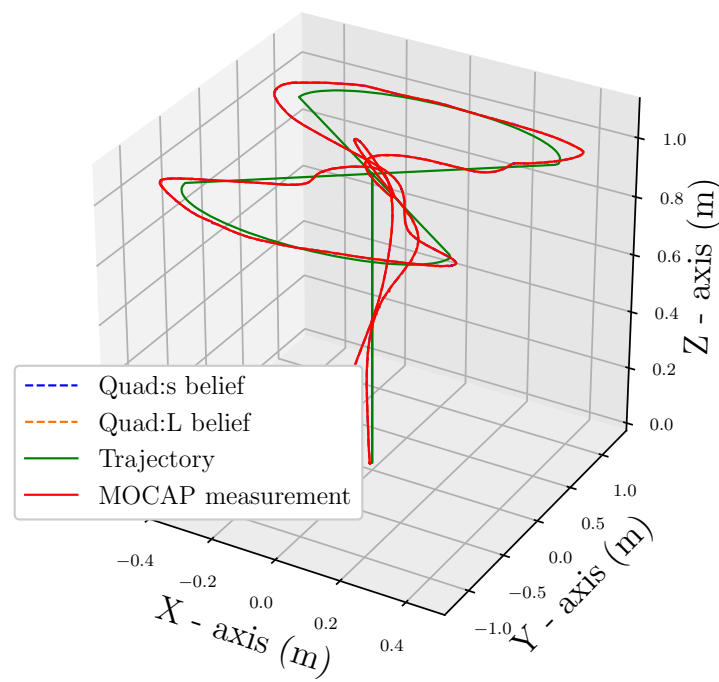

Quad: $\mathrm{s}-\mathrm{x}=-0.0962017797793-\mathrm{y}=0.0-\mathrm{z}=0.0-$ yaw $=-180.0$

Quad: $\mathrm{L}-\mathrm{x}=0.0962017797793-\mathrm{y}=0.0-\mathrm{z}=0.0-\mathrm{yaw}=-90.0$

Estimator: ESTIMATED - Localizer: MOCAPLEADER - Trajectory: EIGHTS
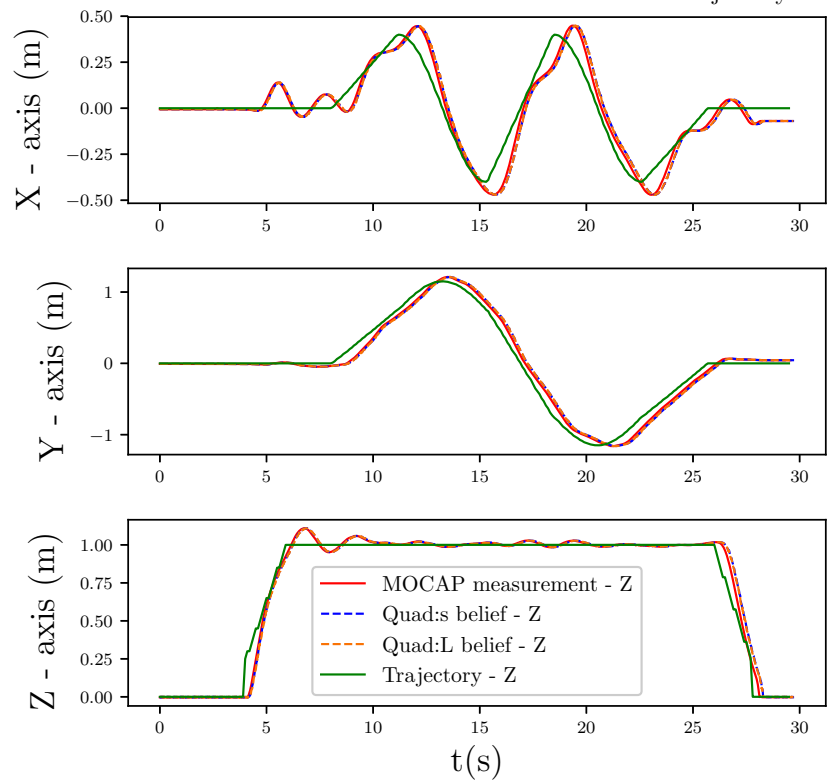

Figure 7.17: Dual Crazyflie System flight for the case of estimated physical parameters and the real time MOCAP measurements sent to the leader $L$. Top: 3D view. Bottom: Time evolution per axis. Sequence: 2D-eights. 
Total MSE comparison :

True parameter values - MOCAP measurement sent to Leader Estimated parameter values - MOCAP measurement sent to Leader

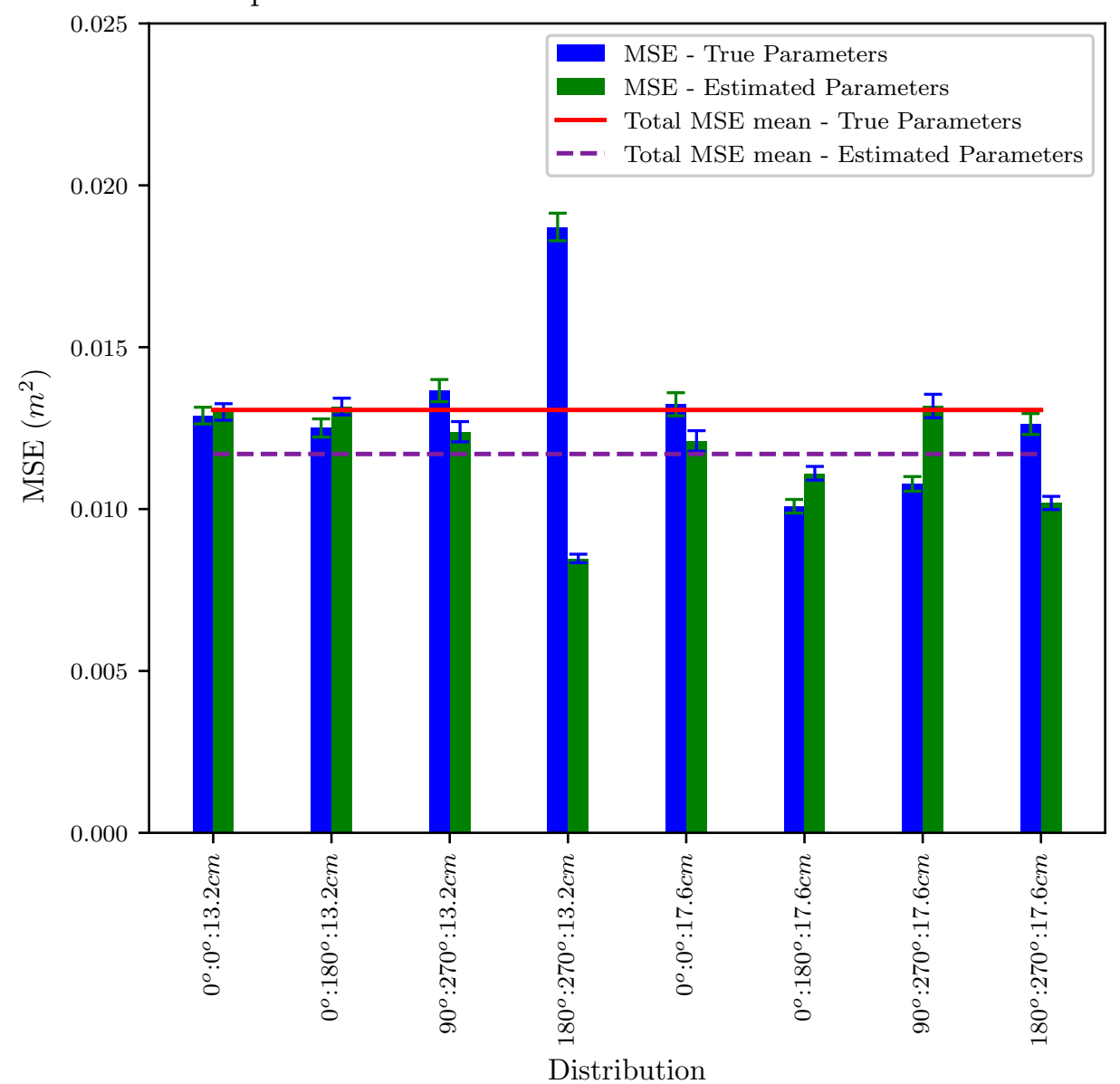

Figure 7.18: MSE comparison between the 2 attached Crazyflies and the single Crazyflie case. The single Crazyflie only performed 3 flights so only one MSE value is obtained that is repeated along the $\mathrm{x}$-axis.

The comparison of the obtained errors with the last case scenario is shown in Fig. 7.18. It can be observed that the total error remains in the same units with a small improvement $\left(<0.002 \mathrm{~m}^{2}\right)$. However, this improvement can not be used to take any conclusion as it was already explained due to the different external variables that may affect the total error. The important conclusion is that the performance does not vary significantly when the system uses the estimated parameters compared to the case in which the true parameters are sent. 


\section{Chapter 8}

\section{Conclusions and Future Work}

\subsection{Conclusion}

In the current thesis, a method to flight a system of attached quadcopters, with unknown physical connection parameters by integrating an adaptive controller structure with a novel method to estimate these parameters has been proposed.

First, a method to estimate the physical connection parameters of rigidly attached quadcopters was implemented, solely relying on the information from the quadcopters' Inertial Measurement Units (IMU). This estimation was achieved via simple and short online experiments. A variance-based approach to filter the IMU measurements to get useful information was proposed and implemented. Furthermore, a novel strategy to use the filtered measurements to extract complex geometrical relationships between the attached quadcopters has been developed.

The possible combinations of the number of quadcopters and their positions and orientations of the system of rigidly attached quadcopters is infinite, and the design of the control strategy was one of the main challenges. In the current thesis, an adaptive control structure was proposed. Besides, a reinforcement learning algorithm, based in Deep Q-Learning, to tune the PID gains of the controller has been implemented.

One challenge of the system was the global positioning, as generally, only individual quadcopters' position can be measured. This issue was solved by measuring only the position of one of the quadcopters that would estimate the position of the system, using the con- 
straints derived from the characteristics of the physical connection. This estimated position was then sent to the rest of the quadcopters in the system in real time. The proposed method simplifies the problem while reduces the economic costs and power consumption as only one of the quadcopters will be in charge of measuring the position of the system. This method allows to use both on-board sensors, as in [41], and other positioning methods based in external measurements such as UWB, Motion Captures systems or GPS.

The proposed approach to estimate the physical connection parameters of rigidly attached quadcopters has been validated both in simulation and in the real system. Besides, the proposed algorithm has shown enough accuracy to allow to directly fly the system using the estimated parameters. In the experimentation, it has been shown that is possible to obtain useful data from IMUs that can be efficiently used to estimate these parameters.

The proposed controller structure was validated both in simulation and the real system using a varied set of distributions, proving that the implemented control law ensures a stable flight independently of the combination of the number of quadcopters, their positions or orientations.

Both the simulation and experimentation with the real system showed that only the position of one quadcopter needs to be measured, as this quadcopter can estimate the position of the system due to the geometrical constraints of the system and the latency in this communications has been observed to be negligible.

Finally, the entire process has been validated in the experimentation with several distributions of the real system.

\subsection{Future Work}

The future work can be divided in six sections: hardware, simulator, parameter estimation, control, reinforcement learning and system positioning.

\section{Hardware}

The designed physical attachment could be considerably improved, first, reducing its weight using very light materials like carbon fiber. Besides, the attaching-detaching process is very tedious, it is necessary 
to design a smarter why to perform this process that could even allow its automatization.

\section{Parameter Estimation}

First, the data obtained in the process is very repeatable, or patternbased, and therefore, it is possible that a machine learning approach to analyze this data could improve considerably the accuracy and the speed. This might allow the system be self-assembled in mid-air. Then, the first research path would be to analyze and study this data deeper. Besides, if different patterns could be obtained from the data, it would also mean that significant estimation errors could be detected. Due to isolated communication lost, in some cases, the estimation process failed: not all the data was received and stored or the set-points sent to one of the quadcopters to achieve the dynamical oscillations were not received or were received late. This issues lead to erroneous estimations in some cases. The idea then would be, to detect the patterns that allows the system to predict when the estimation has been erroneous, and automatically repeat the estimation process.

\section{Control Architecture}

To conclude, regarding to the control architecture, the first step would be to studying the model of the system and researching in novel control methods. It would be interesting to implement a control architecture in which the parameter tunning could be perform online or could be directly derived from the dynamical model equations. Some examples of these kind of architectures were introduced in Section 1.3 . Besides, the dynamical model should be studied further to mathematically extract the limitations of the system, finding the combination of number of quadcopters and their positions and orientations that would not ensure stability.

\section{Reinforcement Learning}

The most interesting future research work related with the thesis is the implementation of an online reinforcement learning algorithm. This algorithm will have 2 main objectives: improve the estimated parameters of the proposed controller, and improve the estimation of the 
physical parameters. Both improvements would lead to a significant increase in the final performance of the system.

\section{Simulator}

In order to improve the control architecture, or test different approaches, it would be important to improve the simulator, adding a more accurate model of the IMUs in the selected quadcopters, and an improve mapping between the motors applied voltage and the obtained thrust.

\section{System Positioning}

Moving forward to the system positioning approach, it would be interesting to experiment with different positioning sensors or modules, such as camera-based estimators, as the ones used in [41]: This way, it could be proven that their approach could be implemented but using only one sensor, instead of having one in each quadcopter. Besides, the different methods will have different characteristics, such as processing rates or precision, that need to be tested. Then, another future task would be to determine the limitations of the proposed positioning studying the evolution of the latency as the number of attached quadcopters increases, and how it affects the stability of the system. 


\section{Bibliography}

[1] A. K. Akametalu, J. F. Fisac, J. H. Gillula, S. Kaynama, M. N. Zeilinger, and C. J. Tomlin. Reachability-based safe learning with gaussian processes. In 53rd IEEE Conference on Decision and Control, pages 1424-1431, Dec 2014.

[2] J. Angeles. Time Response of First- and Second-order Dynamical Systems. Mechanical Engineering Series. Springer.

[3] Kai Arulkumaran, Marc Peter Deisenroth, Miles Brundage, and Anil Anthony Bharath. A brief survey of deep reinforcement learning. CoRR, abs/1708.05866, 2017.

[4] F. Berkenkamp, A. P. Schoellig, and A. Krause. Safe Controller Optimization for Quadrotors with Gaussian Processes. ArXiv eprints, September 2015.

[5] Bitcraze. cflib: Crazyflie python library.

https://github.com/bitcraze/crazyflie-lib-python.

[6] Bitcraze. Crazyflie 2.0.

https://www.bitcraze.io/crazyflie-2/.

[7] Bitcraze. Crazyflie 2.0 firmware.

https://github.com/bitcraze/crazyflie-firmware.

[8] Bitcraze. Crazyradio. https://www.bitcraze.io/crazyradio/.

[9] Bitcraze. Loco positioning system.

https://www.bitcraze.io/loco-pos-system/.

[10] Bitcraze. Wiki page.

https://wiki.bitcraze.io/. 
[11] R.L. Burden and J.D. Faires. Numerical Analysis. Number v. 1 in Numerical Analysis. Brooks/Cole, 2001.

[12] Zheng Chen, Tae I. Um, and Hilary Bart-Smith. Bio-inspired robotic manta ray powered by ionic polymer-metal composite artificial muscles. International Journal of Smart and Nano Materials, 3(4):296-308, 2012.

[13] Bing Cheng and D. M. Titterington. Neural networks: A review from a statistical perspective. Statist. Sci., 9(1):2-30, 021994.

[14] decaWave. Decawave's DW1000 Ultra Wideband (UWB) transceiver IC. https://www.decawave.com/products/dwm1000-module.

[15] MP. Deisenroth and CE. Rasmussen. Pilco: A model-based and data-efficient approach to policy search. In Proceedings of the 28th International Conference on Machine Learning, ICML 2011, pages 465-472. Omnipress, 2011.

[16] Ananda G. K. Deters, R. W. and M. S. Selig. Reynolds number effects on the performance of small-scale propellers. In 32nd AIAA Applied AIAA Aviation, editor, Aerodynamics Conference, Atlanta, GA, June 2014, pp. 2014-2151. 2014.

[17] Boston Dynamics. Spotmini robot.

https://www.bostondynamics.com/spot-mini.

[18] Mh Elec. Mh-vtol2.

http:/ / www.mh-elec.com/photo-x.php?id=228.

[19] Philip R. Evans. Rotations and rotation matrices. Acta Crystallographica Section D, 57(10):1355-1359, Oct 2001.

[20] Martin A Fischler and Robert C Bolles. Random sample consensus: a paradigm for model fitting with applications to image analysis and automated cartography. In Readings in computer vision, pages 726-740. Elsevier, 1987.

[21] Julian Förster. System identification of the crazyflie 2.0 nano quadrocopter. Master's thesis, ETH, Swiss Federal Institute of Technology Zurich, Electrical Engineering, 2015. 
[22] Douglas W. Gage. Ugv history 101: A brief history of unmanned ground vehicle (ugv) development efforts. Unmanned Systems, 13:9-32, 1995.

[23] M. Gassner, T. Cieslewski, and D. Scaramuzza. Dynamic collaboration without communication: Vision-based cable-suspended load transport with two quadrotors. In 2017 IEEE International Conference on Robotics and Automation (ICRA), pages 5196-5202, May 2017.

[24] J. H. Gillula and C. J. Tomlin. Guaranteed safe online learning via reachability: tracking a ground target using a quadrotor. In 2012 IEEE International Conference on Robotics and Automation, pages 2723-2730, May 2012.

[25] GPS.gov. Gps accuracy.

[26] Marcus Greiff. Modelling and control of the crazyflie quadrotor for aggressive and autonomous flight by optical flow driven state estimation. Master's thesis, LUP, Lund University, Automatic Control, 2017.

[27] S. Gupte, Paul Infant Teenu Mohandas, and J. M. Conrad. A survey of quadrotor unmanned aerial vehicles. In 2012 Proceedings of IEEE Southeastcon, pages 1-6, March 2012.

[28] Martin T Hagan, Howard B Demuth, Mark H Beale, et al. Neural network design, volume 20. Pws Pub. Boston, 1996.

[29] HiBot. Acm-r5h: the amphibious snake-like robot. https://www.hibot.co.jp/ecommerce/prod-detail/14.

[30] Shun ichi Amari. Backpropagation and stochastic gradient descent method. Neurocomputing, 5(4):185 - 196, 1993.

[31] InvenSense. MPU 9250 Product Specification Revision 1.0. Rev. 1.

[32] Leslie Pack Kaelbling, Michael L. Littman, and Andrew W. Moore. Reinforcement learning: A survey. CoRR, cs.AI/9605103, 1996.

[33] Leslie Pack Kaelbling, Michael L. Littman, and Andrew W. Moore. Reinforcement learning: A survey. J. Artif. Int. Res., 4(1):237-285, May 1996. 
[34] Rudolf Kalman. A new approach to linear filtering and prediction problems. In Transactions of the ASME - Journal of basic Engineering, volume 82, pages 35-45, 011960.

[35] Juhyeok Kim, Hai-Nguyen Nguyen, and Dongjun Lee. Preliminary control design on spherically-connected multiple-quadrotor manipulator system. In 2015 12th International Conference on Ubiquitous Robots and Ambient Intelligence (URAI), pages 206-207, Oct 2015.

[36] M. Kok, J. D. Hol, and T. B. Schön. Using Inertial Sensors for Position and Orientation Estimation. ArXiv e-prints, April 2017.

[37] Benoit Landry. Planning and control for quadrotor flight through cluttered environments. 2014.

[38] H. Lee, H. Kim, and H. J. Kim. Planning and control for collisionfree cooperative aerial transportation. IEEE Transactions on $\mathrm{Au}$ tomation Science and Engineering, 15(1):189-201, Jan 2018.

[39] Ji Hong Li and Pan Mook Lee. A neural network adaptive controller design for free-pitch-angle diving behavior of an autonomous underwater vehicle. Robotics and Autonomous Systems, 52(2):132 - 147, 2005.

[40] Zhixiang Liu, Youmin Zhang, Xiang Yu, and Chi Yuan. Unmanned surface vehicles: An overview of developments and challenges. In Annual Reviews in Control, volume 41, 052016.

[41] G. Loianno and V. Kumar. Cooperative transportation using small quadrotors using monocular vision and inertial sensing. IEEE Robotics and Automation Letters, 3(2):680-687, April 2018.

[42] David M. Lovinger. Communication networks in the brain: Neurons, receptors, neurotransmitters, and alcohol. In National Institute on Alcohol Abuse and Alcoholism, volume 31, pages 196-214, 2008.

[43] LSTS. Light autonomous underwater vehicle lauv. https://lsts.fe.up.pt/vehicles/lauv. 
[44] X. Lu, S. Chen, C. Wu, and M. Li. The pulse width modulation and its use in induction motor speed control. In 2011 Fourth International Symposium on Computational Intelligence and Design, volume 2, pages 195-198, Oct 2011.

[45] C. Luis and J. Le Ny. Design of a trajectory tracking controller for a nanoquadcopter. Master's thesis, École Polytechnique de Montréal, Automatic Control, 2016.

[46] Teppo Luukkonen. Modelling and control of quadcopter. Independent research project in applied mathematics, Espoo, 22, 2011.

[47] R. Mahony, T. Hamel, and J. M. Pflimlin. Nonlinear complementary filters on the special orthogonal group. IEEE Transactions on Automatic Control, 53(5):1203-1218, June 2008.

[48] Teledyne Marine. Gavia auv.

http:/ / www.teledynemarine.com/gavia-auv.

[49] Jörg Sander Martin Ester, Hans-Peter Kriegel and Xiaowei Xu. A density based algorithm for discovering clusters in large spatial databases with noise. In AAAI KDD-96. AAAI, 1996.

[50] Daniel Mellinger, Michael Shomin, Nathan Michael, and Vijay Kumar. Cooperative grasping and transport using multiple quadrotors. In Distributed Autonomous Robotic Systems: The 10th International Symposium, pages 545-558, Berlin, Heidelberg, 2013. Springer Berlin Heidelberg.

[51] Nathan Michael, Jonathan Fink, and Vijay Kumar. Cooperative manipulation and transportation with aerial robots. In $A u$ tonomous Robots - AROBOTS, volume 30, pages 73-86, 12011.

[52] Nathan Michael, Soonkyum Kim, Jonathan Fink, and Vijay Kumar. Kinematics and statics of cooperative multi-robot aerial manipulation with cables. In International Design Engineering Technical Conferences and Computers and Information in Engineering Conference, volume 7, pages 83-91, 012009.

[53] Volodymyr Mnih, Koray Kavukcuoglu, David Silver, Andrei A Rusu, Joel Veness, Marc G Bellemare, Alex Graves, Martin Riedmiller, Andreas K Fidjeland, Georg Ostrovski, et al. Human- 
level control through deep reinforcement learning. Nature, 518(7540):529, 2015.

[54] Multirotor. Multirotor g4 skycrane.

https://www.multirotor.net/en/products/skycrane.

[55] Vinod Nair and Geoffrey E. Hinton. Rectified linear units improve restricted boltzmann machines. In Proceedings of the 27 th International Conference on International Conference on Machine Learning, ICML'10, pages 807-814, USA, 2010. Omnipress.

[56] NASA. Scarab, nasa's newest lunar exploration rover.

https:/ / nasaeclips.arc.nasa.gov/video/realworld/archive-realworld-scarab-nasas-newest-lunar-exploration-rover.

[57] P. Neto, J. N. Pires, and A. P. Moreira. 3-d position estimation from inertial sensing: Minimizing the error from the process of double integration of accelerations. In IECON 2013 - 39th Annual Conference of the IEEE Industrial Electronics Society, pages 4026-4031, Nov 2013.

[58] R. Oung, F. Bourgault, M. Donovan, and R. D'Andrea. The distributed flight array. In 2010 IEEE International Conference on Robotics and Automation, pages 601-607, May 2010.

[59] Pedro Roque. Heterogeneous collaborative aerial manipulation. Master's thesis, KTH, Automatic Control, 2017.

[60] ROS. Robot operating system. http:/ / www.ros.org.

[61] Nicholas Roy, Paul Newman, and Siddhartha Srinivasa. Reducing Conservativeness in Safety Guarantees by Learning Disturbances Online: Iterated Guaranteed Safe Online Learning, pages 504-. MIT Press, 2013.

[62] Mouayad Sahib and Bestoun Ahmed. A new multi-objective performance criterion used in pid tuning optimization algorithms. In Journal of Advanced Research, volume 115, 042015.

[63] David Saldaña, Bruno Gabrich, Guanrui Li, Mark Yim, and Vijay Kumar. Modquad: The flying modular structure that selfassembles in midair. In ICRA'2018 - IEEE International Conference on Robotics and Automation, 2018. 
[64] David Saldana, Bruno Gabrich, Mickey Whitzer, Amanda Prorok, Mario F.M. Campos, Mark Yim, and Vijay Kumar. A decentralized algorithm for assembling structures with modular robots. In 2017 IEEE/RSJ International Conference on Intelligent Robots and Systems (IROS), Vancouver, Canada, 2017.

[65] Nordic Semiconductor. ARM-based 32-bit MCU, 256 KB Flash, $C A N, 12$ timers, ADC, DAC and comm. interfaces, $1.8 \mathrm{~V}$. November 2014. Rev. 2.

[66] Nordic Semiconductor. ARM Cortex-M4 32b MCU+FPU, 210DMIPS, up to $1 M B$ Flash/192+4KB RAM, USB OTG HS/FS, Ethernet, 17 TIMs, 3 ADCs, 15 comm. interfaces and camera. June 2013. Rev. 4.

[67] K.J. Åström and Tore Hägglund. Pid controllers : theory, design, and tuning / karl j. astrom and tore hagglund. 042018.

[68] Giri Prashanth Subramanian. Nonlinear control strategies for quadrotors and cubesats. Master's thesis, University of Illinois at Urbana-Champaign, Aerospace Engineering, 2015.

[69] TechnoSys. Skywalker x8 uav.

http://www.technosysind.com/index.php/skywalker-x8-uav/.

[70] Johannes Traa. Least-squares intersection of lines. UIUC, 2013.

[71] ASV unmanned marine systems. C-enduro.

http:/ / www.unmannedsystemstechnology.com/company/autonomoussurface-vehicles-ltd/c-enduro-solar-unmanned-surface-vehicle/.

[72] ASV unmanned marine systems. C-stat station keeping buoy. http:/ / www.unmannedsystemstechnology.com/company/autonomoussurface-vehicles-ltd/c-stat-station-keeping-buoy/.

[73] VICON. Vicon motion capture system. https: / /www.vicon.com.

[74] Christopher J. C. H. Watkins and Peter Dayan. Q-learning. Machine Learning, 8(3):279-292, May 1992.

[75] Shiqian Wu and Meng Joo Er. Dynamic fuzzy neural networks-a novel approach to function approximation. IEEE Transactions on 
Systems, Man, and Cybernetics, Part B (Cybernetics), 30(2):358-364, Apr 2000.

[76] Russell B. Wynn, Veerle A.I. Huvenne, Timothy P. Le Bas, Bramley J. Murton, Douglas P. Connelly, Brian J. Bett, Henry A. Ruhl, Kirsty J. Morris, Jeffrey Peakall, Daniel R. Parsons, Esther J. Sumner, Stephen E. Darby, Robert M. Dorrell, and James E. Hunt. Autonomous underwater vehicles (auvs): Their past, present and future contributions to the advancement of marine geoscience. $M a$ rine Geology, 352:451 - 468, 2014. 50th Anniversary Special Issue.

[77] Zarita Zainuddin. Function approximation using artificial neural networks. 2007.

[78] G. P. Zhang. Neural networks for classification: a survey. IEEE Transactions on Systems, Man, and Cybernetics, Part C (Applications and Reviews), 30(4):451-462, Nov 2000.

[79] J. G. Ziegler and N. B. Nichols. Optimum Settings for Automatic Controllers. Transactions of ASME, 64:759-768, 1942. 
TRITA TRITA-EECS-EX-2018:484

ISSN 1653-5146

www.kth.se 